\title{
Ion and mixed conducting oxides as catalysts
}

\section{P.J. Gellings and H.J.M. Bouwmeester}

Laboratory for Inorganic Chemistry, Materials Science and Catalysis, Department of Chemical Technology, University of Twente, P.O. Box 217, NL-7500 AE Enschede, the Netherlands.

\section{ABSTRACT}

This paper gives a survey of the catalytic properties of solid oxides which display oxygen ion or mixed (i.e. ionic + electronic) conductivity. Particular consideration is given to the oxidation-reduction reactions of gas phase components, but attention is also devoted to oxygen exchange between gas and oxide. An attempt has been made to relate and explain the observed phenomena such as catalytic activity and selectivity in terms of the electrical conducting properties of the oxides, which depend on their crystal and defect structures.

In a number of cases possible applications of these materials in (electro)catalytic reactors, sensors, fuel cells, oxygen pumps, etc. are indicated.

\section{INTRODUCTION}

This paper presents a review of the catalytic properties of oxides with ion or mixed, i.e. ion + electronic, conductivity. Of course in principle nearly all ion conducting oxides are also semiconductors in the sense that besides ionic defects, electronic defects are always present. However, in this paper the term mixed conduction is only used when the ionic transference number introduced in Section 3 is significantly lower than 1; otherwise the term ion conductor (or solid electrolyte) is used. Some of the systems discussed in this review exhibit high oxygen ion conduction combined with negligible electronic conduction and are used as solid electrolytes. It should be noted that this paper is mainly limited to those oxides which show oxygen ion conduction, so that, e.g., oxides with proton conduction (see, e.g. Ref. 1) are only briefly considered. As far as the present authors are aware no review on the specific topic of the present paper has appeared previously.

After giving the general background of this review, the defect chemistry of these oxides is described. The types of defects commonly found are considered, together with the most important defect equilibria. Attention is then paid to the connection between 
the properties of interest here and the nonstoichiometry and defect structure of these compounds. Following this a survey is given of the types of oxides showing mixed or ion conductivity. Their structures and some general properties are then discussed.

Subsequently surveys of important ion conducting compounds and of mixed conducting compounds, with some characteristic properties, are given in separate sections. This is followed by a short discussion of the surface chemistry of oxidic materials and of adsorption on the oxides. Oxygen exchange processes, oxygen permeation and catalytic reactions are then considered, together with a more detailed discussion of their mechanisms. As far as possible the connection between the conducting properties and the defect structures of the oxides under consideration is indicated and discussed. In a number of cases possible applications of mixed conducting oxides are considered. A short discussion about the interconnection of different phenomena discussed in this review is followed by some general conclusions. 


\section{GENERAL BACKGROUND}

Because the oxides under consideration show interesting properties, both as catalysts that may be used in practice and as model systems in the study of catalytic processes it was deemed to be useful to devote attention to this subject. Literature data indicate that many ion conducting oxides have catalytic properties which are of importance for applications as solid electrolytes in electrocatalytic reactors, fuel cells, sensors or oxygen pumps $[2,3,4]$ or as catalysts in catalytic reactors [5, 6].

It has been pointed out that in the electrochemical applications the electrode material, which acts as current collector, must be sufficiently catalytically active for most of these applications. In this connection we refer the reader to a review on electrocatalysis using cells with solid electrolytes by Gellings et al. [7]. Vayenas [8] has also recently published a review which concentrates on the behaviour of metal electrodes and their catalytic behaviour in solid oxide fuel cells.

On the other hand a catalytic contribution of the electrolyte itself has also been demonstrated in several cases (e.g. Ref. 9 and the references in Section 12.10). Attention is mainly focused on those systems in which the oxygen ion conductor itself takes part in the catalytic reaction or where oxidic electrode materials are used in solid electrolyte electrochemical cells.

Thomas [10], in discussing the possibility of obtaining a better control of the performance of catalysis by solids, mentions as one important group the complex oxides. The facile loss and transport of structural oxygen in these compounds are suggested as being of particular importance.

Several reviews on the catalytic properties of oxidic materials have been published relatively recently. In particular the papers by Voorhoeve [11] and Sleight [12] must be mentioned in this respect. Claudel [13] presents a general discussion of adsorption and catalysis on ionic solids with some applications to ion conductors such as uranium doped thoria and nonstoichiometric ceria. More recently Cimino and Carrà [14] published a review on chemisorption and catalysis on metal oxides in which they also attempted to rationalize adsorption and catalytic phenomena and in which attention was also paid to the influence of the semiconducting properties of the oxides. Sokolovskii [15] discussed the principles of oxidative catalysis on solid oxides in general in a recent review, emphasizing the contributions of Russian investigators to the knowledge in this field.

A survey of the surface chemistry and catalysis of transition metal oxides has been published by Kung [16] without, however, any reference to the defect structures or possible ion conducting properties of the oxides considered and of their influence on the catalytic properties.

Reviews which discuss bulk and/or surface properties of ion conducting materials 
of the type considered in this paper have been published by Worrell [17], Dell and Hooper [18], Bergmann and Tannenberger [19] and Burggraaf et al. [20]. The last authors in particular discuss these oxidic materials from different points of view, to which reference is made at other points in the present review. A recent survey of the background of solid state electrochemistry as applied to ion and mixed conductors has been published by Kudo and Fueki [21] and this gives an excellent introduction to this field.

Oxide catalysts which lose or take up oxygen during a catalytic reaction may show a drastic change in their conducting and catalytic properties [22]. In those cases where the parent oxide is (practically) stoichiometric and an insulator they become mixed valence compounds, which usually means that they become n- or p-type semiconductors.

A special issue of this journal [23] has been devoted to one specific class of materials some of which are ion or mixed conductors - the perovskites - and the separate papers in that issue are referred to later.

The ion or mixed conducting properties of an oxide will certainly influence its catalytic behaviour, but in many cases it is difficult to unequivocally determine this contribution among the many other effects, because the catalytic properties of any material are determined by a subtle interplay of a large number of factors (see also Refs. 24, 25):

- $\quad$ crystal and surface structure of the catalyst [26];

- molecular structure of reactants;

- thermodynamic stability of reactants and catalyst;

- $\quad$ acid-base properties of the oxide surface [27];

- $\quad$ influence of ionic/covalent bonding effects [28, 29];

- defect properties of the catalyst, both in connection with conduction properties and with surface defects;

- $\quad$ electronic and semiconducting properties and band structure [13, 30];

- $\quad$ coexistence of different types of ions or structures;

- $\quad$ crystal field effects in transition metal ions;

- types of adsorbed reactants such as oxygen (see e.g. Ref. 31).

Furthermore other factors also influence the measured catalytic activity besides surface and bulk properties of the catalyst. As shown by Barnard et al. [32] the effect of the preparation of a catalyst may have a very great influence on surface area and thus on the experimental behaviour, which is not always taken into account in the expression and comparison of reaction rates per gram of catalyst and not per unit surface area.

Only a limited number of systematic investigations have been performed of the catalytic properties and their correlation with the conducting properties of known ion or mixed conductors, so that it is difficult to enable valid correlations. Many of these investigations remain isolated and further research is certainly needed to bridge the gaps in the present knowledge of this field. 


\section{DEFECT CHEMISTRY OF OXIDES}

\subsection{Introduction}

Within the limits of this review only a brief introduction into the main points of defect chemistry can be given. For more extensive treatments, reference must be made to the literature $[33,34,35,36]$.

\subsection{Types of defects}

Ion conductivity or diffusion in oxides can only take place because of the presence of imperfections or defects in the lattice. A finite concentration of defects will be present at all temperatures above $0 \mathrm{~K}$ arising from the entropy contribution to the Gibbs free energy as a consequence of the disorder introduced by the presence of the defects.

From an elementary thermodynamic consideration of the problem it follows that the mole fraction of defects, assuming that this is not too big, is given by:

$$
\mathrm{x} \propto \exp \left(-\frac{\Delta \mathbf{H}_{\mathrm{f}, \mathrm{def}}}{\mathrm{RT}}\right)
$$

where $\Delta \mathrm{H}_{\mathrm{f}, \mathrm{def}}$ is the formation enthalpy of the defects considered. Because these formation enthalpies usually differ greatly for different defects it is often a good approximation to consider that only one type of defect is present: the so called majority defect.

The defects under consideration here may, for example, be vacant lattice sites, ions placed at normally unoccupied sites (so-called interstitial ions), foreign ions present as impurity or dopant and ions with charges different from those expected from the overall stoichiometry. Electron defects may arise in the form of ions present with charges deviating from the normal lattice ions, as well as a consequence of the transition of electrons from normally filled energy levels, usually the valence band, to normally empty levels, the conduction band. In those cases where an electron is missing, i.e. when there is an electron deficiency, this is usually called a hole (or electron hole). Though actually ions (or electrons) move through the lattice, in the case of vacancies and electron holes, it is more convenient to consider the point defects to be the moving entities.

In ionic lattices, in the absence of macroscopic electric fields, it is required that they are electrically neutral everywhere. This overall charge neutrality requires that a charged defect must be compensated by a number of defects, together having a complementary charge of opposite sign; thus these charged defects are always present as a combination of two types of defects. The charges of defects and of the regular lattice particles are only important with respect to the neutral, unperturbed (ideal) lattice. In the following discussion the charges of all point defects are all given relative to the neutral unperturbed lattice. Thus only effective charges are considered and these are indicated by a dot $(\mathcal{C})$ for a positive excess charge, and by a prime ( ${ }^{\prime}$ ) for a negative excess charge. The notation used for defects is from Kröger and Vink [37] and is given in Table 1. 
Only fully ionized defects are indicated in this table. For example, considering anion vacancies we could, besides doubly ionized anion vacancies: $V_{X}$ " , also have singly ionized or uncharged anion vacancies: $\mathrm{V}_{\mathrm{X}}{ }^{\cdot}$ or $\mathrm{V}_{\mathrm{X}}{ }^{\mathrm{X}}$, respectively.

\section{Table 1}

Kröger-Vink notation for point defects in crystals

\begin{tabular}{|c|c|c|}
\hline Type of defect & Symbol & Remarks \\
\hline vacant $M$ site & $\mathrm{V}_{\mathbf{M}^{\prime \prime}}$ & $\begin{array}{l}\text { Divalent ions are chosen as example } \\
\text { with } \mathrm{MX} \text { as compound formula }\end{array}$ \\
\hline vacant $X$ site & $v_{X}{ }^{*}$ & $\mathrm{M}^{2+}, \mathrm{X}^{2-}$ : cation and anion \\
\hline ion on lattice site & $\mathrm{M}_{\mathrm{M}}^{\mathrm{x}}, \mathrm{X}_{\mathrm{X}}^{\mathrm{x}}$ & $x$ : uncharged \\
\hline L on $M$ site & $\mathbf{L}_{\mathbf{M}}^{\prime}$ & $\mathrm{L}^{+}$dopant ion \\
\hline $\mathrm{N}$ on $\mathrm{M}$ site & $\mathrm{N}_{\mathrm{M}} \cdot$ & $\mathrm{N}^{3+}$ dopant ion \\
\hline free electron & $e^{\prime}$ & \\
\hline free (electron) hole & $h^{*}$ & \\
\hline interstitial $M$ ion & $\mathbf{M}_{\mathrm{i}}^{* *}$ & $\because$ : effective positive charge \\
\hline interstitial $\mathrm{X}$ ion & $x_{i}^{\prime}$ & : effective negative charge \\
\hline
\end{tabular}

Two common types of disorder in ionic solids are Schottky and Frenkel defects. At the stoichiometric composition the presence of Schottky defects involves equivalent amounts of cation and anion vacancies. In the Frenkel defect structure defects are limited to either the cations or the anions of which both a vacancy and an interstitial ion are present. An anion Frenkel defect is sometimes called an anti-Frenkel defect. Ionic defects which are present due to the thermodynamic equilibrium of the lattice are called intrinsic defects.

Foreign ions in a lattice (substitutional ions or foreign ions present on interstitial sites) are one type of extrinsic defect. When aliovalent ions (impurities or dopes) are present the concentrations of defects of lattice ions will also be changed and they may become so large that they can be considered to be a kind of extrinsic defect too, in particular when they form minority defects in the absence of foreign ions. For example, dissolution of $\mathrm{CaO}$ in the fluorite phase of zirconia $\left(\mathrm{ZrO}_{2}\right)$ leads to $\mathrm{Ca}^{2+}$-ions occupying $\mathrm{Zr}^{4+}$-sites, and an effectively positive charged oxygen vacancy is created for each $\mathrm{Ca}^{2+}$-ion present to preserve electroneutrality. The defect reaction can be written as: 
$\mathrm{CaO} \rightarrow \mathrm{Ca}_{\mathrm{Z}_{\mathrm{r}}^{\prime \prime}}+\mathrm{O}_{\mathrm{o}}^{\mathrm{x}}+\mathrm{V}_{\mathrm{O}}^{*}$

with the electroneutrality condition or charge balance:

$\left[\mathrm{Ca}_{\mathbf{Z x}^{\prime \prime}}\right]=\left[\mathrm{v}_{\mathbf{0}}^{\infty}\right]$

where the symbol of a defect enclosed in brackets denotes its mole fraction. Consequently, in this situation the mole fraction of ionic defects is fixed by the amount of dopant ions present in the oxide.

Nonstoichiometry occurs when there is an excess of one type of defect relative to that at the stoichiometric composition. Since the ratio of cation to anion lattice sites is the same whether a compound is stoichiometric or nonstoichiometric, this means that complementary electronic defects must then be present to preserve electroneutrality.

The extent of electronic conductivity for a non-degenerate semiconductor is determined by the value of the electronic band gap, $\mathbf{E}_{\mathbf{g}}$. The intrinsic ionization across the band gap can be expressed by:

$$
\begin{aligned}
& e^{\prime}+h^{\bullet}=0 \\
& n \times p=N_{c} \times N_{v} \times \exp \left(-\frac{E_{g}}{k T}\right)
\end{aligned}
$$

where $n$ and $p$ are the numbers of electrons and of electron holes, $N_{c}$ and $N_{v}$ are the effective densities of states in the conduction and valence band, respectively, and $\mathbf{E}_{\mathrm{g}}$ is the band gap energy. In ionic solids the mobility of electrons is largely determined by the interaction with optical phonons. The electron induces a local polarization in the surrounding lattice which is dragged along with it upon migration. The combination of electron and its strain field is referred to as a polaron. It is common to speak of large and small polarons. In the former case the coupling with the lattice is small, the electron moves in a band with an apparent, small increase in electron mass. The electrons associated with small polaron are self-trapped at a given lattice site and can only migrate to an adjacent lattice by thermally activated hopping. The reported mobilities at elevated temperatures are $10^{-4}-10^{-2} \mathrm{~cm}^{2} / \mathrm{V} . \mathrm{s}, 2-3$ orders smaller than in large polaron conductors [35]. In the case of hopping type semiconductivity $N_{c}$ and $N_{v}$ represent the number of ions which have lost or gained one or more electrons.

Instead of $n$ and $p$ the concentrations [ $\left.e^{\prime}\right]$ and $\left[h^{\circ}\right]$ are often used where $\mathbf{n}=\mathbf{N}_{\mathrm{Av}} \times\left[\mathrm{e}^{\prime}\right]$ and $\mathrm{p}=\mathbf{N}_{\mathrm{Av}} \times\left[\mathrm{h}^{\prime}\right]$. In that case the equilibrium condition is usually written in the form:

$\mathrm{K}_{\mathrm{el}}=\left[\mathrm{e}^{\prime}\right] \times\left[\mathrm{h}^{ }\right]$

The total conductivity consisting of the contributions from both ionic and 
electronic defects is given by:

$$
\begin{aligned}
\sigma_{\text {total }} & =\sigma_{\text {ion }}+\sigma_{\mathrm{c}}+\sigma_{\mathrm{h}} \\
& =\sum \mathrm{c}_{\mathrm{i}}\left|\mathrm{z}_{\mathrm{i}}\right| \mathrm{e}_{0} \mu_{\mathrm{i}}+\mathrm{n} \mathrm{e}_{0} \mu_{\mathrm{e}}+\mathrm{p} \mathrm{e}_{0} \mu_{\mathrm{h}}
\end{aligned}
$$

where $c_{i}$ is the concentration of 'free' non-interacting ionic defects of type $i$ with charge number $z_{i}, e_{0}$ is the elementary charge, $\mu$ the electrical mobility and the subscripts $i, e$ and $\mathrm{h}$ denote ions, electrons and electron holes, respectively. When only one type of ionic defect (in our case either $\mathrm{O}^{\prime \prime}$ or $\mathrm{V}_{\mathrm{O}}{ }^{\text {") }}$ ) contributes to the conduction we write $\sigma_{\text {ion }}{ }^{\wedge} \mathrm{Xxr}$ the ionic contribution to the total conductivity. The mobilities of electronic defects are in general a factor of 1000 or more larger than those of ionic defects. This means that, in order to have a purely ionic conducting solid electrolyte, a minimum band gap energy is required and $\mathrm{E}_{\mathrm{g}}$ must usually be larger than approximately $3 \mathrm{eV}$.

Between the different defects there exists a coulombic interaction due to their charges. In the case of oppositely charged defects this may lead to the formation of defect associates, corresponding to a decrease in energy. This formation of defect associates usually causes a decrease of the total conductivity [38].

A useful concept in describing solids in which there is more than one type of charge carrier is the transport (or transference) number $t_{k}$ for any carrier $k$, defined by: $t_{k}=\sigma_{k} / \sigma_{\text {total }}$

In cases where ionic and electronic conduction occur together, i.e. in the case of mixed conduction:

$\sigma_{\text {total }}=\sigma_{\text {ion }}+\sigma_{\text {el }}=\sigma_{\text {total }} \times\left(t_{\text {ion }}+t_{\text {el }}\right)$

where $t_{i o n}$ and $t_{e l}$ represent the transport numbers of ionic and electronic defects, respectively. It may be noted that $t_{\text {ion }}+t_{e l}=1$. Furthermore $t_{e l}=t_{e}+t_{h}$.

\subsection{Defect equilibria}

The extent of nonstoichiometry and the defect concentrations in solids are functions of the temperature and the partial pressure of their chemical components (for an extensive treatment of defect equilibria and their consequences, see Ref. 36). Under certain conditions this may lead to an increase in electronic conductivity (either n-type or p-type) so that a solid electrolyte becomes a mixed conductor.

As an example we consider an oxide $\mathrm{MO}_{2}$ with Frenkel defects in the anion sublattice. As the partial pressure of the metal component is negligible compared with that of oxygen under most experimental conditions, nonstoichiometry is correspondingly a result of the interaction of the oxide with the oxygen in the surrounding gas atmosphere.

The Frenkel defect equilibrium for the oxygen ions can be written as:

for fully ionized defects, as is usually observed in oxides. The thermal equilibrium between electrons in the conduction band and electron holes in the valence band is rep- 
$\mathrm{O}_{\mathrm{O}}^{\mathrm{x}}=\mathrm{O}_{\mathrm{i}}^{\prime \prime}+\mathrm{V}_{\mathrm{O}}^{\infty}$

$\mathbf{K}_{\mathrm{F}}=\left[\mathbf{O}_{\mathbf{i}}{ }^{m}\right] \times\left[\mathbf{V}_{\mathbf{O}}^{\infty}\right]$

resented by Eqns. (4). Taking into account the presence of electrons and electron holes the electroneutrality condition then reads:

$\left[\mathrm{h}^{*}\right]+2\left[\mathrm{~V}_{\mathrm{O}}^{\infty}\right]=\left[\mathrm{e}^{\prime}\right]+2\left[\mathrm{O}_{\mathrm{i}}^{\prime \prime}\right]$

If ionic defects predominate, the concentrations of oxygen interstitials $O_{i}$ " and oxygen vacancies $\mathrm{V}_{\mathrm{O}}{ }^{\circ}$ (because $\left[\mathrm{V}_{\mathrm{O}^{*}}\right]$ " $\left[\mathrm{h}^{\circ}\right]$ and $\left[\mathrm{O}_{\mathrm{i}}{ }^{\prime \prime}\right]$ » $\left[\mathrm{e}^{\prime}\right]$ ) are equal and independent of oxygen pressure.

As the oxygen pressure is increased, oxygen is being increasingly incorporated into the lattice, the corresponding defect equilibrium being:

$$
\begin{aligned}
& 1 / 2 O_{2}=O_{i^{\prime \prime}}+2 h^{*} \\
& K_{o x} \times \mathrm{p}_{O_{2}}^{1 / 2}=\left[O_{i^{\prime \prime}}\right] \times\left[h^{\circ}\right]^{2}
\end{aligned}
$$

This type of equilibrium, which involves p-type semiconductivity, is only possible if cations are present which have the capability of increasing their valence. As the oxygen pressure is decreased, oxygen is increasingly being removed from the lattice, the corresponding defect equilibrium being:

$$
\begin{aligned}
& \mathrm{O}_{\mathrm{O}}^{\mathrm{x}}=1 / 2 \mathrm{O}_{2}(\mathrm{~g})+\mathrm{V}_{\mathrm{O}}^{\infty}+2 \mathrm{e}^{\prime} \\
& {\left[\mathrm{V}_{\mathrm{O}}^{\infty}\right] \times\left[\mathrm{e}^{\prime}\right]^{2}=\mathrm{K}_{\mathrm{red}} \times \mathrm{p}_{\mathrm{O}_{2}}^{-1 / 2}}
\end{aligned}
$$

noting that $\left[\mathrm{O}_{\mathrm{O}} \mathrm{x}\right] \approx 1$.

This type of equilibrium, which involves n-type semiconductivity, is only possible if cations are present which have the capability of decreasing their valence. In this case of predominantly ionic defects, the concentrations of oxygen interstitials and of oxygen vacancies are constant, as stated above. We then obtain from Eqns. (11) and (12):

$$
\left[\mathrm{h}^{*}\right] \propto \mathrm{p}_{\mathrm{O}_{2}}^{1 / 4}
$$

or:

$$
\left[\mathrm{e}^{\prime}\right] \propto \mathrm{p}_{\mathrm{O}_{2}}^{-1 / 4}
$$

The above shows that both the direction and the degree of nonstoichiometry depend on the availability of an adjacent oxidation state of the constituent ions, usually the cation, regardless of the nature of the electronic defects. An oxidizable or reducible cation favours an excess or deficiency of oxygen, respectively. As a corroboration of this behaviour, oxides such as $\mathrm{MgO}$ and $\mathrm{Al}_{2} \mathrm{O}_{3}$ in which the cation has only a single stable oxidation state are line compounds, indicating that the metal to oxygen ratio cannot vary. 
The ease of valency changes can be correlated with the ionization energies of donor and acceptor levels, which correspond with the energies of charged defects. These levels lie within the forbidden band between the filled valence and the empty conduction band. The valence band usually consists mainly of filled oxygen $2 p$ orbitals. For oxides of transition metals in their highest valence state the conduction band in general consists of the empty $d$ levels of the metal ions. Schematically this band structure is shown for some cases in Fig. 1.

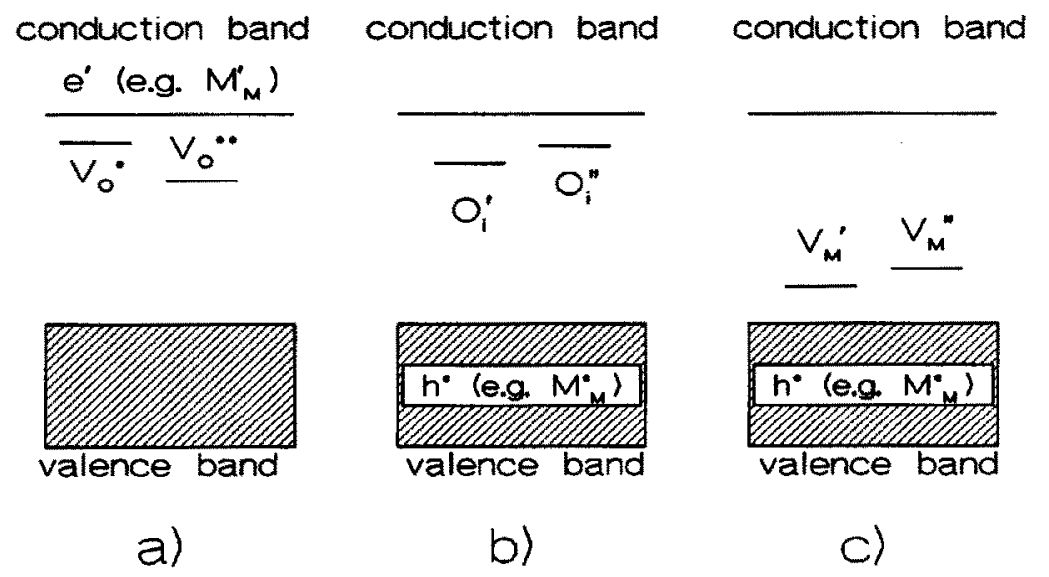

Fig. 1. Schematic band level schemes for oxide: (a) with oxygen vacancies, (b) with oxygen interstitials, (c) with cation vacancies.

When only lower oxidation states are available, as in $\mathrm{ZrO}_{2}$, the donor levels, which can be, for example, oxygen vacancies as shown in Fig. 1.(a), lie close to the bottom of the conduction band, reflecting the ease of charge donation to the lowest unfilled $\mathrm{d}$ band of $\mathrm{Zr}$. As a consequence this type of compound is an n-type semiconductor and this is shown in Fig. 1.(a). Reduction increases the conductivity and this type of compound is called a reduction type semiconductor. Oxidation would involve the creation of electron holes, e.g. in the form of $\mathrm{Zr}^{5+}$ or $\mathrm{O}^{-}$, which is energetically very unfavorable because the corresponding ionization energies are very high. The corresponding acceptor levels are, for example, oxygen interstitials as shown in Fig. 1.(b). In principle this could occur at very high oxygen partial pressures.

When the cation in an oxide, such as in compounds like NiO, can assume a higher valency, metal ion vacancies can be formed. These act as acceptors because they lie close to the valence band, as illustrated in Fig. 1.(c). These compounds are called oxidation type semiconductors and show p-type semiconductivity. For a more thorough treatment of this subject we refer the reader to the literature, e.g. Refs. 33, 39. 
An additional factor in determining the extent of nonstoichiometry is the energy needed to form structural disorder. For example, the ternary oxide $\mathrm{CaTiO}_{3}$ can easily accomodate an excess of $\mathrm{Ca}$ because the $\mathrm{Ca}^{2+}$ ion is small enough to substitute for a $\mathrm{Ti}^{4+}$ ion, which resides on octahedral sites of the perovskite structure, which is described in Section 4.1.

In the foregoing only single defects have been considered, but, as has been mentioned earlier, it will be clear that there will also be interactions between different defects, mainly due to their charges. At low defect concentrations these can usually be neglected, but at higher concentrations this is not possible any longer and defect associates are formed. This was shown, for example, by Van Roosmalen and Cordfunke [40] for perovskites of the type $\mathrm{LaMO}_{3-\delta}(\mathrm{M}=\mathrm{Mn}, \mathrm{Fe}, \mathrm{Co})$ for relatively high values of $\delta$. In this case a defect cluster, consisting of two $\mathrm{M}^{2+}$ ions bound to one oxygen vacancy is necessary to describe the defect properties.

At a large excess or deficit of oxygen new limiting conditions are deduced from the electroneutrality condition and consequently power dependencies different from those of Eqns. (13) and (14) are calculated from the mass action expressions.

For example at very low oxygen partial pressures Eqns. (11) and (12) show that $\left[\mathrm{V}_{\mathrm{O}}{ }^{\circ}\right]$ and $\left[\mathrm{e}^{\prime}\right]$ are then expected to be large compared with $\left[\mathrm{O}_{\mathrm{i}}{ }^{*}\right]$ and $\left[\mathrm{h}^{\circ}\right]$. The electroneutrality condition, equation (10), then becomes to a good approximation:

$$
2\left[\mathrm{~V}_{\mathrm{O}}^{\infty}\right] \approx\left[\mathrm{e}^{\prime}\right]
$$

and this gives:

$\left[e^{\prime}\right] \propto \mathrm{P}_{\mathrm{O}_{2}}^{-\frac{1}{6}}$

These phenomena are clearly illustrated in so-called Brouwer or Kröger-Vink diagrams [33, 41], where the defect concentrations are shown as a function of the activity of one component, usually a gaseous nonmetal, in the case of oxides $\mathrm{O}_{2}$. A (schematic) equilibrium defect diagram of this type is shown in Fig. 2.(a) for the pure (fluorite) oxide $\mathrm{MO}_{2}$. The partial conductivities are plotted in Fig. 2.(b). As can be seen the electrolytic domain is separated from pure semiconducting regions by mixed conducting regions. Since the mobilities of the electronic charge carriers generally tend to be much larger than those of ionic defects, the ionic or electrolytic domain will in fact be smaller than indicated in Fig. 2.(b), where for clarity approximately equal mobilities of the different defect species are used.

For oxides like $\mathrm{ZrO}_{2}$ or $\mathrm{TiO}_{2}$, where a higher valence state is not available (reduction type semiconductors) the atmospheric oxygen pressure is expected to lie to the left of the center of this figure, reflecting the difficulty of creating electron holes in these 
compounds. On the other hand for structurally similar oxides like $\mathrm{VO}_{2}$ or $\mathrm{NbO}_{2}$, where a higher oxidation state of the metal is available (oxidation type semiconductors) the atmospheric oxygen pressure is expected to lie more to the extreme right of this figure.

\subsection{Properties}

The majority defects in pure $\mathrm{ZrO}_{2}$ are oxygen vacancies $\mathrm{V}_{\mathrm{O}}{ }^{\circ}$ and electrons $e^{\circ}$, both at very low concentrations. The addition of lower valent oxides, such as $\mathrm{CaO}$ or $\mathrm{Y}_{2} \mathrm{O}_{3}$, gives the stabilized zirconias. This increases the oxygen vacancy concentration and thus leads to a much larger ion conductivity. At the same time the relative electronic contribution to the conductivity, which is already small, decreases even more. The electroneutrality condition becomes; to a good approximation:

$2\left[\mathrm{~V}_{\mathrm{O}}^{\infty}\right]=2\left[\mathrm{Ca}_{\mathrm{Zr}_{\mathrm{r}}}\right]$

or

$2\left[\mathrm{~V}_{\mathrm{O}}^{* 0}\right]=\left[\mathbf{Y}_{\mathbf{Z r}^{\prime}}\right]$

with either $\mathrm{Ca}^{2+}$ or $\mathrm{Y}^{3+}$ being the stabilizing cation and where [e $e^{\prime}$ can be neglected with respect to the concentration of the substitutional stabilizing cations. Under these circumstances the ionic conductivity becomes independent of the oxygen partial pressure and is completely determined by the dopant concentration.

The 'partial pressure window', that is the range of partial pressures in which a compound is an ionic conductor, is relatively wide for the stabilized zirconias, covering about 20 - 30 orders in magnitude around the minumum of electronic conductivity, but considerably narrower for $\mathrm{CeO}_{2}$ and $\mathrm{Bi}_{2} \mathrm{O}_{3}$ based solid electrolytes.

Rifflet and Odier et al. [42, 43] used measurements of the thermal emission of electrons in the study of point defects in refractory oxides, among others in yttria stabilized zirconia $\left(\mathrm{ZrO}_{2}-7.5 \% \mathrm{Y}_{2} \mathrm{O}_{3}\right)$. As shown by these authors one of the advantages of this method is that, at sufficiently high temperatures, the thermal emission current is directly proportional to the electron concentration even if the electrons are present as minority defects. Experimentally they observed that the thermal emission current is proportional to $\mathrm{PO}_{2}{ }^{-1 / 4}$, in agreement with equation (14).

For the fluorite oxide $\mathrm{MO}_{2}$ doped with $\mathrm{D}_{2} \mathrm{O}_{3}$ the schematic Brouwer diagram is shown in Fig. 3.(a). The partial conductivities are plotted in Fig. 3.(b) again assuming approximately equal mobilities for all defect species. 


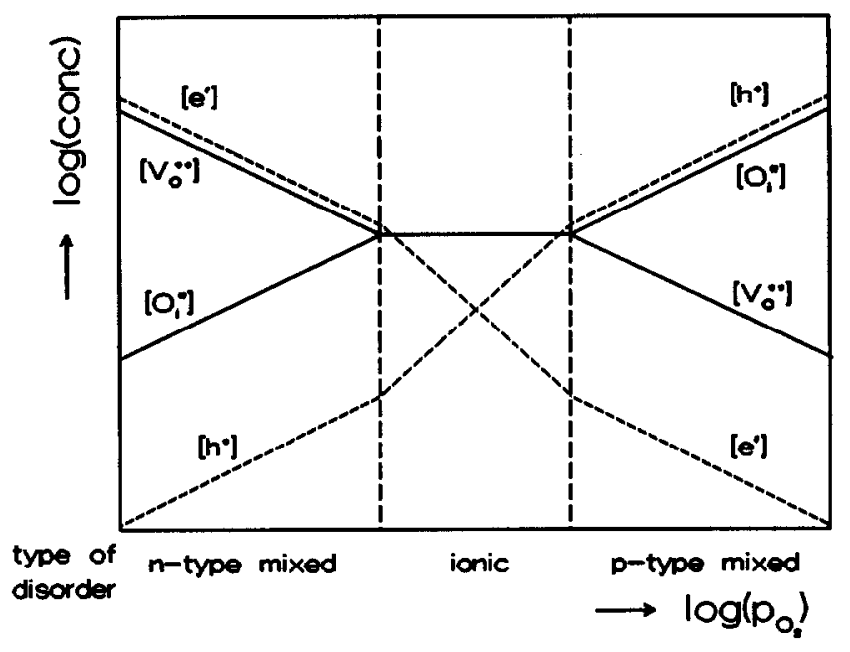

a)

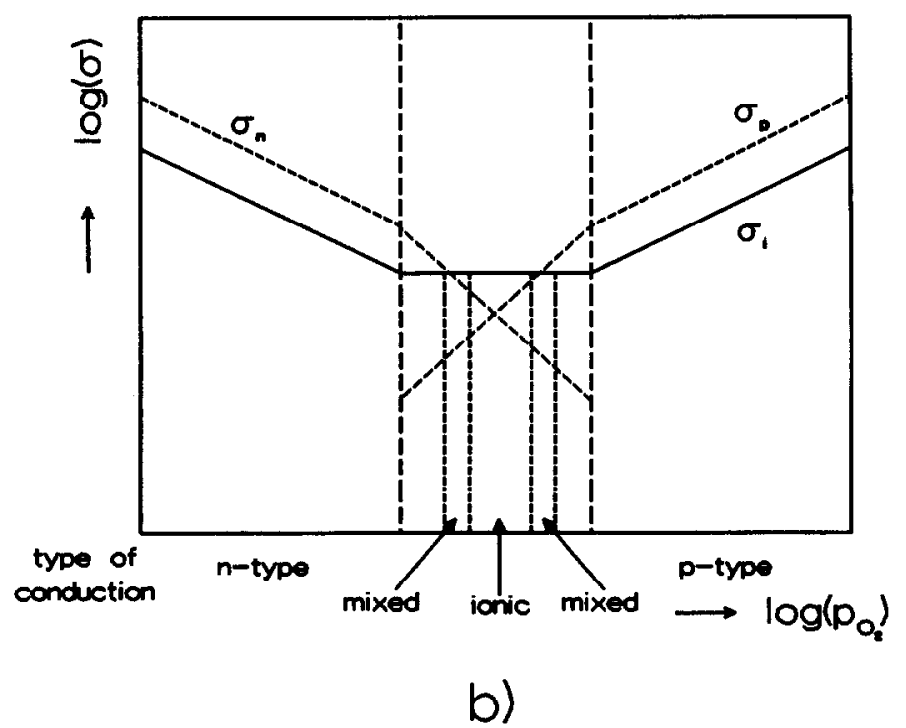

Fig. 2. (a) Brouwer diagram for pure $\mathrm{MO}_{2}$, (b) partial conductivities of defects. 


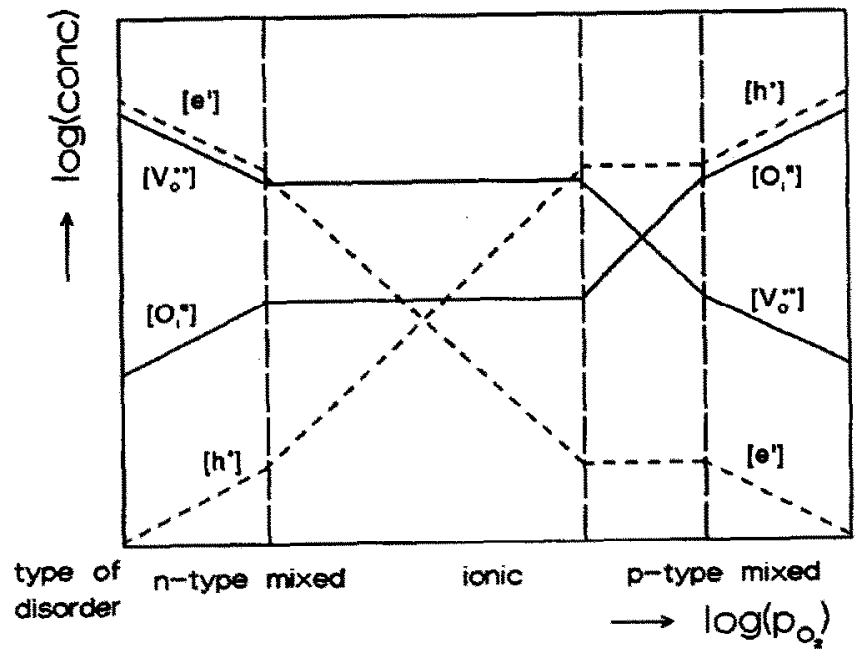

a)

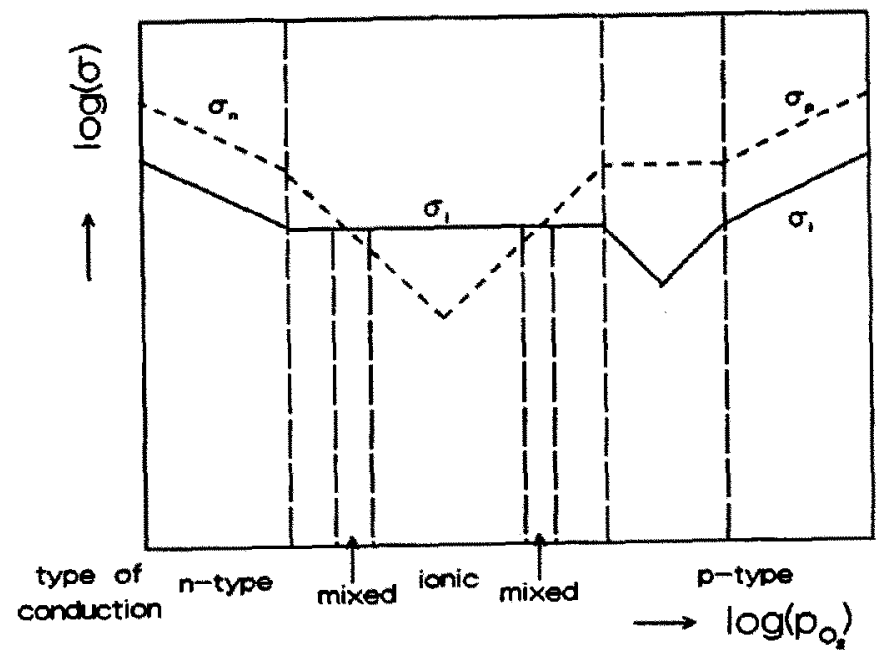

b)

Fig. 3.(a) Brouwer diagram for $\mathrm{MO}_{2}$ doped with $\mathrm{D}_{2} \mathrm{O}_{3}$, (b) partial conductivities of defects. 
In the early development of oxygen conducting fluorite-type ceramics, emphasis was in expanding the 'partial pressure window', which defines the activity range for succesful application as electrolytes in solid oxide fuel cells, oxygen sensors, pumps etc. Only recently electronic conductivity has been brought about deliberately into highly oxygen conducting matrices by introducing dopants with variable valencies, whereby electronic migration becomes possible via the polaron mechanism, mentioned in Section 3.2. Examples of technological applications incorporating oxide mixed conductors include: ceramic membranes designed to separate oxygen from air, and oxide electrodes for solid oxide fuel cells, oxygen sensors or oxygen pumps. Materials based on yttria stabilized zirconia in which either $\mathrm{Ce}, \mathrm{Cr}$ or $\mathrm{Ti}[44,45,46,47,48]$ is dissolved seem most promising. The Kröger-Vink or Brouwer diagram can be obtained as outlined above, taking into account the redox equilibrium between the two valence states of the mixed-valence cation, as has been thoroughly analyzed by Tuller [35] and Ramanarayanan et al. [49]. Since a material, in order to be useful in practice for the applications considered here, must transport oxygen ions and electrons in the appropriate range of oxygen partial pressure, the redox equilibrium must be within this 'partial pressure window'.

A convenient way to illustrate the temperature dependence of the ionic (and electrolytic) domain boundaries is shown in Fig. 4, a type of diagram first introduced by Patterson [50]. The narrowing of these boundaries at higher temperatures is due to the higher activation enthalpy of the partial electronic conductivity compared with that of the ionic conductivity. The low energy of activation of the ionic conductivity reflects the ease of ions in surmounting an energy barrier (hopping model), whereas the high activation enthalpy for the electronic conductivity corresponds with the large band gap required for good solid electrolytes, as discussed above.

\subsection{Relations between transport properties}

The possibility of diffusion in a solid is a consequence of the presence of (point) defects and this can be considered in principle as a random (Brownian) movement process of ions or defects in the lattice. This process is characterized by a diffusion coefficient. For the totally uncorrelated movement of a particle $i$ in the crystal, in the absence of concentration gradients, it is given by:

$D_{i}=\frac{1}{6} \nu r^{2}$

where $\nu$ is the jumping frequency of ions or defects in the lattice and $r$ the jumping distance. The diffusion coefficient defined in Eq. (19) is called the self diffusion coefficient and is correlated by the Einstein equation with the mobility $b_{i}$ of the particles: 


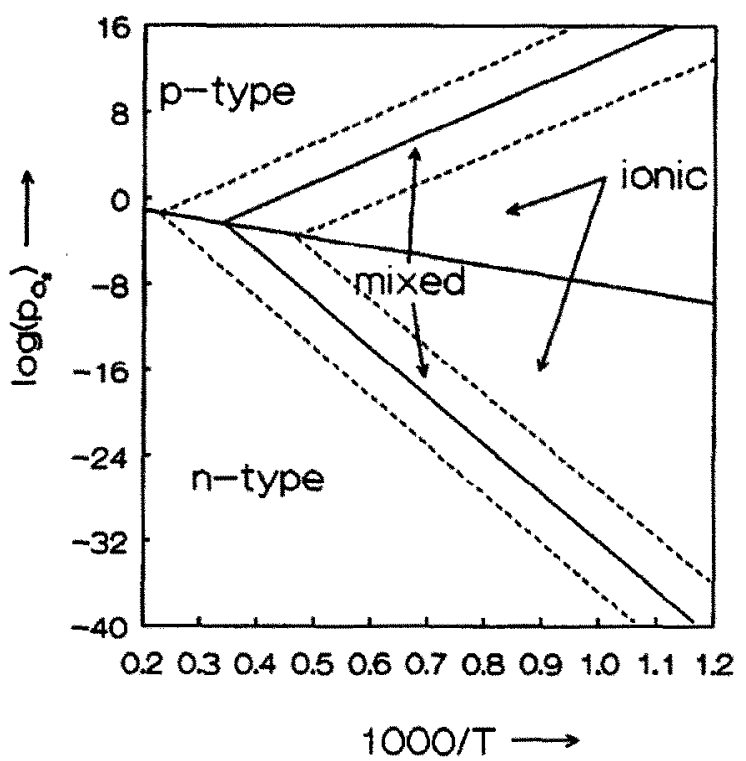

Fig. 4. Patterson diagram.

$D_{i}=k_{B} T b_{i}$

where $\mathrm{k}_{\mathrm{B}}$ is Boltzmann's constant.

In a crystal there are unequal jumping probabilities in different directions and is taken into consideration by introducing a correlation factor, $f$, which depends both on the type of diffusing entity and on the crystal structure. This then gives the tracer diffusion coefficient, indicated by $\mathrm{D}_{\mathrm{i}}^{*}$ :

$D_{i}^{*}=\frac{1}{6} \nu r^{2} f$

Often for charged defects the electrical mobility, $\mu_{\mathrm{i}}$, is used. This is defined as the mobility per unit of electric field strength. Combined with Eq. (20) leads to:

$\mu_{\mathrm{i}}=z_{\mathrm{i}} \mathrm{e}_{0} \mathrm{~b}_{\mathrm{i}} \rightarrow \mathrm{D}_{\mathrm{i}}=\frac{\mathrm{k}_{\mathrm{B}} \mathrm{T}}{\mathrm{z}_{\mathrm{i}} \mathrm{e}_{0}} \times \mu_{\mathrm{i}}=\frac{\mathrm{RT}}{\mathrm{z}_{\mathrm{i}} \mathrm{F}} \times \mu_{\mathrm{i}}$

where $z_{i}$ is the charge of the ion or defect, $e_{0}$ the elementary charge and $F$ the Faraday constant.

The macroscopically measurable transport through a solid is a function of the tracer diffusion coefficient as defined above and of the concentration of the diffusing entities. This leads to the so-called defect diffusion coefficient as a product of the tracer 
diffusion coefficient and the mole fraction of the defect involved. As the defect concentration is strongly influenced by doping and by changes in temperature these have a corresponding influence on the defect diffusion coefficient.

The contribution of the movement of a certain defect or ion in an electric field to the conductivity depends, like the defect diffusion coefficient, also on the concentration of the defect. If this has a mole fraction $x_{i}$ and the total number per mole is $n_{i}=N_{A v} \times$ we obtain for the relation between conductivity and electrochemical mobility:

$\sigma=\mathrm{n}_{\mathrm{i}} \mu_{\mathrm{i}} \mathbf{z}_{\mathrm{i}} \mathrm{e}_{\mathbf{0}}=\mathrm{x}_{\mathrm{i}} \mu_{\mathrm{i}} \mathbf{z}_{\mathrm{i}} \mathrm{F}$

Combining this with Eq. (22) the relation between the contribution of a defect to the conductivity and its diffusion coefficient is derived to be:

$\sigma=D_{i}\left(z_{i} F\right)^{2} \frac{x_{i}}{R T}$

In ionic solids electroneutrality must be retained everywhere in the solid throughout a diffusion process. This means that always at least two different types of charged defects must move simultaneously, e.g. oxygen vacancies and electrons in the same direction or oxygen interstitials and electrons in opposite directions. Due to the unequal mobilities (or defect diffusion coefficients) of the defects this leads to a local deviation from stoichiometry and hence to an electric field in the solid. This accelerates the slow moving defect, while retarding the fast moving one, until they move at identical speeds. The diffusion coefficient which describes the coupled motion of the two types of defects is called the chemical diffusion coefficient and is closely related to the defect diffusion coefficients of the moving species involved.

Within the scope of this review it is not possible to go deeper into a consideration of the different types of diffusion coefficient or into the different models for the diffusion process. For this reference must be made to the literature, e.g., to the books by Kudo and Fueki [21], Kofstad [36], Borg and Dienes [51] and the paper by Murch [52]. 


\section{OXIDES}

In this section we give a review of the different oxides that are of interest to the scope of the present paper. Their structural types are introduced, followed by a short description of some important properties and their connection with the structure.

\subsection{Structural types}

The fluorite structure is one of the most important structures in the field of ion conducting oxides. It is the structure of the high temperature form of $\mathrm{ZrO}_{2}$ and of the so-called stabilized zirconias. At room temperature $\mathrm{ZrO}_{2}$ has a deformed structure, derived from the fluorite $\left(\mathrm{CaF}_{2}\right)$ structure. Pure $\mathrm{ZrO}_{2}$, when cooled from high temperatures, undergoes several phase transitions which are accompanied by relatively large volume changes. The cubic structure is shown in Fig. 5. Due to the volume changes caused by the phase transitions sintered compacts of pure $\mathrm{ZrO}_{2}$ pulverise on cooling. By doping $\mathrm{ZrO}_{2}$ with lower valent ions such as calcium or yttrium the cubic structure is stabilized and remains viable down to room temperature, even though it is metastable at that temperature.

In the stabilized zirconias the oxygen vacancies, which are present as the ionic majority defects, are randomly distributed over the (identical) crystallographic oxygen positions. In particular, ordering effects may occur at high dopant concentrations, depending on the ratio of the radii of the $\mathrm{Zr}^{4+}$ to dopant ion. When an equimolar amount of trivalent ions has been added the formula of the mixed oxide is $\mathrm{A}_{2} \mathrm{~B}_{2} \mathrm{O}_{7}$ and, depending on the type of $A$ and $B$ ions, an ordered superstructure of the fluorite structure, the pyrochlore structure, is formed of which an idealized drawing is shown in Fig. 6. Pyrochlore has a unit cell, belonging to the space group $\mathrm{Fd} 3 \mathrm{~m}$, consisting of eight fluorite cells with an ordered arrangement of oxygen vacancies (one in each octant at the crystallographic position 8a). Six of the remaining oxygens are of the same crystallographic type (48f) the seventh has a different crystallographic type (8c). There is also cation ordering and the lattice is distorted in such a way that the smaller of the cations is surrounded by a distorted octahedron of oxygens, the larger by a distorted cube of oxygens.

The structure relations are shown in the phase diagram in Figs. 7 and 8 . In the $\mathrm{ZrO}_{2}-\mathrm{La}_{2} \mathrm{O}_{3}$ diagram of Fig. $7[53,54]$ the pyrochlore phase, which shows an appreciable homogeneity range, is clearly present. In the $\mathrm{ZrO}_{2}-\mathrm{Y}_{2} \mathrm{O}_{3}$ phase diagram of Fig. 8 [55, 56], by contrast, there is no indication of the existence of a phase with the pyrochlore structure. In fact, when comparing the phase diagrams of the different $\mathrm{ZrO}_{2}$ $\mathrm{Ln}_{2} \mathrm{O}_{3}$ systems [53], it is clear that the pyrochlore structure becomes less stable as the radius ratio $\mathrm{r}\left(\mathrm{Ln}^{3+}\right) / \mathrm{r}\left(\mathrm{Zr}^{4+}\right)$ decreases. As a consequence of the lanthanide contraction this means that from gadolinium onwards the pyrochlore structure is no longer formed. 
Around the corresponding composition the stable phase is then the cubic fluorite structure with a random distribution of the metal cations over the accessible sites in the structure.

Bismuth sesquioxide has a number of different structures which are all related to the fluorite structure, as shown by Harwig and Weenk [57, 58]. The $\alpha$ and $\delta$ structures are stable, but $\beta$ and $\gamma$ are metastable and are formed on cooling the $\delta$ structure. $\alpha-\mathrm{Bi}_{2} \mathrm{O}_{3}$ transforms at $1002 \mathrm{~K}$ to $\delta-\mathrm{Bi}_{2} \mathrm{O}_{3}$ which is stable up to its melting point of $1097 \mathrm{~K}$.

One of the structures proposed for $\delta-\mathrm{Bi}_{2} \mathrm{O}_{3}$, which is shown in Fig. 9, is obtained from the fluorite structure by removing, in an ordered way, two oxygen ions from opposite corners of the $\mathrm{O}_{8}$ cube in that structure.

Several other related structures have also been proposed [57, 58]. Common to them all is their derivation from the fluorite structure. As has been demonstrated by Jacobs et al. [59] computational modelling of these structures shows that the energy differences between these structures are very small, indicating that they may all be present in a dynamic equilibrium. This is also in agreement with the fact that threequarters of the total entropy change from $\alpha$ to liquid occurs during the transition $\alpha \rightarrow \delta$, which indicates a degree of disorder in the oxygen sublattice of the $\delta$ phase comparable with that in the liquid phase.

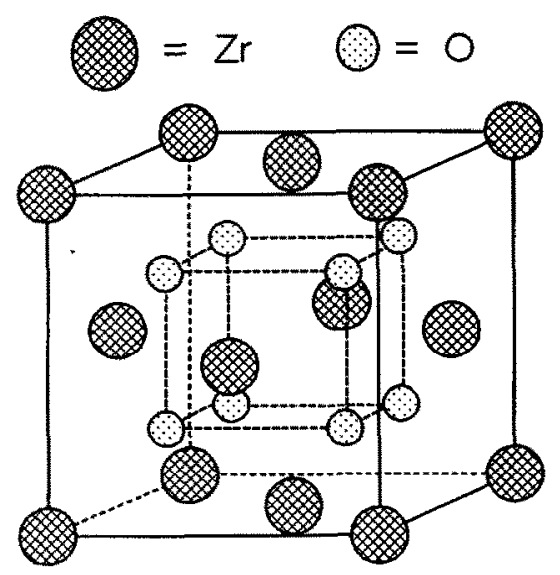

Fig. 5. Fluorite structure of $\mathrm{ZrO}_{2}$.

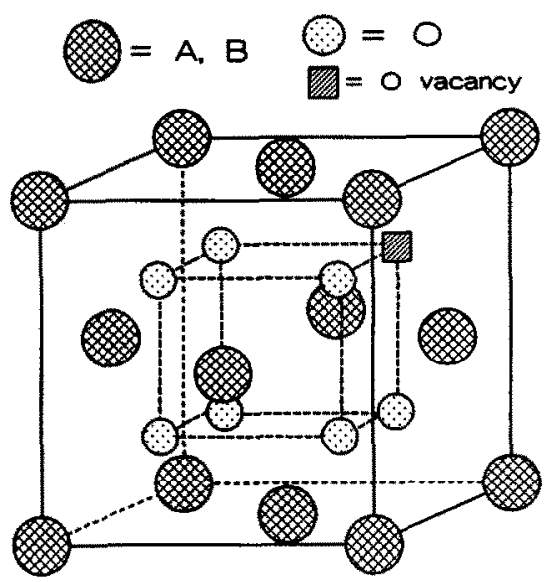

Fig. 6. Pyrochlore structure. 


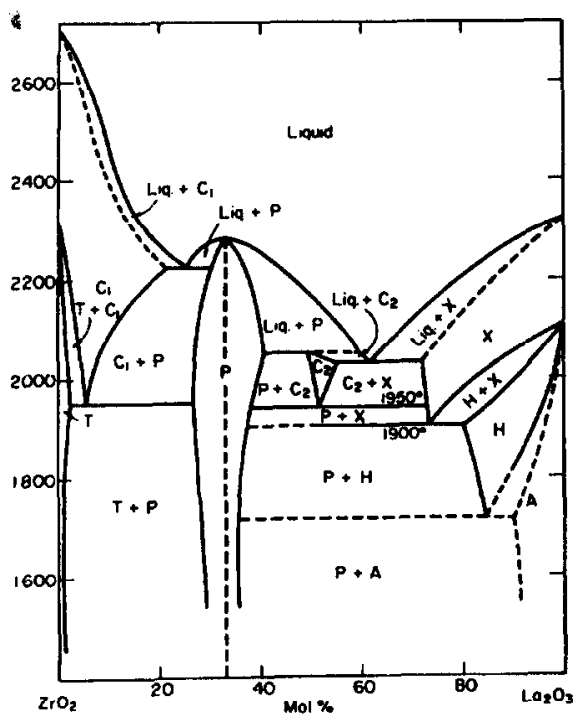

Fig. 7. Phase diagram of $\mathrm{ZrO}_{2}-\mathrm{La}_{2} \mathrm{O}_{3}$ (Reproduced with permission of the American Ceramic Society from Ref. 54).

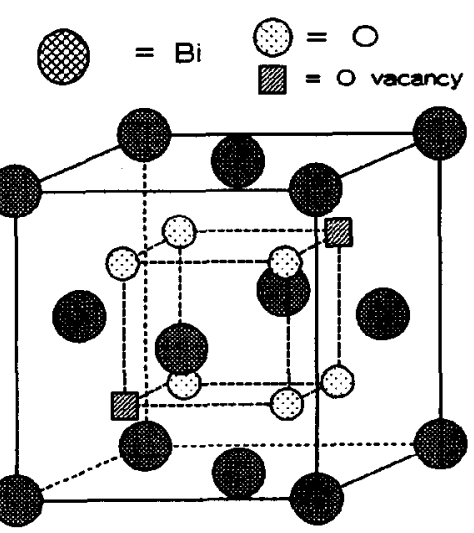

Fig. 9. Structure of $\delta-\mathrm{Bi}_{2} \mathrm{O}_{3}$. An idealized picture of $1 / 4$ of the unit cell is shown.

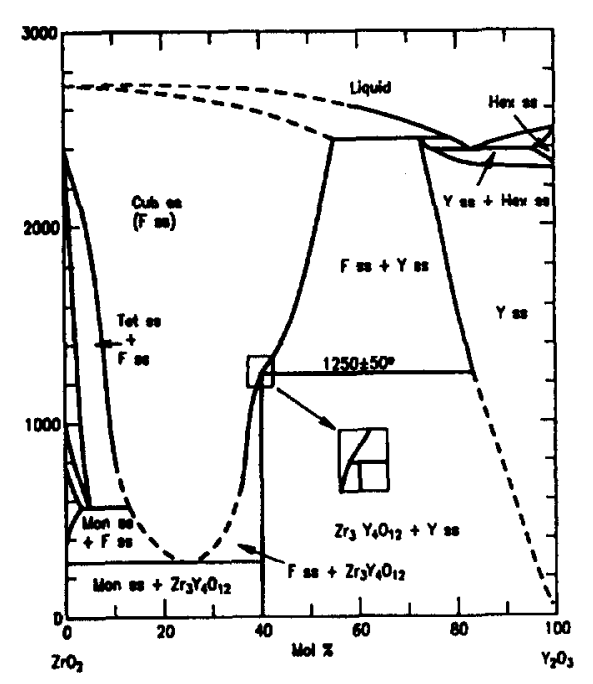

Fig. 8. Phase diagram of $\mathrm{ZrO}_{2}-\mathrm{Y}_{2} \mathrm{O}_{3}$ (Reproduced with permission of the American Ceramic Society from Ref. 56).

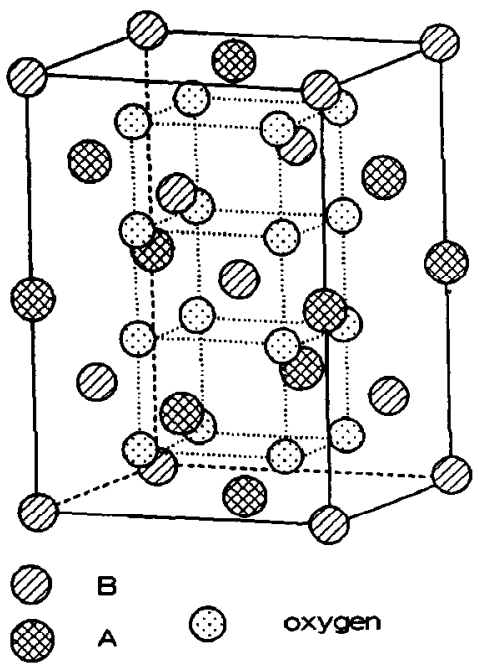

Fig. 10. Scheelite structure of composition $\mathrm{ABO}_{4}$ (idealized). 


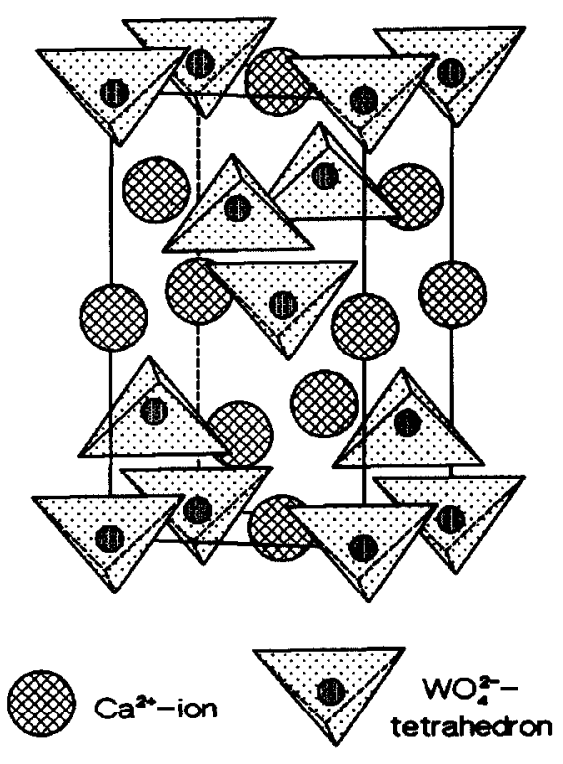

Fig. 11. Real scheelite structure.

Another important and interesting structure is that of scheelite, $\mathrm{CaWO}_{4}$, of which the idealized structure is shown in Fig. 10. This demonstrates clearly the relation to the fluorite structure. In reality the oxygen ions are shifted in such a way that the tungstate ions are tetrahedrally surrounded by oxygen ions, whereas the calcium ions are surrounded by 12 oxygen ions (see Figs. 11 and 13).

An important class of compounds within the scope of this review is formed by the bismuth molybdates. This is a series of compounds, $\mathrm{Bi}_{2} \mathrm{MoO}_{6}, \mathrm{Bi}_{2} \mathrm{Mo}_{2} \mathrm{O}_{9}$ and $\mathrm{Bi}_{2} \mathrm{Mo}_{3} \mathrm{O}_{12}$ among them. These have different structures of which that of $\mathrm{Bi}_{2} \mathrm{MoO}_{6}$ is of particular interest. This has a rather intricate structure which is schematically shown in Fig. 12.

This is a structure consisting of alternating layers of $\left[\mathrm{Bi}_{2} \mathrm{O}_{2}\right]^{2+}$, of $\mathrm{O}^{2-}$ and of $\left[\mathrm{MoO}_{2}\right]^{2+}$. In the $\left[\mathrm{Bi}_{2} \mathrm{O}_{2}\right]^{2+}$ layers there is a square pyramidal configuration of bismuth at the apex and four oxygens at the other corners. In the $\left[\mathrm{MoO}_{2}\right]^{2+}$-layers the molybdenum ions are approximately octahedrally coordinated with four oxygens in the $\mathrm{MoO}_{2}$ plane and one oxygen from the $\mathrm{O}$ plane above and one from the $O$ plane below. This structure can also be described as:

$$
\frac{\ldots . .,\left[\mathrm{Bi}_{2} \mathrm{O}_{2}\right]^{2+}, \mathrm{O}^{2-},\left[\mathrm{MoO}_{2}\right]^{2+}, \mathrm{O}^{2-},\left[\mathrm{Bi}_{2} \mathrm{O}_{2}\right]^{2+}, \mathrm{O}^{2-}, \ldots . .}{\mathrm{Bi}_{2} \mathrm{MoO}_{6}}
$$

Although the structures of $\mathrm{Bi}_{2} \mathrm{MoO}_{6}$ and of the other bismuth molybdates as usually described, looks very different from the fluorite structure, Buttrey et al. [60] have advanced arguments that the structures of the majority of the seven bismuth molybdate structures can be derived from the fluorite structure. The authors base this proposal on a detailed study of these compounds with high resolution electron microscopy and with electron and X-ray diffraction.

These structure relations are shown schematically in Fig. 13. By small displacements of the oxygen atoms as shown in Fig. 13.(b) half of the metal ions (molybdenum in the case of $\alpha-\mathrm{Bi}_{2} \mathrm{Mo}_{3} \mathrm{O}_{12}$, tungsten in $\mathrm{CaWO}_{4}$ ) become tetrahedrally 


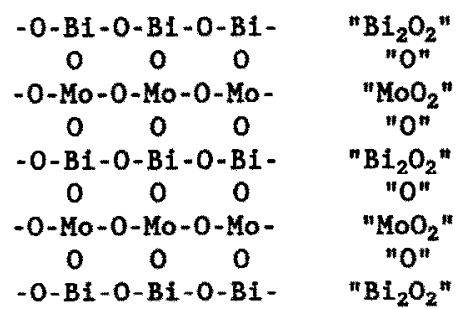

Fig. 12. Structure of $\mathrm{Bi}_{2} \mathrm{MoO}_{6}$ (schematic).

surrounded by oxygen. The compound $\alpha-\mathrm{Bi}_{2} \mathrm{Mo}_{3} \mathrm{O}_{12}$ can be written as $\mathrm{Bi}_{2 / 3} \Phi_{1 / 3} \mathrm{MoO}_{4}$ where $\Phi$ represents a vacancy: this compound is related to the scheelite structure with one third of the $A$ ions removed.

An important and extensive class of compounds is formed by the so-called perovskites which have the general formula $\mathrm{ABO}_{3}$ and of which $\mathrm{CaTiO}_{3}$ is the basis compound. In these compounds the large $A$ ion is surrounded by 12 oxygen ions, while the small B ion is octahedrally coordinated by 6 oxygens as shown in Fig. 14. In this structure substitutions are possible both on the A and on the B sites. Although most of the pure perovskite type of compounds are not ion conductors, many substituted perovskites are (see, e.g. Ref. 61).

- oxygen

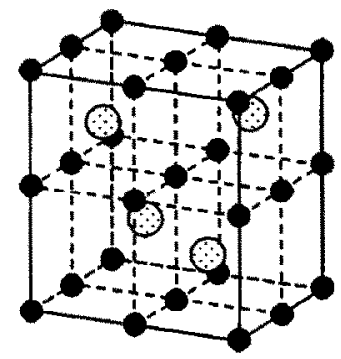

a.

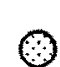

zirconium

(metal A)

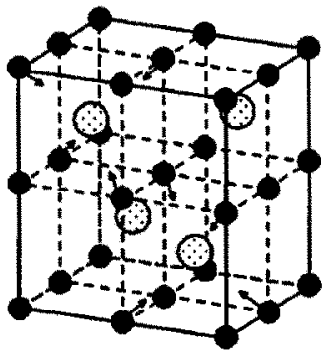

b. $\otimes$ metal B

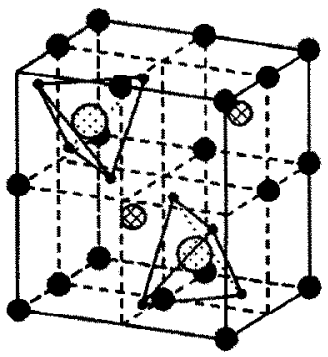

C.

Fig. 13. Relation between fluorite, scheelite and $\alpha-\mathrm{Bi}_{2} \mathrm{Mo}_{3} \mathrm{O}_{12}$ structures: a. fluorite, $b$. direction of oxygen displacements, $c$. tetrahedral coordination of half of the metal ions. 


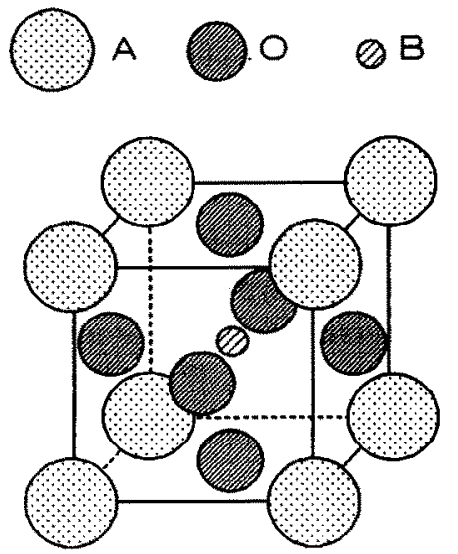

Fig. 14. Perovskite structure $\left(\mathrm{ABO}_{3}\right)$.

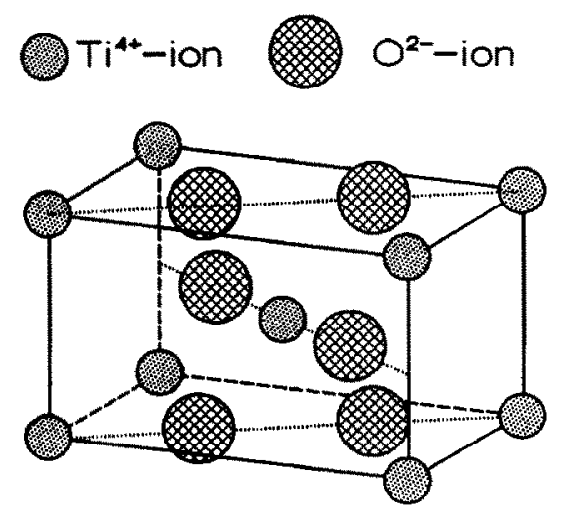

Fig. 15. Unit cell of the rutile structure with $\mathrm{c} / \mathrm{a} \approx 0.67$.

In Fig. 14 the basic structure, which is cubic, is shown. There also exist many distorted variants: tetragonal, rhombohedral, orthorhombic, monoclinic, etc. These usually arise due to relatively small shifts or rotations of the $\mathrm{BO}_{6}$ octahedra. The general properties and the structures have been reviewed by Galasso [62], by Khattak and Wang [63], by Goodenough and Longo [64] and, more recently, by Baran [65]. The defect structures and the corresponding properties have been reviewed in particular by Rao et al. [66] and by Smyth [67]. Finally the review by Tejuca et al. [68] in which the reactivity and some catalytic properties are also discussed, should be referred to, too.

To conclude the enumeration of crystal types, the rutile structure must also be mentioned. Its unit cell is shown in Fig. 15. In this structure the metal ions are surrounded by a distorted octahedron of oxygen ions, while the oxygens are coordinated to three metal ions. Although the basis compound from which these compounds are derived, $\mathrm{TiO}_{2}$, does not itself show ionic conduction several compounds with other compositions having this structure do.

\subsection{Properties}

As discussed later oxygen from the lattice is often involved in catalytic reactions. This means that a correlation may be expected between the binding energy of the oxygen ions in the lattice and their catalytic activity. As a first approximation this has led to correlations between the enthalpy of formation of oxides and their catalytic activity [69]. In these cases this enthalpy of formation is defined for the reaction: 
$\mathrm{MO}_{\mathrm{n}-1}+1 / 2 \mathrm{O}_{2} \rightarrow \mathrm{MO}_{\mathrm{n}}+\Delta \mathrm{H}_{\mathrm{f}}$

that is, for the formation from the oxide with the metal ions having the next lower valency.

For many of the oxides of the transition metals these values are available, but for oxides of metals showing only a single valency and for most of the complex oxides the only available quantity is the total heat of formation of the oxide from the metal (or metals) and oxygen. Some representative values for oxides of the types discussed here are given in Table 2 . The correlation between catalytic and thermodynamic parameters has been discussed, e.g. by Roiter et al. [70] and by Sachtler et al. [71, 72] who also found that the selectivity (in their investigation for benzaldehyde oxidation) is correlated with the differential enthalpy of reduction of the oxides studied.

Table 2

Formation enthalpies of oxides

\begin{tabular}{lll}
\hline Compound & formed from & $\Delta \mathrm{H}_{\mathrm{f}}\left(\mathrm{kJ} \cdot \mathrm{mol}(\mathrm{O})^{-1}\right)$ \\
\hline $1 / 2 \mathrm{ZrO}_{2}$ & $1 / 2 \mathrm{Zr}+1 / 2 \mathrm{O}_{2}$ & -550.0 \\
$\mathrm{~V}_{2} \mathrm{O}_{5}$ & $2 \mathrm{VO}_{2}+1 / 2 \mathrm{O}_{2}$ & -15.0 \\
$\mathrm{SnO}_{2}$ & $\mathrm{SnO}+1 / 2 \mathrm{O}_{2}$ & -296.0 \\
$2 \mathrm{CeO}_{2}$ & $\mathrm{Ce}_{2} \mathrm{O}_{3}+1 / 2 \mathrm{O}_{2}$ & -358.0 \\
$1 / 3 \mathrm{Bi}_{2} \mathrm{O}_{3}$ & $2 / 3 \mathrm{Bi}+1 / 2 \mathrm{O}_{2}$ & -191.3 \\
$1 / 4 \mathrm{PbMoO}_{4}$ & $1 / 4 \mathrm{~Pb}+1 / 4 \mathrm{Mo}+1 / 2 \mathrm{O}_{2}$ & -278.0 \\
\hline
\end{tabular}

Pankratiev [73] later proposed a correlation between the catalytic activity of oxides and the oxygen binding energy defined as the initial heat of desorption from oxide surfaces. As a good correlation is also found for reactions in which oxygen does not directly participate this author proposed that the oxygen binding energy is a measure of the cation reactivity of oxide forming elements, so that this is a rather indirect correlation.

Perhaps the lattice self potential at the oxygen site in the compounds under consideration is a better quantity with which the catalytic activity can be correlated. Some representative values for these self potentials, calculated with a program developed from that described by Van Gool and Piken [74], are given in Table 3. This calculation is based on the assumption that the oxides are purely ionic. For a single oxide the relative 
Table 3

Lattice self potential at the oxygen site(s) in some oxides

\begin{tabular}{|c|c|c|c|c|}
\hline Type & Compound & $\begin{array}{l}\text { Lattice } \\
\text { parameters ( } \AA \text {, } \\
\text { degrees) }\end{array}$ & Site & $\begin{array}{l}\text { Potential } \\
\left(\AA^{-1}\right)\end{array}$ \\
\hline Fluorite: & $\mathrm{ZrO}_{2}$ & 5.409 & & 1.505 \\
\hline \multirow[t]{2}{*}{ Perovskite: } & $\mathrm{LaCoO}_{3}$ & 3.824 & & 1.561 \\
\hline & $\mathrm{SrFeO}_{3}$ & 3.869 & & 1.669 \\
\hline \multirow[t]{4}{*}{ Scheelites*: } & $\mathrm{CaWO}_{4}$ & a,b 5.243 & & 1.502 \\
\hline & & c 11.376 & & \\
\hline & $\mathrm{BiAsO}_{4}$ & a,b 5.08 & & 1.500 \\
\hline & & c 11.7 & & \\
\hline \multirow[t]{4}{*}{ Scheelites ${ }^{+}:$} & $\mathrm{CaWO}_{4}$ & a,b 5.243 & & 1.485 \\
\hline & & c 11.376 & & \\
\hline & $\mathrm{BiAsO}_{4}$ & a,b 5.08 & & 1.537 \\
\hline & & c 11.7 & & \\
\hline \multirow[t]{3}{*}{ Pyrochlore: } & $\mathrm{Nd}_{2} \mathrm{Zr}_{2} \mathrm{O}_{7}{ }^{1}$ & 10.6836 & $48 \mathrm{f}$ & 1.579 \\
\hline & & & $8 c$ & 1.478 \\
\hline & & & $8 a^{* *}$ & 0.507 \\
\hline \multirow[t]{6}{*}{ Bismuth oxides: } & $\alpha-\mathrm{Bi}_{2} \mathrm{O}_{3}{ }^{2}$ & a 5.8496 & $4 e(1)$ & 1.447 \\
\hline & & b 8.1648 & $4 e(2)$ & 1.416 \\
\hline & & c 7.5101 & $4 e(3)$ & 1.389 \\
\hline & & B 112.977 & & \\
\hline & $\delta-\mathrm{Bi}_{2} \mathrm{O}_{3}{ }^{3}$ & 5.644 & $6 \mathrm{~d}$ & 1.344 \\
\hline & & & $2 e^{* *}$ & 1.004 \\
\hline
\end{tabular}

Notes:

* Idealized structure.

$+\quad$ Real structure.

** This site is empty in the normal structure.

1 Space group Fd3m.

2 Space group $\mathrm{P}_{1} / \mathrm{c}$.

$3 \quad$ Space group Pn3m. 
values of these self potentials are expected to be in the same order as for a real, partially covalent, oxide. In the comparison of the values for different oxides this assumption will not be valid and care is needed in comparing these self potentials for different oxides.

For the reactions and oxides considered here the lattice self potential at the oxygen site is probably a better measure than the lattice self potential of the cations used by Kung [16, 75, 76]. The latter are particularly important when the reaction is determined principally by the adsorption of the reacting molecule to the catalyst, but less so when the oxygen uptake or removal mainly determines the reaction. 


\section{PURELY ION CONDUCTING OXIDES}

The oxygen ion conductors which are best known and widely used in solid electrolyte electrochemical cells are the stabilized zirconias having the fluorite structure or, depending on the type and amount of the stabilizing cation, heat treatment and composition, the pyrochlore structure. Some typical values of the ion conductivity and its activation enthalpy of a number of oxygen ion conducting oxides are given in table 4 .

Table 4

Conductivities of some oxides with predominant ion conduction

\begin{tabular}{lccc}
\hline Compound & $\begin{array}{c}\text { temperature } \\
\left({ }^{\circ} \mathrm{C}\right)\end{array}$ & $\begin{array}{c}\text { conductivity } \\
\left(\Omega^{-1} \mathrm{~m}^{-1}\right)\end{array}$ & $\begin{array}{c}\Delta \mathrm{H}_{\text {act }} \\
\left(\mathrm{kJ}^{-1} \mathrm{~mol}^{-1}\right)\end{array}$ \\
\hline$\delta-\mathrm{Bi}_{2} \mathrm{O}_{3}$ & 1000 & 710.0 & 28 \\
$\mathrm{ZrO}_{2}+12 \% \mathrm{CaO}$ & 1000 & 5.5 & 106 \\
$\mathrm{ZrO}_{2}+9 \% \mathrm{Y}_{2} \mathrm{O}_{3}$ & 1000 & 12.0 & 77 \\
$\mathrm{ZrO}_{2}+17 \% \mathrm{Y}_{2} \mathrm{O}_{3}$ & 1000 & 68.2 & 103 \\
$\mathrm{Nd}_{2} \mathrm{Zr}_{2} \mathrm{O}_{7}$ & 1000 & 0.03 & 76 \\
$\mathrm{Sm}_{2} \mathrm{Zr}_{2} \mathrm{O}_{7}$ & 1000 & 0.42 & 67 \\
$\mathrm{Gd}_{2} \mathrm{Zr}_{2} \mathrm{O}_{7}$ & 1000 & 1.99 & 112 \\
$\mathrm{Ce}_{0.77} \mathrm{Gd}_{0.23} \mathrm{O}_{1.885}$ & 997 & 0.33 & \\
\hline
\end{tabular}

In many cases the ion conductivities of these materials are relatively small at low temperatures, e.g. 400 to $600{ }^{\circ} \mathrm{C}$. This means that they will not be very active in catalytic reactions in which lattice oxygen plays a role. This is in particular a disadvantage for reactions in which the selectivity is important; the selectivity often decreases strongly at high temperatures, due to the occurrence of gas phase reactions, among other reasons.

Increases in ion conductivity can be obtained by introducing [20]:

- $\quad$ different oxygen sublattices with different binding energies;

- $\quad$ rapid diffusion paths;

- $\quad$ high ionic polarizabilities.

The first two are found, for instance in oxides with the pyrochlore structure [77, 78, 79]. The latter two are found in bismuth containing materials, see e.g. Refs. 80, 81. Of the bismuth oxides the high temperature form, $\delta-\mathrm{Bi}_{2} \mathrm{O}_{3}$, is in fact the oxygen ion conductor with the highest known oxygen ion conductivity, but this form is unfortunately only stable between 1002 and $1097 \mathrm{~K}$. This means that for practical applications doping with stabilizing agents is necessary. Up to now all these stabilizing agents have unfortun- 
ately led to a decrease in ion conductivity.

During heat treatment of gadolinium-zirconium oxides $\left(\mathrm{Gd}_{\mathrm{x}} \mathrm{Zr}_{1-\mathrm{x}} \mathrm{O}_{2-\mathrm{x} / 2}\right)$ microdomains of the ordered pyrochlore structure are formed; first in a matrix of unordered fluorite, but later these domains come into contact, forming anti-phase boundaries in which continuous, fast diffusion pathways are present [82].

In mixed systems, with $\mathrm{Bi}_{2} \mathrm{O}_{3}$ as one component and one of $\mathrm{Ta}_{2} \mathrm{O}_{5}, \mathrm{Nb}_{2} \mathrm{O}_{5}, \mathrm{~V}_{2} \mathrm{O}_{5}$, $\mathrm{MoO}_{3}$, and $\mathrm{WO}_{3}$ as the other, wide compositional ranges of solid solutions exist with an extensive variety of ordered phases showing well-defined superstructures [83]. Several of these systems show interesting catalytic and other solid state properties. The structures of many of these compounds are related to the fluorite structure as has already been mentioned earlier for many of the bismuth molybdates [60] (see Fig. 13 in Section 4.1). It is suggested that the high mobility of lattice oxygen in these compounds, as well as the ease of insertion of oxygen into, and its release from, the solid catalyst is connected with the cation and/or anion defects which are easily accommodated in the fluorite framework.

Kudo and Obayashi [84] studied the oxygen ion conducting properties of the lanthanide substituted cerium oxides $\mathrm{Ce}_{1-\mathrm{x}} \mathrm{Ln}_{\mathrm{x}} \mathrm{O}_{2-\mathrm{x} / 2}$ with $\mathrm{Ln}=\mathrm{Y}, \mathrm{La}, \mathrm{Nd}, \mathrm{Sm}, \mathrm{Eu}, \mathrm{Gd}$, $\mathrm{Dy}, \mathrm{Ho}, \mathrm{Er}, \mathrm{Yb}$. Above $873 \mathrm{~K}$ oxygen ions are the majority charge carriers in these compounds with ionic transference numbers $t_{\text {ion }}>0.95$. Below $873 \mathrm{~K}$ they show different amounts of mixed conductivity, which was not studied in detail. The Arrhenius plots show two straight lines intersecting at a temperature in the neighbourhood of $923 \mathrm{~K}$. The activation enthalpies in the low temperature region are of the order of 90.7 to 110 $\mathrm{kJ} \cdot \mathrm{mol}^{-1}$; in the high temperature region they are 75.3 to $89.7 \mathrm{~kJ} \cdot \mathrm{mol}^{-1}$. No explanation could be given for the two different activation enthalpies, but the present authors suppose that this might be due to an increasing electronic contribution to the conductivity at lower temperatures. All compounds show the fluorite structure up to about $\mathbf{x}=0.5$.

Cook and Sammell $[85,86]$ recently proposed an interesting method for selecting perovskite compositions with a high ion and a low electronic conductivity. Because the band gap, which must be as large as possible to avoid a contribution from electronic conductivity, is correlated with the enthalpy of formation, this should be relatively large. Furthermore the average metal-oxygen bond energy should be not too large, the degree of openness, or free volume, of the lattice should be large and the critical radius, $r_{c}$, of the saddle point formed by two $A$ ions and one $B$ ion should be relatively large. On this basis the compounds $\mathrm{BaTb}_{0.9} \mathrm{In}_{0.1} \mathrm{O}_{3}, \mathrm{CaCe}_{0.9} \mathrm{Gd}_{0.1} \mathrm{O}_{3}$ and $\mathrm{CaCe}_{0.9} \mathrm{Er}_{0.1} \mathrm{O}_{3}$ were selected and prepared. These compounds were indeed found to be good oxygen ion conductors with relatively low ( 35 to $53 \mathrm{~kJ} \cdot \mathrm{mol}^{-1}$ ) activation enthalpies for the conductivity. 


\section{MIXED CONDUCTING OXIDES}

A number of mixed conducting oxide systems are given in table 5, together with typical values of their conductivities, ionic transference numbers and activation enthalpies of the two contributions to the conductivity.

Table 5

Properties of mixed conducting oxides

\begin{tabular}{|c|c|c|c|c|c|}
\hline Compound & $\begin{array}{l}\text { temp } \\
\left({ }^{\circ} \mathrm{C}\right)\end{array}$ & $t_{\text {ion }}$ & $\begin{array}{l}\text { total } \\
\text { conductivity } \\
\left(\Omega^{-1} \mathrm{~m}^{-1}\right) \\
\end{array}$ & $\begin{array}{l}\Delta \mathbf{H}_{\mathrm{acct}}(\mathrm{ion}) \\
\left(\mathbf{k J} \cdot \mathrm{mol}^{-1}\right)\end{array}$ & $\begin{array}{l}\Delta \mathrm{H}_{\mathrm{act}}(\mathrm{el}) \\
\left(\mathrm{kJ} \cdot \mathrm{mol}^{-1}\right)\end{array}$ \\
\hline $\mathrm{Ce}_{0.7} \mathrm{~Tb}_{0.3} \mathrm{O}_{2-\mathrm{x}}$ & 600 & 0.18 & 1.47 & 115 & 52 \\
\hline$\left(\mathrm{Bi}_{0.7} \mathrm{~Tb}_{0.3}\right)_{2} \mathrm{O}_{3+\mathrm{y}}$ & 600 & 0.67 & 1.5 & 120 & 94 \\
\hline$\left(\mathrm{Tb}_{0.4} \mathrm{Gd}_{0.6}\right)_{2} \mathrm{Zr}_{2} \mathrm{O}_{7}$ & 800 & 0.6 & 0.31 & 136 & \\
\hline $\mathrm{La}_{0.2} \mathrm{Sr}_{0.8} \mathrm{Co}_{0.8} \mathrm{Fe}_{0.2} \mathrm{O}_{3}$ & 800 & 0.007 & 180.0 & & \\
\hline $\mathrm{CaTi}_{0.8} \mathrm{Fe}_{0.2} \mathrm{O}_{3}$ & 800 & 0.28 & 0.1 & & \\
\hline $\mathrm{Bi}_{2} \mathrm{Mo}_{2} \mathrm{O}_{9}$ & 600 & 0.51 & 0.324 & 180 & 116 \\
\hline
\end{tabular}

One of the major reasons for the interest in mixed conductors is the possibility of using these as electrodes in solid oxide electrochemical cells. If metal electrodes or oxide electrodes with purely electronic conduction are applied, the charge transfer reaction only takes place at the three phase line: electrode / electrolyte / gas. With a mixed conducting electrode the charge transfer reaction occurs over the whole surface because oxygen ions and electrons are mobile in such a material, so that polarization losses are reduced. This is shown schematically in Fig. 16.

$\delta-\mathrm{Bi}_{2} \mathrm{O}_{3}$ has already been mentioned as the ion conductor with the highest known oxygen ion conductivity. $\alpha-\mathrm{Bi}_{2} \mathrm{O}_{3}$ on the other hand, as shown by Harwig and Gerards [87], is a nearly pure electronic conductor at low temperatures, with electron holes being the mobile species. Upon increasing the temperature an increasing contribution of ion conduction becomes observable above $923 \mathrm{~K}$ and close to the $\alpha \rightarrow \delta$ transition temperature $(1002 \mathrm{~K})$ the ionic transference number is of the same order of magnitude as the electronic transference number. Upon cooling from above $1002 \mathrm{~K}$ the $\beta$ or $\gamma$ forms of $\mathrm{Bi}_{2} \mathrm{O}_{3}$ are formed as intermediate phases before $\alpha . \beta-\mathrm{Bi}_{2} \mathrm{O}_{3}$ and $\gamma-\mathrm{Bi}_{2} \mathrm{O}_{3}$ are both ion conductors, but their conductivities are near to three orders of magnitude smaller than that of $\delta-\mathrm{Bi}_{2} \mathrm{O}_{3}$.

Perovskite type oxides can show different kinds of conduction. $\mathrm{LaCrO}_{3}$ and $\mathrm{LaFeO}_{3}$ for example are insulators, while $\mathrm{SrCrO}_{3}$ and $\mathrm{SrFeO}_{3}$ are metallic conductors. 
Doped compounds, like the nickelites $\mathrm{La}_{1-\mathrm{x}} \mathrm{M}_{\mathrm{x}} \mathrm{NiO}_{3}$ and cobaltites, $\mathrm{La}_{1-\mathrm{x}} \mathrm{M}_{\mathrm{x}} \mathrm{CoO}_{3}$ show mixed conduction and have high electronic and ion conductivities and are presently being investigated as electrode materials [18]. Torrance et al. [88] have proposed a simple ionic model which is able to explain the reasons for metallic or insulating behaviour. It is concluded from this model that these large differences in conductivity behaviour are mainly due to differences in the ionization energies of the transition metal ions present in these compounds. In this way, for example, it can be explained that the compounds $\mathrm{SrCrO}_{3}$ and $\mathrm{SrFeO}_{3}$, with 4-valent transition metal ions, show metallic conduction whereas $\mathrm{LaCrO}_{3}$ and $\mathrm{LaFeO}_{3}$, with 3-valent transition metal ions, are insulating (or better: semiconducting).
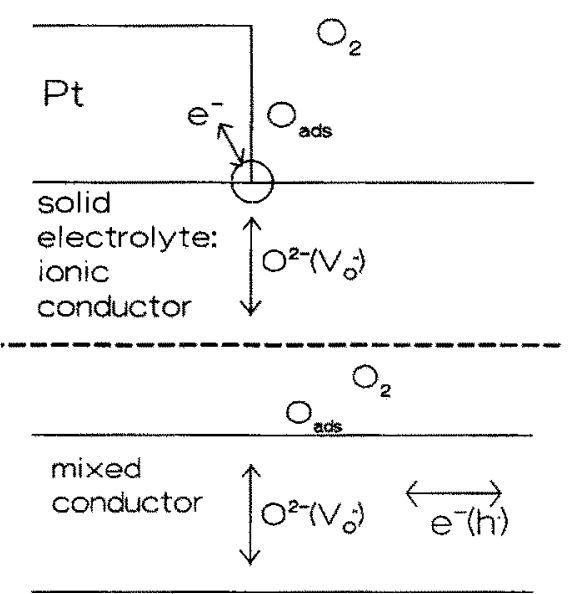

solid
electrolyte:
ionic
conductor $\mathrm{O}^{2-\left(V_{0}\right)}$

Fig. 16. Comparison of processes with metal electrode and mixed conducting oxide electrode.

Perovskites of the type $\mathrm{SrMnO}_{3-\delta}$ are good electronic conductors but, as shown by Iwahara et al. [89] these are also ion conductors. Using a measuring method in which the electronic contribution to the current was blocked, these authors were able to determine the ion conductivity, which was observed to be two to three orders of magnitude smaller than the electronic conductivity. On the other hand the ion conductivity itself is of the same order of magnitude as that in stabilized zirconia. Similar observations were made on the related materials $\mathrm{SrMn}_{1-\mathrm{x}} \mathrm{Co}_{\mathrm{x}} \mathrm{O}_{3-\delta}$ and $\mathrm{Sr}_{1-\mathrm{x}} \mathrm{Na}_{\mathrm{x}} \mathrm{MnO}_{3-\delta}$.

Oxygen ion conduction depends on the presence of interstitial oxygen ions or of oxygen vacancies. The latter are in general only found in doped perovskites such as $\mathrm{Ln}_{1-\mathrm{x}} \mathrm{M}_{\mathrm{x}} \mathrm{TO}_{3-\delta}$, where $\mathrm{Ln}$ is a lanthanide ion, $\mathrm{M}$ a bivalent metal ion, e.g. an alkaline earth metal substitutionally present on $\mathrm{Ln}$ sites and $\mathrm{T} a$, nominally, trivalent transition metal ion. In this case the electroneutrality condition is:

When the fourth ionization energy of the transition metal ion is not too high, this kind of doping may also lead to charge compensation by the formation of electron holes, giving the electroneutrality condition:

This causes a decrease in the ion conductivity but an increase in (p-type) 


$$
\left[\mathrm{h}^{*}\right]+2\left[\mathrm{~V}_{\mathrm{O}}^{*}\right]=\left[\mathrm{M}_{\mathrm{Ln}}{ }^{\prime}\right]
$$

electronic conductivity. In this case the oxygen vacancy and hole concentrations, and thus also the corresponding conductivities, depend on the oxygen partial pressure: increasing $\mathrm{p}_{\mathrm{O} 2}$ leading to decreased ionic and increased electronic contributions to the conductivity.

Mizusaki et al. [90] investigated the nonstoichiometry in perovskite-type oxides of the type: $\mathrm{La}_{1-x} \mathrm{Sr}_{\mathrm{x}} \mathrm{CoO}_{3-\delta}$. They observed the formation both of $\mathrm{Co}^{4+}$-ions and of oxygen vacancies. The nonstoichiometry parameter $\delta$ became measurable above 450 to $550 \mathrm{~K}$ and increased with temperature. For $\mathrm{x}<0.2$ the formation of $\mathrm{Co}^{4+}$ gave the main contribution at high oxygen partial pressure, while $\delta$ deviated significantly from 0 at low $\mathrm{p}_{\mathrm{O} 2}$. For $0.2<\mathrm{x}<0.7$ the formation of oxygen vacancies gave the main contribution to nonstoichiometry at all temperatures. Above $x=0.7$ there was again an increasing contribution of the formation of $\mathrm{Co}^{4+}$. Both the dependence of $\delta$ on $\mathrm{p}_{\mathrm{O}_{2}}$ and the small electronic contribution to the entropy point to metallic or semimetallic electronic conduction. The dependence of the entropy on $\delta$ is determined by the configurational entropy of the oxygen vacancies and this means that the vacancies are randomly distributed over the oxygen sublattice sites.

These and similar materials are especially investigated as cathode materials, e.g. in solid oxide fuel cells. This means that the catalytic activity for the reaction:

$$
1 / 2 \mathrm{O}_{2}+\mathrm{V}_{\mathrm{O}}^{\infty} \rightarrow \mathrm{O}_{\mathrm{O}}+2 \mathrm{~h}^{*}
$$

is of particular interest.

The chemical diffusion coefficient of oxygen ions in a $\mathbf{L a}-\mathrm{Sr}$ manganite has been determined to be of the order of $10^{-7} \mathrm{~cm}^{2} \cdot \mathrm{s}^{-1}$ at temperatures around $800^{\circ} \mathrm{C}$ and oxygen partial pressures between 0.2 and $10^{-8} \mathrm{~atm}$ by Belzner et al. [91], using the potentiostatic step method.

The tracer diffusion coefficients of oxide ions $\mathrm{D}_{\mathrm{O}}^{*}$ in the perovskites $\mathrm{La}_{1-x} \mathrm{Sr}_{\mathrm{x}} \mathrm{CoO}_{3-\delta}(\mathrm{x}=0.1)$ and $\mathrm{La}_{1-\mathrm{x}} \mathrm{Sr}_{\mathrm{x}} \mathrm{FeO}_{3-\delta}(\mathrm{x}=0.1,0.25$ and 0.4$)$ were determined by Ishigaki et al. [92] and from this the diffusion coefficient, $D_{V}$, of the oxygen vacancies was estimated. Some typical values are given in Table 6 and these are of the same order of magnitude as those found in fluorite type oxides, e.g., $\approx 8 \times 10^{-4} \mathrm{~cm}^{2} . \mathrm{s}^{-1}$ for $\mathrm{Ca}$ and $\mathrm{Mg}$ stabilized $\mathrm{ZrO}_{2}[93]$ and $\approx 5 \times 10^{-6} \mathrm{~cm}^{2} . \mathrm{s}^{-1}$ for $\mathrm{Ca}$ doped $\mathrm{CeO}_{2}$ [94].

Teraoka et al. [61] reported the mixed ionic-electronic conductivity of $\mathrm{La}_{1-\mathrm{x}} \mathrm{Sr}_{\mathrm{x}} \mathrm{Co}_{1-\mathrm{y}} \mathrm{Fe}_{\mathrm{y}} \mathrm{O}_{3-\delta}$ oxides, which are also perovskite-type compounds. The electronic and ionic conductivities, $\sigma_{\text {ion }}$ and $\sigma_{\mathrm{el}}$, were measured separately using four-probe ion dc and ordinary four-probe techniques. The electronic conductivities of these compounds were observed to depend only slightly on temperature, $\mathrm{p}_{\mathrm{O} 2}$ or substitution and were 1 to $2 \times 10^{4} \Omega^{-1} \cdot \mathrm{m}^{-1}$. 
The ion conductivities were found to be a factor $10^{2}$ to $10^{4}$ smaller, leading to an ionic transference number $t_{\text {ion }}$ of the order of $10^{-2}$ to $10^{-4}$. The highest ion conductivities were observed in $\mathrm{He}$ (i.e. at low $\mathrm{P}_{\mathrm{O} 2}$ ), for large $\mathrm{x}$ and for small $\mathrm{y}$. The activation enthalpy, assuming an oxygen ion vacancy mechanism for the oxide ion conductivity, was found to be $80 \pm 20 \mathrm{~kJ} \mathrm{~mol}^{-1}$, which is reasonably close to the values for the activation enthalpy for migration of oxygen vacancies in $\mathrm{LaCoO}_{3}$ and $\mathrm{LaFeO}_{3}$ found by Ishikagi [95, 96].

Table 6

Diffusion coefficients of oxygen vacancies in perovskites

\begin{tabular}{ccc}
\hline $\mathrm{T}\left({ }^{\circ} \mathrm{C}\right)$ & $\mathrm{x}$ & $\mathrm{D}_{\mathrm{V}}\left(\mathrm{cm}^{2} . \mathrm{s}^{-1}\right)$ \\
\hline $\mathrm{La}_{1-\mathrm{x}} \mathrm{Sr}_{\mathrm{x}} \mathrm{CoO}_{3-\delta}$ & & \\
1000 & 0.1 & $9.76 \times 10^{-6}$ \\
950 & & $7.56 \times 10^{-6}$ \\
900 & & $5.55 \times 10^{-6}$ \\
$\mathrm{La}_{1-\mathrm{x}} \mathrm{Sr}_{\mathrm{x}} \mathrm{FeO}_{3-\delta}$ & & \\
1050 & 0.1 & $1.21 \times 10^{-5}$ \\
1000 & & $7.41 \times 10^{-6}$ \\
950 & & $4.59 \times 10^{-6}$ \\
& & \\
1050 & 0.25 & $2.36 \times 10^{-5}$ \\
1000 & & $1.32 \times 10^{-5}$ \\
950 & & $6.18 \times 10^{-6}$ \\
& & \\
1000 & 0.4 & $1.95 \times 10^{-5}$ \\
\hline
\end{tabular}

Priestnall and Steele [97] investigated $\mathrm{La}_{1.7} \mathrm{Sr}_{0.3} \mathrm{CuO}_{3.85+\delta}$ as a possible electrode material for solid oxide fuel cells and were able to show that the material is highly compatible with an yttria stabilized zirconia electrolyte and is promising as a cathode for this application.

Compounds of the type $\left(\mathrm{In}_{2} \mathrm{O}_{3}\right)_{x}\left(\mathrm{PrO}_{1.83}\right)_{y}\left(\mathrm{ZrO}_{2}\right)_{z}$ were also investigated for the same purpose [98]. The bcc solid solution with $x=0.5, y=0.2, z=0.3$ shows the highest electronic transference number of 0.9999 , whereas the two phase system $(\mathrm{bcc}+\mathrm{fcc}$ ) with $x=0.3, y=0.1, z=0.6$ has the highest ion transference number ranging from 0.09 at $900 \mathrm{~K}$ to about 0.7 at $1300 \mathrm{~K}$. These compounds showed good behaviour when used as a 
cathode material.

In an investigation of $\mathrm{La}_{1-x} \mathrm{Sr}_{x} \mathrm{MnO}_{3}$ as cathode material for fuel cells, Hammouche et al. $[99,100]$ observed that these compounds have a perovskite structure with an expansion coefficient not far from that of stabilized zirconia so that the compatibility of these materials is good. Furthermore, it was observed that the conduction mechanism changes from that of a semiconductor to metallic for $x=0.5$. Electrochemical measurements showed that the material has a high activity both in oxygen evolution and reduction. Moreover, this oxygen reaction catalytic activity increases with $x$. In a later investigation [101] these authors observed that at low cathodic polarization the material behaves as an electronic conductor, similar to a metal electrode. However, at high polarization a sudden acceleration of the electrode kinetics is observed. The authors attributed this to the formation of oxygen vacancies in the material as a consequence of an electrocatalytic effect, which is caused by the cathodic reduction of the manganite by which oxygen vacancies are formed. This leads to mixed conduction of the manganite so that the reaction is no longer limited to the triple-contact line but occurs over a much larger part of the electrode surface. Similar effects are discussed in Section 12.10. This is in agreement with the scheme shown in Fig. 16. Rapid oxygen diffusion through the material is the main characteristic of this newly observed behaviour. From impedance measurements the authors deduced an oxygen vacancy diffusion coefficient in the manganite (at a value of $x$ which was not given) of $4 \times 10^{-8} \mathrm{~cm}^{2} \cdot \mathrm{s}^{-1}$ at $960^{\circ} \mathrm{C}$.

Upon doping, with two- or threevalent cations, the ion conductor $\mathrm{CeO}_{2}$ is converted into a mixed conductor [102, 103]. Due to the high electronic and ion conductivities of some of these materials they are well suited, like the above-mentioned perovskites, as mixed conducting electrodes for oxygen exchange and probably also for other oxidation/reduction reactions in electrochemical systems.

In many cases mixed conductivity arises in ion conducting oxides by doping with a mixed valence ion. Boukamp et al. [104] studied $\mathrm{CeO}_{2}$ and $\mathrm{Bi}_{2} \mathrm{O}_{3}$ doped with terbium oxide. The ionic transference numbers vary from values of 0.1 to 0.4 at $500{ }^{\circ} \mathrm{C}$ to 0.8 to 1.0 at $900^{\circ} \mathrm{C}$, this increase being connected with the higher activation enthalpy for the ion conductivity. The total conductivities are rather high namely 0.1 to $1 \Omega^{-1} \mathrm{~m}^{-1}$. It was thus suggested that these oxides are promising candidates for mixed-conducting oxygen electrode layers.

Doping of yttria stabilized zirconia with titania also gives mixed conducting materials [?, 105], which were studied in connection with their possible use as cathode materials in solid oxide fuel cells too. It is suggested that in these materials the titanium induces electronic conduction along the grain boundaries.

The electrical properties of a number of bismuth molybdates: $\mathrm{Bi}_{6} \mathrm{Mo}_{2} \mathrm{O}_{15}$, $\alpha-\mathrm{Bi}_{2} \mathrm{Mo}_{3} \mathrm{O}_{12}, \beta-\mathrm{Bi}_{2} \mathrm{Mo}_{2} \mathrm{O}_{9}$ and $\gamma-\mathrm{Bi}_{2} \mathrm{MoO}_{6}$ were studied by Boon and Metselaar [106, $107,108]$. With the exception of $\alpha-\mathrm{Bi}_{2} \mathrm{Mo}_{3} \mathrm{O}_{12}$, which is an electronic conductor 


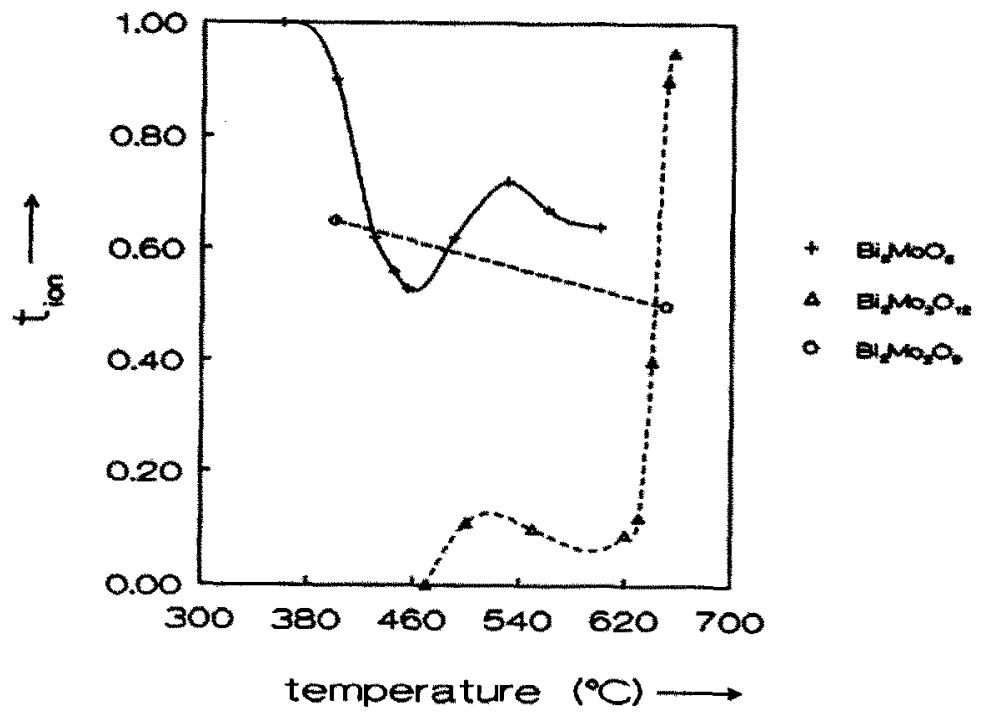

Fig. 17. Ionic transference numbers of bismuth molybdates as a function of temperature.

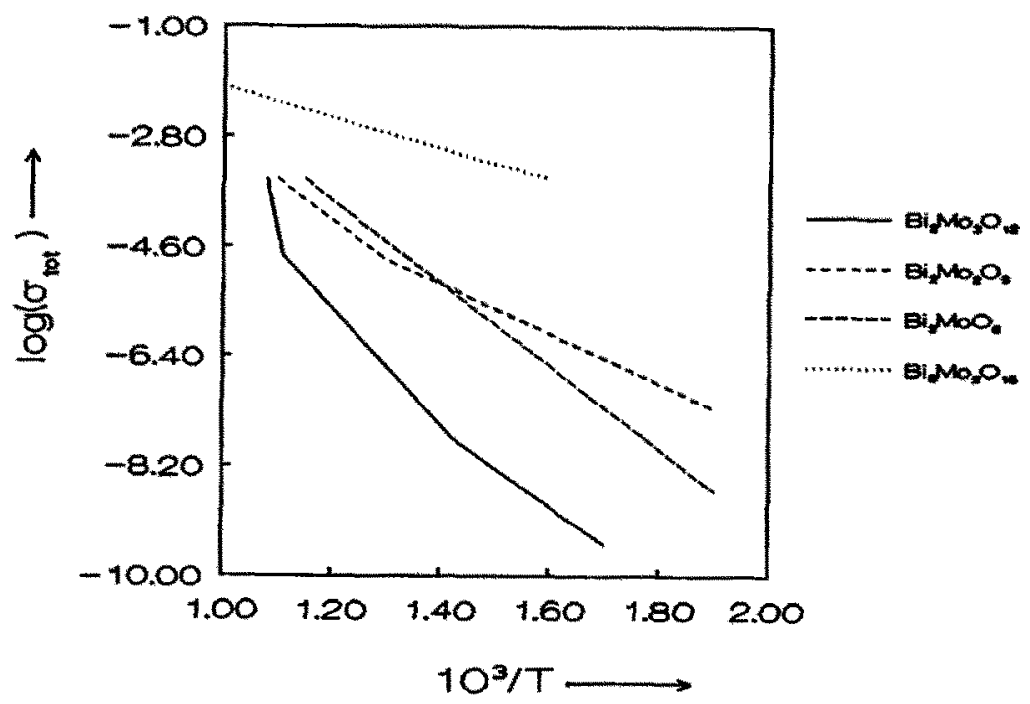

Fig. 18. Total conductivities of bismuth molybdates as a function of temperature. 
nearly up to its melting point, the others are mixed conductors. The ionic transference numbers are a function of the temperature as shown in Fig. 17. The total conductivities are reasonably high, even at temperatures as low as $300^{\circ} \mathrm{C}$ as shown in Fig. 18.

Golunski and Nevell [109] investigated the conduction properties of uranium-antimony oxide catalysts which are active and selective oxidation catalysts as shown, for example by Grasselli and Suresh [110], see also Ref. 111. In the catalyst, which consists mainly of $\mathrm{USb}_{3} \mathrm{O}_{10}$, with small proportions of $\mathrm{Sb}_{2} \mathrm{O}_{4}$ and $\mathrm{USbO}_{5}$, mixed conduction occurs. In $\mathrm{USbO}_{5}$ electronic conduction occurs only after reduction with hydrogen or propene. Ion conduction is induced after treatment in oxygen. It is suggested that in the catalytic reactions both charged oxygen vacancies and interstitial oxygen ions are involved and that the latter give rise to selective oxidation of alkenes.

A special application of mixed conductors in catalysis has been discussed by Ovenston et al. [112, 113]. These authors studied the conduction properties of tungsten bronzes and titanates in connection with the possibility of using these materials for RF heating of catalysts for the conversion of heavy oils and residues. The materials were observed to act as efficient catalysts for the steam reforming of heavy oils at temperatures of around $1000 \mathrm{~K}$. 


\section{SUREACE PROPERTIES OF OXIDES}

\subsection{Pure oxides, surface structure}

Due to the asymmetry of the surroundings of the ions in the surface layer of a crystalline solid, as a consequence of the broken periodicity perpendicular to the surface, ions may depart from the ideal lattice positions. This minimizes their (Gibbs) free energy. The calculation of these displacements and of the accompanying energy effects has been reviewed recently by Stoneham and Tasker [114].

Table 7

Surface energies of $\mathrm{UO}_{2}$ in $\mathrm{J}^{-\mathrm{m}^{-2}}$

\begin{tabular}{lll}
\hline Plane & $\begin{array}{l}\text { Surface energy } \\
\text { before relaxation }\end{array}$ & $\begin{array}{l}\text { Surface energy } \\
\text { after relaxation }\end{array}$ \\
\hline$(111)$ & 1479 & 1069 \\
$(110)$ & 3148 & 1539 \\
\hline
\end{tabular}

As an example some results from Tasker [115] of calculations on $\mathrm{UO}_{2}$, which has the fluorite structure, are summarized in Tables 7 and 8 . The energy changes due to the ionic displacements are significant, though the shifts themselves are rather small compared with the interionic distances, being at most a few percent of these.

Table 8

Ionic relaxations at (111) and (110) surfaces of $\mathrm{UO}_{2}$ in units of the lattice constant $(0.2734 \mathrm{~nm})$

\begin{tabular}{|c|c|c|c|c|c|}
\hline Plane & Direction & $\mathrm{C}^{+}$ & $\mathbf{S}^{+}$ & $\mathrm{C}^{-}$ & $\mathrm{s}^{-}$ \\
\hline (111) & perpendicular & -0.011 & 0.0149 & 0.041 & 0.0096 \\
\hline$(110)$ & perpendicular & 0.0267 & 0.0489 & 0.0317 & 0.0121 \\
\hline (110) & lateral & & & 0.0267 & 0.0489 \\
\hline
\end{tabular}

In the calculations the so-called shell and core model is used for the ions to allow for polarization in the inhomogeneous electric field near to the surface. In this model the ions are treated as consisting of a shell with a charge opposite to the core. The core and 
the shell are coupled by a spring interaction of the form:

$$
V(x)=1 / 2\left(k_{1} x^{2}+k_{2} x^{4}\right)
$$

where $x$ is the displacement of the shell relative to the core and $k_{1}$ and $k_{2}$ are dependent on the type of the ion. In general only the harmonic term is required.

For further details on the model parameters and the methods of the calculations reference must be made to the literature $[114,115]$.

\subsection{Segregation and surface composition}

An important factor in the surface properties of oxides is the possibility of segregation towards the surface leading to a difference in bulk and surface composition and properties. The reason for this is the lowering of the surface free energy as a consequence of the changed composition. Several recent reviews [116, 117, 118] treat the segregation phenomena in oxidic materials. The energetics of segregation has been discussed by Wynblatt and McCune [119] and the application of computational modelling to segregation by Egdell and Mackrodt [120]. Segregation may exert an important effect on catalysis due to the formation of a surface layer, possibly with different catalytic properties than the original, unchanged surface.

Segregation can also be due to the interaction of the gas phase with the solid oxide, causing changes in both the composition and structure of the near surface layer. One important influence is the space charge which may develop in a solid when adsorption takes place. This is discussed in somewhat more detail in the next section.

The interaction between oxide and gas can also lead to the formation of electric fields due to defect segregation, which may influence the diffusive transport and through this the gas-solid heterogeneous kinetics [121, 122]. In yttria stabilized zirconia the occurrence of yttrium segregation was detected by Winnubst et al. [123, 124, 125]. The interaction between gaseous oxygen and the surface of yttria stabilized zirconia was studied by Nowotny et al. [126], at $780{ }^{\circ} \mathrm{C}$ and $\mathrm{p}_{\mathrm{O} 2}$ between $1.74 \times 10^{2}$ and $4.3 \times 10^{4} \mathrm{~Pa}$, using in situ surface potential measurements. After quenching from 1300 to $780^{\circ} \mathrm{C}$ the oxygen exponent was found to be $1 / 4$, corresponding with the formation of doubly ionized oxygen vacancies (see Eqn. (12)). Annealing for a long time at $780^{\circ} \mathrm{C}$ led to a change in the oxygen exponent to $1 / 2$, indicating a change in the defect structure and/or the composition of the surface region. Using SIMS it was shown that impurities, in particular $\mathrm{Al}$ and $\mathrm{Ca}$, which were present in the bulk at a concentration below the detection limit, were highly enriched (concentrations of several at. $\%$ ) at the surface. Even though no detailed explanation is given, the change in exponent of the partial pressure of oxygen is ascribed to this segregation. It is clear that a large influence on the catalytic properties of an oxide in which it occurs can be expected and may well mask any influence of the bulk properties.

In $\mathrm{Sn}-\mathrm{Sb}-\mathrm{O}$ mixed catalysts calcination leads to segregation of antimony to the 
external surface [127], which is reflected in the catalytic properties. Catalysts treated at low temperatures show, just as $\mathrm{SnO}_{2}$ does, a decrease in selectivity in propene oxidation to acrolein with increasing temperature. Materials treated at high temperatures, on the other hand, show the opposite trend, just as is observed for $\mathrm{Sb}_{2} \mathrm{O}_{4}$. It was proposed that an epitaxial layer of antimony oxide is formed on these catalysts. The sensitivity to the surface structure of the catalysts follows from the differences in the selectivity patterns of the allylic oxidations of butenes, 0 -xylene and propene, the last one being especially demanding with respect to a proper surface structure.

One possible way to influence the surface composition of materials is by ion implantation [128, 129, 130] which may alter both the conducting and the catalytic properties of a surface. In most of the cases mentioned by these authors noble metals are implanted, which may give rise to noble metal catalysis rather than to catalysis by a mixed or ion conducting oxide.

\subsection{Surface charges and fields}

When chemisorption occurs on a semiconductor surface and the chemisorbed species is charged, electrons are withdrawn from, or holes donated to, the solid if a negatively charged adsorbed species is formed. The result is a positive space charge extending into the solid and the energetics of this can be shown using energy band diagrams. A simple example of adsorption on an n-type semiconductor is shown in Fig. 19. The field in the space charge region moves electrons to the bulk and holes towards the surface. The bands are then bent and the majority charge carriers are depleted from the surface region. As a consequence the removal of further electrons is hindered and the (electronic) conductivity of the surface region is decreased in the case illustrated in the figure. Another consequence is that, due to the increasingly difficult removal of electrons, the original adsorption enthalpy becomes less negative and finally reaches zero. Of course this is only one, very simple example, and for a more detailed and comprehensive treatment we must refer the reader to the literature; see e.g. Ref. 131.

The electric field near to a surface directly influences the work function, i.e. the energy needed to remove an electron from a solid and the enthalpy of adsorption. This subject has recently been reviewed by Nowotny and Sloma [132].

As a consequence of the participation of the electrons (or electron holes) in the different defect equilibria discussed in Section 3.3, an important effect of space charges in oxides is the change in concentration of defects near the surface as discussed, e.g., by Maier [133]. As shown by that author this may also lead to a different dependence of defect concentrations and, for instance, conductivity on external parameters, such as the oxygen partial pressure, than is found in the bulk. This means that the equilibrium lines in the Brouwer diagrams of Figs. 2 and 3 have been shifted for the surface region of an oxide with respect to the position they have for the bulk. 


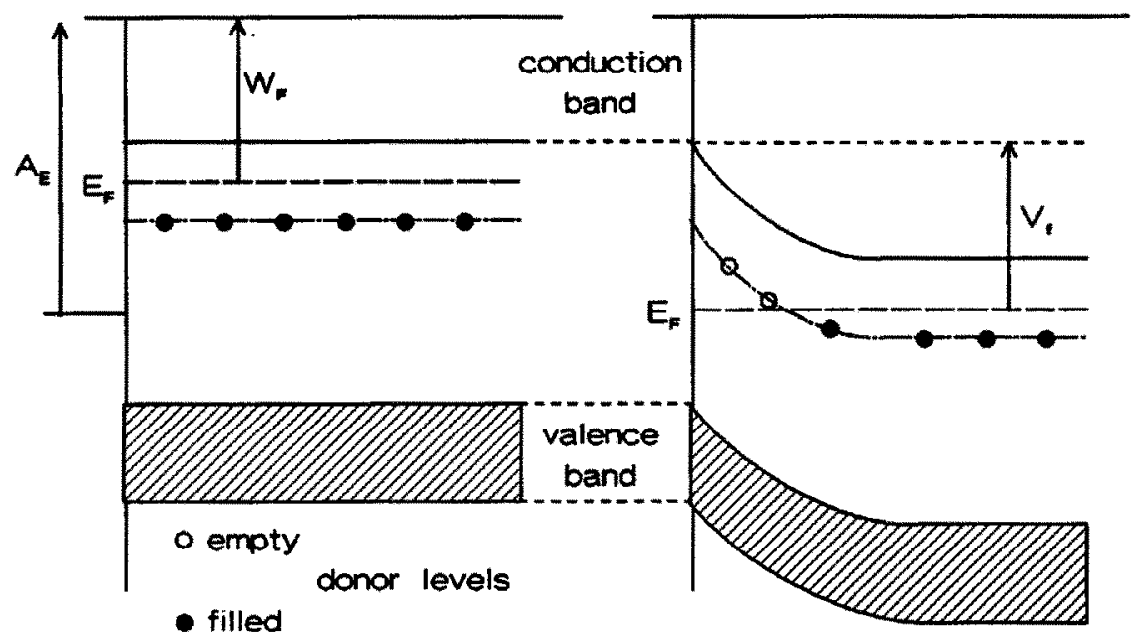

a)

b)

Fig. 19. Diagram of adsorption on an n-type semi-conductor: (a) before, (b) after chemisorption. $A_{E}=$ electron affinity of adsorbent, $E_{F}=$ Fermi level, $W_{F}=$ work function, $V_{f}=$ barrier height.

The electric field which arises due to the space charge also influences the transport properties of charged defects, such as the diffusion rate, which has been indicated, among others, by Nowotny [118]. Depending on the sign of the charge they are either accelerated or retarded by the internal field due to the space charge near the surface. In a series of papers Maier [134, 135, 136, 137] has discussed the enhanced transport properties, in particular the ion conductivity, by surface induced effects in heterogeneous systems near the interfaces between different phases or crystallites. It is shown, that these effects are due to the space charges and the accompanying electric fields.

Applications of these and similar considerations to charge transfer reactions at interfaces and to models for and development of chemical sensors have been reviewed by Göpel [138, 139]. It is unfortunate that there have been, as far as the present authors are aware, as yet no detailed applications of these ideas to the ion and mixed conducting oxides which form the subject matter of this review. 


\section{ADSORPTION AND SURFACE INTERACTION}

\subsection{General}

Many reviews are available covering adsorption on oxidic materials in general and on the type of oxides considered in this review in particular, e.g. those by Cimino and Carrà [14], Bielanski and Haber [31, 140], Che and Tench [141, 142], de Montgolfier [29], Claudel [13, 30] and Morrison [131], to mention only a few. For this reason only limited attention is paid to the many publications in the field of adsorption phenomena on oxides and for a more comprehensive study of these phenomena we refer the reader to the above-mentioned extensive reviews. Only some of the points needed to obtain a better insight into the subject treated in this review are considered in more detail.

Bielanski and Haber [31, 140] have discussed the role of oxygen in catalysis and in particular have treated the many different forms of adsorbed oxygen occurring on oxides. Fig. 20 (adapted from Ref. 31) gives a schematic survey of these forms and of their relative energies.

It is seen that the only negatively charged oxygen species which is stable in the gas phase with respect to $\mathrm{O}_{2}$ is the superoxide ion $\mathrm{O}_{2}{ }_{2}^{-}$. This has indeed often been observed as a commonly adsorbed oxygen species. All other negative oxygen species are unstable in the gas phase with respect to $\mathrm{O}_{2}$, but they will be stabilized by the Madelung energy as adsorbed species on the surface or in the bulk of solid oxides. In fact the $\mathrm{O}^{2-}$-ion is highly unstable in the gas phase, but is stabilized by the Madelung energy in the bulk of oxide crystal lattices and also, it is generally assumed, in their surface layers.

Bielanski and Haber have proposed a division of metal oxides into three main groups on the basis of their interaction with gaseous $\mathrm{O}_{2}$ :

1. p-type semiconductors, e.g., $\mathrm{NiO}, \mathrm{MnO}$, etc., which form electron-rich adsorbed species: $\mathrm{O}^{-}, \mathrm{O}^{2-}$;

2. a group consisting of n-type semiconductors, e.g., $\mathrm{ZnO}, \mathrm{TiO}_{2}, \mathrm{~V}_{2} \mathrm{O}_{5}$, etc. and dilute solutions of transition metal ions in isolators, e.g., $\mathrm{FeO}$ in $\mathrm{CaO}$, which form electron-poor, i.e. electrophilic, adsorbed species: $\mathrm{O}_{2}^{-}, \mathrm{O}^{-}$;

3. binary oxides in which lattice oxygen is present as $\mathrm{O}^{2-}$ in well-defined oxyanions, e.g., $\mathrm{Bi}_{2} \mathrm{O}_{3} \cdot \mathrm{MoO}_{3}$, on which no adsorbed oxygen species are formed but only $\mathrm{O}^{2-}$-anions, which are nucleophilic.

This correlates with some aspects of catalytic behaviour: oxides of group 1 tend to promote complete combustion of organic molecules, while type 2 and in particular type 3 lead to selective oxidation of hydrocarbons.

Che and Tench, in two extensive reviews, have treated the characterization and reactivity of mononuclear [141] and of molecular [142] oxygen species on oxide surfaces. These authors [142] remark that the abovementioned division of oxides into three 


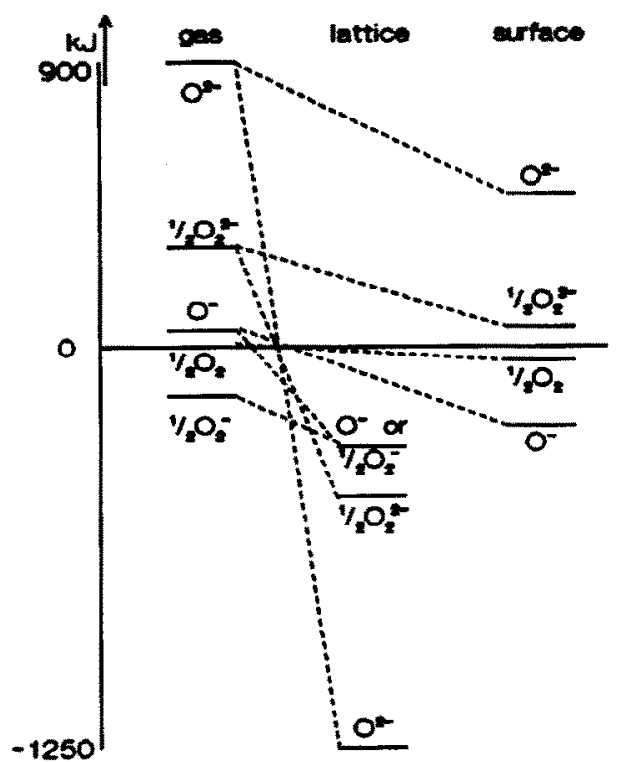

Fig. 20. Oxygen species occurring in catalysis [31]. The figure is schematic and the energies are not exactly to scale. groups seems to be an oversimplification, since it is probable that a range of adsorbed oxygen species can be observed on all these oxides given the right conditions. Of course the thermal stability of these species is likely to vary considerably.

In oxidation reactions occurring on these oxides oxygen can be involved in three distinct ways, more than one of which may be operative in any reaction mechanism:

1. a hydrogen or proton can be extracted from an adsorbed organic molecule giving a radical or carbanion;

2. attack on the organic molecule by a negative oxygen species whether adsorbed or lattice oxygen;

3. reoxidation of the catalyst with the formation of lattice oxygen used in a direct oxidation reaction.

Lamotte et al. [143] have studied, using infrared spectroscopy, the adsorption of $\mathrm{H}_{2}$ and $\mathrm{CO}$ on $\mathrm{ThO}_{2}$, in connection with its catalytic activity. Several different reversibly and irreversibly adsorbed $\mathrm{H}_{2}$ and $\mathrm{CO}$ species were found, but no connection with the conduction properties of the activated $\mathrm{ThO}_{2}$ is discussed.

The application of infrared spectroscopy in the study of oxygen adsorption on oxides has been discussed by Che et al. [144]. For end-on adsorbed dioxygen there is a clear correlation between the infrared absorption frequency and the bond order, which itself is directly related with the charge of the oxygen species: $\mathrm{O}_{2}^{+}: \mathrm{n}=1800-1850 \mathrm{~cm}^{-1}$, bond order $2.5, \mathrm{O}_{2}: \mathrm{n}=1550-1600 \mathrm{~cm}^{-1}$, bond order $2, \mathrm{O}_{2}: \mathrm{n}=1150-1200 \mathrm{~cm}^{-1}$, bond order 1.5 and $\mathrm{O}_{2}^{2-:}: \mathrm{n}=750-800 \mathrm{~cm}^{-1}$, bond order 1 . For side-on adsorbed oxygen the infrared absorption frequency is not as clear-cut due to opposing influences of $\sigma$ and $\pi$ bonding.

Takasu et al. $[145,146,147]$ studied the temperature programmed desorption of oxygen from nonstoichiometric praseodymium oxide and terbium oxide. Several different types of oxygen were found in the form of a number of desorption peaks at different temperatures. For $\mathrm{PrO}_{2-\mathrm{x}} \alpha, \beta, \gamma$ and $\delta$ oxygen desorption peaks were observed, depending mainly on the temperature of the oxygen pretreatment of the 
sample, $\alpha$ oxygen occurring at the lowest and $\delta$ oxygen at the highest temperature. For $\mathrm{TbO}_{2-\mathrm{x}}$ only $\alpha$ and $\beta$ oxygen desorption peaks were observed. Of these the $\alpha$ oxygen seems to be the most active and is involved in the oxidation of NO when this oxide is used as catalyst. XPS experiments have shown the presence of two different types of oxygen in the surface layer, in agreement with the thermal desorption results. That in these desorption processes lattice oxygen, and not only adsorbed or surface oxygen, is involved is clear from the amounts of oxygen which are involved: $7.5 \%$ in the case of $\mathrm{Pr}_{6} \mathrm{O}_{11}$ and $17 \%$ in the case of $\mathrm{TbO}_{1.81}$.

The influence of additives on the reduction and oxidation of terbium oxides was studied by Otsuka and Nakajima [148] who showed that the addition of the oxides of iron, cobalt and nickel increased the rates of oxidation and reduction. This was ascribed by the authors to an enhanced reduction (desorption) and oxidation (adsorption) due to an increased mobility of oxygen ions in the lattice.

\section{$8.20 x y g e n$ sorption and defect structure}

The sorption of oxygen by oxides is certainly expected to be influenced by their defect structure. This has for example, been studied by Zhang et al. [149] for perovskite-type oxides with the general formula $\mathrm{La}_{1-x} \mathrm{Sr}_{x} \mathrm{Co}_{1-y} \mathrm{Fe}_{y} \mathrm{O}_{3}$, using a temperature programmed desorption technique. The oxides with $0.2<\mathrm{x}<1.0$ show a desorption maximum between 150 and $250^{\circ} \mathrm{C}$ and this increases with increasing $x$. The oxygen desorbing at these low temperatures is called $\alpha$ oxygen. The amount of oxygen which desorbs is much larger than a monolayer and is roughly 0.3 to 0.4 of the molar amount of strontium present. There was not a significant change in the amount of desorption as a function of $y$, but the position of the peak maximum shifted to a minimum of about 40 ${ }^{\circ} \mathrm{C}$ (onset) and $150{ }^{\circ} \mathrm{C}$ maximum for $\mathrm{y}=0.6$. In agreement with earlier work [150] it is proposed that upon heating in vacuum or in a helium atmosphere oxygen desorbs and in this way charge compensation for the presence of strontium is achieved. Upon exposure to oxygen the oxygen vacancies become occupied by absorbed oxygen, while $\mathrm{Co}^{4+}$ ions are formed simultaneously.

If $\alpha$ oxygen is indeed associated with the oxygen vacancies due to the charge compensation caused by strontium doping, the $\mathrm{O} / \mathrm{Sr}$ ratio can lie between 0 and 0.5 , in good agreement with the experimental results.

The effect of iron substitution on the desorption of oxygen is probably related to the $\mathrm{Fe}^{3+}-\mathrm{Fe}^{4+}$ and $\mathrm{Co}^{3+}-\mathrm{Co}^{4+}$ charge equilibria. Another influence may be the crystal structure of these compounds. In the $\mathrm{La}_{1-\mathrm{x}} \mathrm{Sr}_{\mathrm{x}} \mathrm{CoO}_{3}$ system the structure changes above about $x=0.4$ where, due to ordering of oxygen vacancies, a two-phase mixture of perovskite and brownmillerite type structures is formed. In the iron containing perovskite, on the other hand, the perovskite structure is stable up to $x=1.0$. This means that the iron-ions, present on part of the B-sites, evidently suppress the ordering of oxygen 
vacancies, stabilizing the perovskite structure up to $x=1.0$.

Similar results were obtained by Teraoka et al. [151] in the study of a large number of perovskites of the types: $\mathrm{LaMO}_{3}(\mathrm{M}=\mathrm{Cr}, \mathrm{Mn}, \mathrm{Fe}, \mathrm{Co}, \mathrm{Ni}), \mathrm{La}_{1-\mathrm{x}} \mathrm{Sr}_{\mathrm{x}} \mathrm{O}_{3-\delta}(\mathrm{x}$ $=0,0.2,0.4,1), \mathrm{La}_{0.8} \mathrm{Sr}_{0.2} \mathrm{MO}_{3 \pm \delta}(\mathrm{M}=\mathrm{Mn}, \mathrm{Fe}, \mathrm{Co})$ and $\mathrm{La}_{0.8} \mathrm{~A}_{0.2} \mathrm{CoO}_{3 . \delta}(\mathrm{A}=\mathrm{Na}, \mathrm{Ca}$, $\mathrm{Sr}, \mathrm{Ba}$ ). In these compounds too the desorption of the two types of oxygen ( $\alpha$ and $\beta$ ) were observed. The $\alpha$ oxygen is accommodated in the oxygen vacancies which are formed due to the partial substitution of $\mathrm{La}$ by the A-type cations. The $\beta$ oxygen is connected with the reduction of the B-cations to a lower valency. 


\section{OXYGEN EXCHANGE}

\subsection{Introduction}

The exchange of oxygen between oxide surfaces and the gas phase has long been recognized as a multistep process, involving a sequence of transfer from the gas to the adsorbed phase (or vice versa), surface diffusion, reactions in the adsorbed phase and exchange of atoms. The detailed kinetics are considered to be important in elucidating the mechanism of heterogeneous oxidation catalysis. An attempt is made here to summarize the variety of processes that may determine the rate of overall oxygen exchange, the role of surface processes and bulk diffusion being emphasized. The oxygen isotope exchange method, as well as chemical relaxation techniques to study equilibration kinetics of oxides, are briefly discussed. Isotopic exchange is performed under conditions of equilibrium. In the transient method, the chemical relaxation of the oxide is studied after perturbation of the equilibrium state. These methods complement each other and it is believed that their combined application, whenever possible in conjoint use with spectroscopic techniques, such as FTIR, UV and EPR, has a major capability for the screening of the kinetics of oxygen exchange.

\subsection{Factors determining the rate of oxygen equilibration}

In general the rate of equilibration of oxides may be determined by any of the sequential steps shown in Fig. 21.

Steps (1) and (9) are diffusion controlled processes and are not influenced by the oxide proper. The activation enthalpy is low, usually $20-30$ $\mathrm{kJ} /$ mole. The importance of external diffusion through the gas phase for the overall kinetics can easily be checked by changing the linear flow rate of the gas which is led over the oxide surface, but is left out of consideration in the following.

Steps (2), (3), (7) and (8) may even take place at room temperature. Several species can occur as intermediates for the

\section{Oxygen Exchange}

steps in mechanism

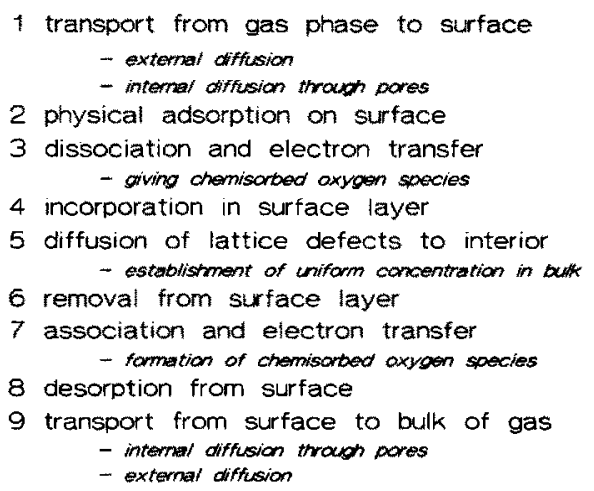

Fig. 21. Steps in oxygen exchange process. reduction of molecular oxygen, e.g.:

$\mathrm{O}_{2} \rightarrow \mathrm{O}_{2, \text { ads }} \rightarrow \mathrm{O}_{2 \text {,ads }}^{-} \rightarrow \mathrm{O}_{2 \text {,ads }}^{2-} \rightarrow \mathrm{O}_{\text {ads }}^{-} \rightarrow \mathrm{O}_{\text {lattice }}^{2-}$

or alternative routes. Among various possible intermediate ions, only the superoxide ion, 
$\mathrm{O}_{2}$; is stable with respect to gaseous $\mathrm{O}_{2}$ has already been mentioned in Section 8.1. It is therefore hardly surprising that the $\mathrm{O}_{2}^{-}$ads species happens to be the most commonly reported adsorbed dioxygen species [141]. All other species are unstable in the gas phase though their sorption on oxide surfaces may be stabilized by coulombic interactions. The $\mathrm{O}^{2-}$ ions are highly stabilized in the crystal field of the lattice, as was also indicated in Section 8.1. Their formation is considered to be equivalent to the incorporation in the oxide lattice (step (4)). Step (5), involving the joint transport of $\mathrm{O}^{2-}$ ions, and consequently of lattice defects, and electrons or electron holes, depending on the nature of the defects formed, usually proceeds only at high temperatures. Steps (2) - (4) and (6) - (8) may give rise to surface control proper, step (5) leads to bulk control of the exchange kinetics. In many cases a mixed type of rate control is observed, some examples of which are given below.

\subsection{Relaxation methods}

Data on the kinetics of equilibration (redox kinetics) of an oxide enable the calculation of the chemical diffusivity provided that the reaction at the gas-oxide interface either does not play an essential role in the overall equilibration kinetics or can be accounted for by an appropriate rate equation [152, 153, 154].

The (re)equilibration may follow after an instantaneous change of, for instance, the oxygen activity in the gas phase and involves the propagation of a composition gradient through a thin slab or single crystal of the oxide. The change in composition brings about a change in weight which can be monitored experimentally during the equilibration towards a new stoichiometric composition. The induced fluxes of ions and electrons, or corresponding lattice defects, compensate each other electrically as required by local charge neutrality. The slowest moving species determines the net rate of reequilibration. The effective diffusion coefficient, $D_{c}$, sometimes called the 'chemical' or 'ambipolar' diffusion coefficient, can be evaluated from the time dependence of equilibration using the appropriate solution to Fick's second diffusion law. This method is applicable if neither the diffusivity of the charge carriers nor the degree of ionization of lattice defects are functions of the composition change over the range in which this changes. By and large the chemical diffusivities are a function of the oxygen pressure, except for simple defect models [155], implying that values so obtained are averaged over the composition interval. Besides, the diffusivities obtained are apparent values and may involve possible contributions from diffusion along grain boundaries and dislocations.

Though weight measurements do not require any assumptions regarding the mechanism of oxygen uptake or release and therefore are to be preferred if possible, experiments can also be carried out if direct conversion to the change in stoichiometry is possible by measurement of, for example, the electrical conductivity, the movement of a colour front or of a p-n junction into a crystal (for references see, e.g., the paper by 
Childs et al. [153]). Bayoglu and Lorenzelli [156] used dilatometry as well as thermogravimetry to study the redox kinetics of a number of fluorite type oxides. It is noteworthy that, for example, a temperature gradient $[157,158]$ can also provide a driving force for chemical diffusion.

If an oxide specimen is subjected to a fixed oxygen potential gradient oxygen will permeate through the specimen from the high $\mathrm{P}_{\mathrm{O} 2}$ to the low $\mathrm{PO}_{\mathrm{O} 2}$ side. This forms the basis of the so-called 'time-lag' method in which the transient process towards steadystate oxygen permeation is monitored [159, 160]. The chemical diffusivity is calculated from Fick's second law of diffusion, which is solved under the special initial and boundary conditions applicable to this type of experiment.

To simplify the calculations it is commonly assumed that the surface of the sample equilibrates immediately with the newly imposed atmosphere [152, 153,154,161, 162], which leads to well-known standard analytical solutions of the diffusion equation [163]. However, for oxides like, for example, $\mathrm{Fe}_{1-\mathrm{x}} \mathrm{O}[164,165]$ and $\mathrm{Mn}_{1-\mathrm{x}} \mathrm{O}$ [166] it has been observed that the surface reaction exerts a partial control over the overall reequilibration kinetics. Surface controlled kinetics are inevitably encountered upon decreasing the sample dimensions. Numerical methods for the analysis of the experimental data in the case of a partial control of the reequilibration kinetics by the surface reaction, invoking different type of rate laws for the surface reaction, have been proposed by Neuman [167] and by Gesmundo and co-workers [168, 169, 170]. A competition between surface processes and diffusion of lattice defects has also been assumed to explain data of steady-state oxygen permeation through calcia-stabilized zirconia [171] and erbia-stabilized bismuth oxide [172], which is discussed in more detail in Section 10 of this paper.

The importance of the surface reaction on the reequilibration kinetics of nonstoichiometric oxides has been examined by Nowotny and Wagner et al. [173, $174,175,176]$, emphasizing the possible role of segregation of lattice components toward the surface. A large number of kinetic studies are considered in which the data from relaxation measurements appear to exhibit mixed control, i.e. the overall kinetics are determined by both surface reactions and bulk diffusion [173]. A model has been proposed in which the transport of lattice defects from the surface to the bulk (and vice versa), and hence the rate of equilibration, is affected by the electrical barrier across the junction between the crystalline bulk and a quasi-isolated segregated surface layer $[174,175,176]$.

Rather than using gas-solid exchange to impose oxygen activities at the boundaries and changes therein, solid state electrochemical methods can also be applied for this purpose $[177,178,179,180]$. Electrochemical techniques are in particular attractive because they allow an easy combination of transient and equilibrium measurements. An extensive review dealing with the use of galvanic cells for kinetic 
studies has been published by Weppner and Huggins [181]. Either the current through the cell (potentiostatic mode) or the cell voltage (galvanostatic mode) is the monitored variable during the relaxation process. Chemical diffusivities in oxides so obtained have been published for example, for: doped $\mathrm{ZrO}_{2}$ and $\mathrm{ThO}_{2}[182,183,184]$, for $\mathrm{Fe}_{1-\mathrm{x}} \mathrm{O}$ [185] and for $\mathrm{La}_{0.79} \mathrm{Sr}_{0.20} \mathrm{MnO}_{3-\mathrm{x}}[186]$.

If the range over which the nonstoichiometry can change by changing $\mathrm{P}_{\mathrm{O} 2}$ is large enough, with a concomitant change in conductivity, and if equilibration is sufficiently fast, the material can be used to construct a gas sensor [187]. A number of perovskite-type oxides, such as the cobaltates, $\mathrm{Ln}_{1-x} \mathrm{M}_{\mathrm{x}} \mathrm{CoO}_{3-\delta}$, and nickelites, $\mathrm{Ln}_{1-\mathrm{x}} \mathrm{M}_{\mathrm{x}} \mathrm{NiO}_{3+\delta}$ (where $\mathrm{Ln}$ $=$ lanthanide and $\mathbf{M}=$ alkaline earth), satisfies these requirements as discussed by Kudo [188]. The thickness of the sensing oxide layer usually is $10-20 \mu \mathrm{m}$, being printed as a paste, obtained from mixing fine powder with an oily ingredient, on an alumina substrate. These sensors not only respond to oxygen, but also to alcohols and to reducing gases. The reactions at the oxide surface are likely to proceed via the oxidation-reduction model (see Section 11.3), since these perovskite-type oxides are known to be good oxidation catalysts. The sensing principle differs from that of thick film tin oxide $\left(\mathrm{SnO}_{2}\right)$ gas sensors, where the change in conductivity (or resistivity) is caused by a change in the potential barrier at neck-shaped grain boundaries due to chemisorbed oxygen [189].

Similar to the chemical relaxation method, transient data from direct ${ }^{18} \mathrm{O} \cdot{ }^{16} \mathrm{O}$ exchange discussed in the next section enable calculation of the tracer diffusivity and the surface oxygen exchange rate. Both can be obtained from the change in the ${ }^{18} \mathrm{O} /{ }^{16} \mathrm{O}$ concentration ratio in the gas phase upon exchange in a fixed volume of ${ }^{18} \mathrm{O}_{2}$-enriched oxygen as measured with a mass spectrometer $[190,191]$, weight measurements [192], depth concentration profile measurements using secondary ion mass spectroscopy (SIMS) after exchange for a fixed period of time [193, 194], or from combined methods $[195,196,197]$. The interrelation between diffusion coefficient, mobility and conductivity has been discussed in Section 3.5. Besides ${ }^{18} \mathrm{O}_{2}$ and ${ }^{16} \mathrm{O}_{2}$ molecules ${ }^{18} \mathrm{O}^{16} \mathrm{O}$ molecules will also be formed in the gas phase. The time dependences of the concentrations of each these species are considered to be highly indicative of the steps which occur sequentially during oxygen exchange, i.e. adsorption, dissociation, etc.

Relaxation techniques applied to heterogeneous reactions have been reviewed by Parravano [198].

\subsection{Oxygen isotopic exchange}

\subsubsection{Types of exchange}

The kinetics of the oxide-catalyzed homomolecular isotopic exchange reaction of oxygen in the gas phase: 


$$
{ }^{18} \mathrm{O}_{2}+{ }^{16} \mathrm{O}_{2}=2{ }^{18} \mathrm{O}{ }^{16} \mathrm{O}
$$

and the accompanying direct exchange (heterogeneous exchange) of gaseous ${ }^{18} \mathrm{O}_{2}$ with lattice oxygen has been studied extensively. The principal aim of many of these studies has been to establish correlations between the rates of oxygen exchange reactions on oxides and their behaviour as oxidation catalysts for various reactants. At the present time there are abundant experimental data available in the literature on the oxygen isotopic exchange of oxides (powders) and metals with sorbed oxygen, presented in several reviews [199, 200, 201, 202, 203, 204, 205].

Data on oxygen isotopic exchange permit direct conclusions to be drawn about the nature of the surface oxygen bond. Full analysis of experimental data, however, is only simple if the surface oxide layer is homogeneous relative to the isotopic exchange process, which is considered to be the case if (a) surface oxygen exchanges much more rapidly with the gas phase than with lattice oxygen or if (b) self diffusion of oxygen in the oxide proceeds much more rapidly than the heterogeneous exchange rate. Kinetic isotope effects are assumed to be absent.

Measurements are performed, preferably as a function of temperature and oxygen partial pressure, using a mass spectrometer for oxygen isotopic analysis of the $\left({ }^{18} \mathrm{O}_{2}\right.$ enriched) gas phase. For detailed descriptions of experimental conditions and equipment we refer the reader to the literature [202, 206, 207].

The kinetics of isotope exchange reactions have been derived on a statistical basis by Klier et al. [208] and Muzykantov et al. [209] and only the salient features are outlined here. First order kinetics are assumed, which is in agreement with observations made by McKay [210], irrespective of the real mechanism by which the exchange reaction proceeds. Though McKay's suggestion has been widely adopted in describing isotopic exchange reactions serious limitations arise if concentrations of intermediates cannot be neglected with respect to those of reactants and if the rate determining step cannot be specified, as discussed by Takaishi [211]. According to these statistical theories there are three elementary processes by which gaseous oxygen molecules can exchange their atoms in the presence of an oxide catalyst (and which can be distinguished experimentally). These are as follows.

(1) The oxygen molecule can exchange one of its atoms with the oxide at a rate $\mathbf{R}^{\prime}$. It is assumed [200] that oxygen is dissociatively adsorbed on the surface, on a local surface oxygen vacancy or $F$ centre, one atom being immediately incorporated, the other migrating over the surface until it has a recombinative encounter with another adsorbed atom, resulting in desorption as a molecule. If there is insufficient surface mobility of chemisorbed oxygen $R^{\prime}$ is expected to be low. Another proposed mechanism is an Eley-Rideal one, involving exchange via a triangular intermediate, $\left({ }^{18} \mathrm{O}^{18} \mathrm{O}^{16} \mathrm{O}\right)_{\text {ads }}$, between a molecule from the gas phase 
and a surface atom of oxygen [204, 205].

(2) The oxygen molecule can exchange both atoms with the oxide at a rate $R^{*}$. One oxygen molecule is adsorbed, another being desorbed at the same time [202, 204]. As is obvious, such a mechanism requires pairs or clusters of vacancies to be present at the oxide surface. Another mechanism, proposed by Bonhoeffer and Farkas [212], involves dissociative adsorption forming chemisorbed atoms, followed by fast exchange with lattice oxygen. The isotopic composition of the desorbed molecule is equal to that of the oxide surface. It has also been suggested that the reaction proceeds via a four-atom, $\left({ }^{18} \mathrm{O}^{18} \mathrm{O}^{16} \mathrm{O}^{16} \mathrm{O}\right)_{\text {ads }}$, transient complex [202].

(3) The oxygen molecule cannot exchange its atoms with the oxide, but only with atoms of other gas phase molecules at a rate $\mathbf{R}$, via an adsorbed intermediate. This process may be realized by reversible dissociative adsorption, with a rate which is much higher than that of the exchange with lattice oxygen [200 - 202], or by isotopic exchange via a known activated intermediate (Eley-Rideal mechanism) which is most favoured by spectroscopic studies [141, 142]. Since no lattice oxygens are involved, this reaction runs almost exclusively at low temperatures, where self diffusion of oxygen in the oxide is negligible.

If the exchange follows all indicated routes simultaneously the overall homomolecular exchange rate, i.e. the rate of redistribution of the isotopic molecular forms in the gas phase, is expressed by:

$\tau_{\text {homo }}=\mathbf{R}+\mathbf{R}^{\prime}+\mathbf{R}^{\prime \prime}$

and the rate of heterogeneous exchange, i.e. the rate of change of the isotopic composition of the solid oxide is:

$r_{\text {hetero }}=1 / 2 \mathbf{R}^{\prime}+\mathbf{R}^{\prime \prime}$

where the various rate constants are expressed as the number of oxygen molecules exchanging per unit time and area or in equivalent dimensions.

The heterogeneous exchange rate, $\mathrm{r}_{\text {hetero }}$ is the quantity that can be obtained most directly by experiment since it equals the rate of exponential decay of the heavy isotopic concentration in the gas phase. The value of the homomolecular exchange rate, $\mathrm{r}_{\text {homo, }}$, can be deduced from the decay rate of concentrations of molecules with masses 32 $\left({ }^{16} \mathrm{O}_{2}\right), 34\left({ }^{16} \mathrm{O}^{18} \mathrm{O}\right)$ and $36\left({ }^{18} \mathrm{O}_{2}\right)$. This is only possible if a non-equilibrium gas mixture for which the concentration function $F_{\mathfrak{c}}$, defined by

$$
F_{c}=\frac{\left[^{16} \mathrm{O}^{18} \mathrm{O}\right]^{2}}{\left[{ }^{16} \mathrm{O}_{2}\right]\left[{ }^{18} \mathrm{O}_{2}\right]}
$$


is not equal to the equilibrium constant of reaction (31), $K=4$. When such a gas mixture is subjected to exchange, and provided that the oxide has the same isotopic composition as the gas phase, $r_{\text {homo }}$ can be obtained. The three rates of exchange, $R, R^{\prime}$ and $R^{\prime \prime}$, can be obtained from one experiment by simultaneous measurement of the concentrations of all individual isotopic species during the course of the experiment.

\subsubsection{Some experimental observations}

Oxides of the rare earth metals show a high activity for both types of oxygen exchange $[213,214,215]$. In particular on those oxides which show nonstoichiometry, e.g. oxides of $\mathrm{Pr}, \mathrm{Ce}$ and $\mathrm{Tb}$, sharp maxima in both exchange rates are observed. This is consistent with the ease of the $\mathrm{Ln}^{3+}=\mathrm{Ln}^{4+}$ transition and with the correspondingly high oxygen mobility. Although, in the range of 450 to $750 \mathrm{~K}$ activation enthalpies and exchange rates of the various oxides vary widely, the rate of exchange in molecular oxygen $\left(r_{\text {homo }}\right)$ is almost always equal to the rate of isotopic exchange with oxide oxygen $\left(\mathrm{r}_{\text {hetero }}\right)$.

The equality of homomolecular and heterogeneous exchange rates reflects a general trend of oxides showing a high degree of oxygen mobility. Apart from the lanthanide oxides this behaviour is observed for, e.g., $\mathrm{V}_{2} \mathrm{O}_{5}, \mathrm{Cr}_{2} \mathrm{O}_{3}, \mathrm{ZnO}, \mathrm{Fe}_{2} \mathrm{O}_{3}, \mathrm{MoO}_{3}$, $\mathrm{WO}_{3}, \mathrm{PbO}, \mathrm{Bi}_{2} \mathrm{O}_{3}[199$ - 205]. Their surface layers remain homogeneous towards isotopic exchange because the rate of self diffusion of oxygen in the oxide is much faster than that of heterogeneous exchange. The kinetics of isotopic exchange are exponential up to the point of full isotopic equilibrium between the gas phase and the oxide.

For other oxides, e.g. $\mathrm{NiO}, \mathrm{Co}_{3} \mathrm{O}_{4}, \mathrm{MnO}_{2}$ and $\mathrm{CuO}$, the heterogeneous exchange rate decreases sharply with the extent of exchange. The exchange progresses only up to a fractional value of the total oxygen content ('depth of exchange'), which is usually restricted to one or two monolayers of the oxide [203]. Often the initial sharp decrease is followed by a slow one, indicating slow oxygen self diffusion. In these cases the equation for diffusion must be invoked to describe the overall exchange kinetics [216]. Mathematical descriptions considering surface oxygen as a discrete inhomogeneity with respect to its reactivity and bond strength, can be found in $[217,218,219]$. The idea behind these is that many calorimetric, thermodesorption and pulsed kinetic results illustrate that the reactivity and strength of oxygen bonding of oxide catalysts depend to a noticeable extent on the oxygen coverage of the oxidic surface.

For nearly all oxides whose surfaces are inhomogeneous towards isotopic exchange, except for $\gamma-\mathrm{Al}_{2} \mathrm{O}_{3}$, it is found that the initial rates of homomolecular and heterogeneous exchange are in close agreement $[200,201]$. Thus it seems that the isotopic exchange in molecular oxygen (reaction (31)) always proceeds with the participation of oxide surface oxygen. 
The low-temperature activity to homomolecular exchange is increased to a marked degree, although it is unstable with time, if oxides are partly reduced by heating in vacuum [200, 201]. On, $\mathrm{ZnO}$, for instance, the reaction proceeds even at liquid nitrogen temperature [220]. The activity returns to its original value after heat treatment in oxygen. This behaviour is generally seen, except for oxides with a high oxygen mobility $\left(\mathrm{Bi}_{2} \mathrm{O}_{3}, \mathrm{~V}_{2} \mathrm{O}_{5}, \mathrm{MoO}_{3}\right)$. The observed phenomenon is attributed to the creation of local surface defects (oxygen vacancies, interstitial metal atoms) by the vacuum ageing procedure. High temperature and sufficient oxygen pressure are required for restoration of the lattice at the surface. Note that similar defects will be produced by chemical reduction of the oxide.

9.4.3 Stepwise mechanism and the type of oxygen species involved in the surface exchange reaction

If we note that the detailed mechanism of the surface exchange reaction is irrelevant in deriving the kinetic expressions on a statistical basis, a consecutive mechanism may be considered in which the rates of the various processes, $\mathbf{R}, \mathbf{R}^{\prime}$ and $\mathbf{R}^{\prime \prime}$ are determined by the detailed balance of successive steps [221]. The simplest consecutive mechanism is to describe oxygen exchange as a sequence of two reaction steps. This has recently been applied to describe oxygen isotopic exchange of 25 mole \% erbia-stabilized bismuth oxide (BE25) [206, 222, 223]. An example is given in Fig. 22 , showing the time dependences of the relative amounts of mass components $32\left({ }^{16} \mathrm{O}_{2}\right)$, $34\left({ }^{16} \mathrm{O}^{18} \mathrm{O}\right)$ and $36\left({ }^{18} \mathrm{O}_{2}\right)$ in the gas phase during exchange on $\mathrm{BE} 25$ powder at $873 \mathrm{~K}$ together with simulated results. In our laboratory a number of oxides are currently under investigation to check the validity of models such as the one proposed for BE25 and others [224].

Measurements indicate that the dissociative adsorption of oxygen is the rate determining step in surface oxygen exchange on BE25 [206, 222]. The reaction sequence may be written as:

$$
\begin{aligned}
& \mathrm{O}_{2} \rightleftharpoons 2 \mathrm{O}_{\mathrm{ads}} \\
& \mathrm{O}_{\mathrm{ads}}+\mathrm{V}_{\mathrm{O}}^{\infty}=\mathrm{O}_{\mathrm{O}}^{\mathrm{x}}+2 \mathrm{~h}^{\bullet}
\end{aligned}
$$

or equivalently, noting that the charge of the adsorbed species is assumed to be irrelevant in describing the exchange proces (in many cases definite information is not available). 


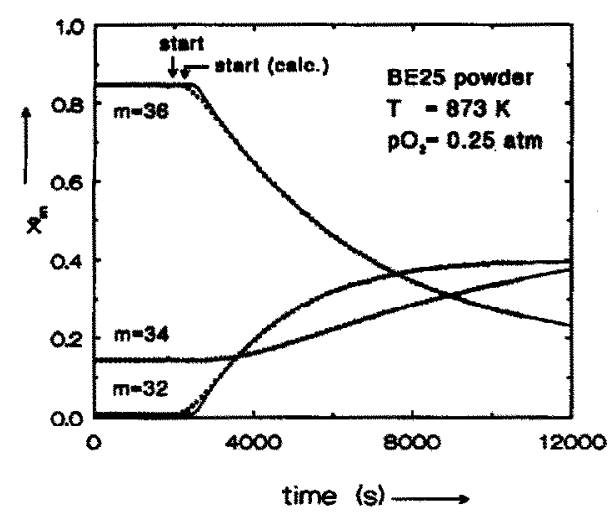

Fig. 22. Relative amounts, $x_{m}$, of oxygen isotopic components in the gas phase as a function of time during exchange of BE25 at $873 \mathrm{~K}$. Full lines denote simulated results [222].

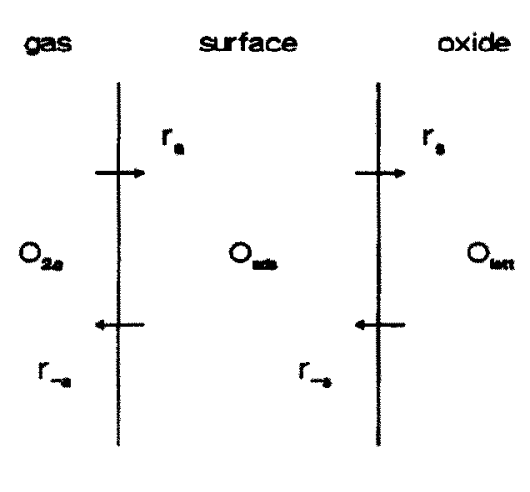

Fig. 23. Schematic representation of twostep oxygen exchange mechanism.

The main feature of the two-step model depicted in Fig. 23 is to describe oxygen exchange as a dynamic process in which the concentrations of isotopic species at the oxide surface are balanced by two reversible rate processes. For BE25 these are the rate of adsorption of oxygen, $r_{a}$, and that of its subsequent incorporation (and diffusion) into the lattice, $r_{s}$. During its residence time each oxygen ad-atom has an independent chance $p$, to exchange with lattice oxygen before recombination with another ad-atom before being desorbed as a molecule. The corresponding chance that no exchange with lattice oxygen occurs is $(1-p)$. With the rate of adsorption/desorption equal to $r_{\mathfrak{a}}$, one finds:

$$
\begin{aligned}
& \mathbf{R}=r_{a} \times(1-p)^{2} \\
& \mathbf{R}^{\prime}=r_{a} \times 2 p(1-p) \\
& R^{\prime \prime}=r_{a} \times p^{2}
\end{aligned}
$$

assuming no branching paths of the proposed mechanism. From Eqns. (32), (33) and (36), and reaction scheme (35) it is readily seen that: 
$\mathrm{p}=\frac{\mathrm{r}_{\mathrm{s}}}{\mathrm{r}_{\mathrm{a}}+\mathrm{r}_{\mathrm{s}}}$

$r_{\text {hetero }}=r_{a} \times p=\frac{r_{a} r_{s}}{r_{a}+r_{s}}$

$r_{\text {homo }}=r_{a}$

In the limit of the inequality $r_{a} \gg r_{s}$ one finds $p \approx 0$, hence in this case only the $R$ process occurs. If $r_{a}$ \& $r_{s}$ then $p \approx 1$ and only the $R$ " process occurs, while $r_{\text {hetero }} \approx$ $r_{\text {homo }}$. In all intermediate cases all processes are active.

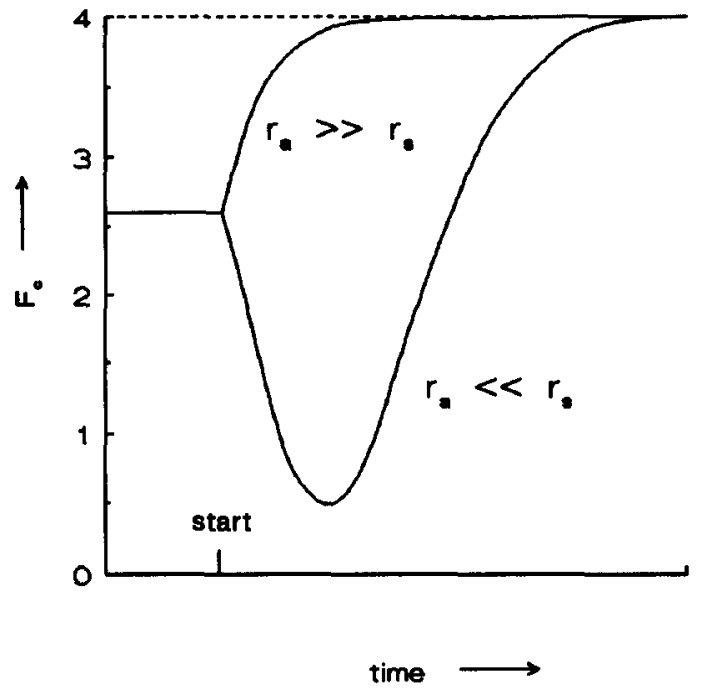

Fig. 24. Change of the concentration function $F_{c}$ (equation (34)) as a function of time for two limiting cases.
The equilibrium constant of reaction (31) equals 4. Fig. 24 illustrates that if a gas mixture which is close to equilibrium is subjected to exchange the gas phase is immediately driven to the equilibrium state in case inequality $r_{a} \gg r_{s}$ holds. If, however, $r_{a} \ll r_{s}$, as is observed for BE25, the system moves away from equilibrium during the initial stage while equilibrium is reestablished in the final stage of the exchange process.

In general, a number of arguments support a stepwise mechanism of oxygen exchange. The close coincidence of the initial rates of

homomolecular and heterogeneous exchange indicates a common role of chemisorbed oxygen species in determining the rates of the two reactions. Moreover, for nearly all oxides processes $R^{\prime}$ and $R^{\prime \prime}$ occur simultaneously, the extent to which they vary being a function of temperature and oxygen pressure. Of these processes $\mathbf{R}^{\prime}$ is the one which is most commonly observed, process $\mathbf{R}^{\prime \prime}$ being the one preferred at higher temperatures. A few exceptions are, e.g., $\mathrm{V}_{2} \mathrm{O}_{5}, \mathrm{MoO}_{3}, \mathrm{PbO}, \mathrm{Bi}_{2} \mathrm{O}_{3}$ and $\mathrm{WO}_{3}$, where a pure $\mathrm{R}^{\text {" }}$ mechanism has been reported (differing by at least an order of magnitude from $\mathbf{R}^{\prime}$ ). These solids are well characterized by their high oxygen mobility, which is further manifested in their ability to exchange all lattice oxygen.

On the evidence of spectroscopic studies an associative mechanism of oxygen 
exchange involving the direct participation of gas phase molecules via activated intermediates with chemisorbed species may prevail at low temperatures.

Using Fourier transform infrared spectroscopy (FTIR) $\mathrm{Li}$ et al. [225] arrive at the conclusion that isotopic exchange between gaseous $\mathrm{O}_{2}$ and lattice oxygen of $\mathrm{CeO}_{2}$ does not occur with a measurable rate at temperatures below $373 \mathrm{~K}$, but site and isotopic exchange reactions with adsorbed superoxide species, $\mathrm{O}_{2}{ }^{-}$ads, continue to proceed even at temperatures as low as $200 \mathrm{~K}$. The isotopic exchange reaction is presumed to involve a tetraoxygen intermediate, $\mathrm{O}_{4}^{-}$ads, as a result of the reaction of gaseous $\mathrm{O}_{2}$ with adsorbed superoxide species, as has been proposed in oxygen isotopic exchange over $\mathrm{MgO}, \mathrm{ZnO}$ and $\mathrm{TiO}_{2}$ from EPR measurements (electron paramagnetic resonance). For further references, see Ref. 225.

The presence of the $\mathrm{O}_{2}{ }^{-}$ads ion on oxide surfaces has been identified by EPR on numerous oxides and this is by far the most commonly reported dioxygen species. There is also extensive evidence for the ionosorption of $\mathrm{O}_{\text {ads }}^{-}$and (its possible intermediate after reaction with gaseous oxygen) $\mathrm{O}_{3}{ }^{-}$ads species as discussed in the reviews on the characterization and reactivity of mononuclear [141] and molecular [142] oxygen species on oxide surfaces by Che and Tench.

The superoxide ion may be considered as an intermediate in the formation of oxygen species more rich in electrons. On $\mathrm{SnO}_{2}$ films the equilibrium of the reaction

$$
\mathrm{O}_{2, \mathrm{ads}}^{-}+\mathrm{e}^{-}=2 \mathrm{O}_{\mathrm{ads}}^{-}
$$

shifts to the right with increasing temperature as concluded from EPR measurements [131]. Above about $450 \mathrm{~K} \mathrm{O}^{-}$ads is the prevailing species. Similar conclusions were drawn for $\mathrm{ZnO}$ from Hall effect measurements [226]. An energy diagram of various oxygen species in the gas phase, adsorbed at the surface and bound within the oxide lattice is given in [31], see also Section 8, Fig. 20.

\subsubsection{Surface oxygen exchange of ion and mixed conducting oxides}

Steele, Kilner and co-workers [193] were among the first to measure oxygen surface exchange on single crystal and dense, sintered polycrystalline samples of ion conducting oxides using combined ${ }^{18} \mathrm{O}-{ }^{16} \mathrm{O}$ isotopic exchange and secondary ion mass spectroscopy (SIMS). The oxygen surface exchange rate and (tracer) diffusivity which can be derived from these experiments govern the process of oxygen transport through electrochemical devices such as oxygen pumps, solid oxide fuel cells etc. Quite a few studies have been reported by these authors, of which a selected group is given in our reference list [227, 228, 229]. In a recent paper by Kurumchin and Perfil'ev [230] these authors present an overview of earlier work dealing with ${ }^{18} \mathrm{O}$ exchange for investigating of the influence of the gas phase, electrode and electrolytes based on $\mathrm{Bi}_{2} \mathrm{O}_{3}$, $\mathrm{CeO}_{2}$ and $\mathrm{ZrO}_{2}$ on the rate of the interfacial kinetics. Values of some experimentally 
determined oxygen surface exchange rates ( $\mathrm{r}_{\text {hetero }}$ ) of ion and mixed conducting oxides are given in Table 9.

The value measured for the (heterogeneous) exchange rate relates to the electrochemical accessible exchange current density, $i_{0}$, which expresses the balanced faradaic activity at equilibrium, assuming no activation on the applied electrodes. That is, the assumption is made that the electrodes are only acting as current collectors and do not influence the rate determining step in the overall surface exchange reaction. For $\mathrm{Bi}_{2} \mathrm{O}_{3}$ based oxide electrolytes good agreement was obtained between the values obtained from both methods, indicating that the dissociative adsorption of oxygen occurs predominantly on the electrolyte surface [228]. SIMS measurement revealed a rather flat diffusion profile, confirming that the surface reaction is rate-determining in the overall oxygen exchange. These observations have been verified by others $[206,222,230]$.

The $\mathrm{Bi}_{2} \mathrm{O}_{3}$ and $\mathrm{CeO}_{2}$ based electrolytes show a much larger activity in oxygen exchange with the gas phase than do the $\mathrm{ZrO}_{2}$ based electrolytes [228, 230]. This has been ascribed to the larger electronic conductivity of the former electrolytes, which is about one or two orders of magnitude higher than that in the stabilized zirconias [230]. In contrast to $\mathrm{Bi}_{2} \mathrm{O}_{3}$ based electrolytes, the electrode material $(\mathrm{Pt}, \mathrm{Ag})$ considerably enhances the exchange kinetics of $\mathrm{ZrO}_{2}$ based electrolytes. The catalytic effect depends on the amount of metal applied, as observed for $\mathrm{Pt}$, and therefore coincides with the total length of the three-phase boundary line (gas, electrode, electrolyte).

The type of molecules present in the gas phase also exerts a profound influence on the exchange kinetics. The isotopic exchange between the oxygen of carbon dioxide and lattice oxygen proceeds much more rapidly on yttria stabilized zirconia (YSZ) than the exchange with $\mathrm{O}_{2}[230,231]$. Park and Olander [192] studied the oxygen diffusivity in single-crystal tetragonal zirconia monitoring the weight increase due to the reaction:

$$
\mathrm{Zr}^{16} \mathrm{O}_{2}+2 \mathrm{H}_{2}^{18} \mathrm{O}=\mathrm{Zr}^{18} \mathrm{O}_{2}+2 \mathrm{H}_{2}^{16} \mathrm{O}
$$

The derived values of the surface exchange rate are many orders of magnitude larger than those measured by Steele et al. using ${ }^{18} \mathrm{O}_{2}$ as the source of oxygen [228].

Similar observations have been made on bismuth molybdate. On this catalyst neither the homomolecular nor the heterogeneous exchange rate in ${ }^{18} \mathrm{O}_{2}$-enriched oxygen can be observed experimentally [232]. On the other hand, Novakova and Jiru [233] have shown that the exchange of water with lattice oxygen on an industrial bismuth molybdate catalyst proceeds rapidly at $200^{\circ} \mathrm{C}$ and is even measurable at room temperature. 
Table 9

Oxygen surface exchange rates ( $\mathrm{r}_{\text {hetero }}$ ) of some ion and mixed conducting oxides

\begin{tabular}{|c|c|c|c|c|}
\hline Compound & $\begin{array}{l}\mathrm{r}_{\text {hetexo }} \\
\left(\mathrm{mol} \cdot \mathrm{m}^{-2} \cdot \mathrm{s}^{-1}\right)\end{array}$ & $\begin{array}{c}\mathrm{T} \\
\left({ }^{\circ} \mathrm{C}\right)\end{array}$ & method & Ref. \\
\hline $\mathrm{Bi}_{2} \mathrm{O}_{3}-25 \mathrm{~m} \% \mathrm{Er}_{2} \mathrm{O}_{3}$ & $\begin{array}{l}2.1 \times 10^{-5} \\
5.6 \times 10^{-4}(f) \\
1.2 \times 10^{-5} \\
4.4 \times 10^{-4} \\
\end{array}$ & $\begin{array}{l}600 \\
800 \\
800 \\
800 \\
\end{array}$ & $\begin{array}{l}\mathbf{a} \\
\mathbf{a} \\
\mathrm{c} \\
\mathrm{c}\end{array}$ & {$[222]$} \\
\hline $\mathrm{Bi}_{2} \mathrm{O}_{3}-40 \mathrm{~m} \% \mathrm{~Tb}_{2} \mathrm{O}_{3.5}$ & $\begin{array}{l}5.4 \times 10^{-5} \\
9.3 \times 10^{-4}(\mathrm{f}) \\
5.7 \times 10^{-5} \\
1.3 \times 10^{-3} \\
\end{array}$ & $\begin{array}{l}600 \\
800 \\
600 \\
800 \\
\end{array}$ & $\begin{array}{l}\mathrm{a} \\
\mathrm{a} \\
\mathrm{c} \\
\mathrm{c}\end{array}$ & {$[222]$} \\
\hline $\begin{array}{l}\mathrm{ZrO}_{2}-16 \mathrm{~m} \% \mathrm{Y}_{2} \mathrm{O}_{3} \text { (d) } \\
\mathrm{ZrO}_{2}-16 \mathrm{~m} \% \mathrm{Y}_{2} \mathrm{O}_{3} \text { (d) }\end{array}$ & $\begin{array}{l}2.8 \times 10^{-6} \\
1.5 \times 10^{-5} \\
\end{array}$ & $\begin{array}{l}700 \\
700 \\
\end{array}$ & $\begin{array}{l}\mathrm{b} \\
\mathrm{b} \\
\end{array}$ & [228] \\
\hline $\begin{array}{l}\mathrm{ZrO}_{2}-10 \mathrm{~m} \% \mathrm{Y}_{2} \mathrm{O}_{3} \\
\text { "with overlayer } \mathrm{PrO}_{2-\delta} \\
\text { with } \mathrm{Pt} \text { deposition }\end{array}$ & $\begin{array}{l}5.3 \times 10^{-9}(\mathrm{~g}) \\
2.6 \times 10^{-2}(\mathrm{~g}) \\
5.3 \times 10^{-7}(\mathrm{f}, \mathrm{g})\end{array}$ & $\begin{array}{l}600 \\
600 \\
600 \\
\end{array}$ & $\begin{array}{l}\mathbf{a} \\
\mathbf{a} \\
\mathbf{a}\end{array}$ & [230] \\
\hline $\mathrm{La}_{0.6} \mathrm{Sr}_{0.4} \mathrm{CoO}_{3}$ & $\begin{array}{l}2.3 \times 10^{-4} \\
6.4 \times 10^{-3} \\
\end{array}$ & $\begin{array}{l}600 \\
800 \\
\end{array}$ & $\begin{array}{l}\text { c } \\
\text { c }\end{array}$ & {$[239,240]$} \\
\hline $\mathrm{La}_{0.6} \mathrm{Sr}_{0.4} \mathrm{Co}_{0.98} \mathrm{Ni}_{0.02} \mathrm{O}_{3}$ & $\begin{array}{l}1.5 \times 10^{-3} \\
2.4 \times 10^{-2} \\
\end{array}$ & $\begin{array}{l}600 \\
800 \\
\end{array}$ & $\begin{array}{l}c \\
c\end{array}$ & {$[239,240]$} \\
\hline YSZ/Pt (e) & $\begin{array}{l}2.7 \times 10^{-6} \\
6.9 \times 10^{-4} \\
\end{array}$ & $\begin{array}{l}600 \\
800 \\
\end{array}$ & $\begin{array}{l}\text { c } \\
\text { c }\end{array}$ & {$[242]$} \\
\hline $\begin{array}{l}\mathrm{Bi}_{2} \mathrm{O}_{3}-20 \mathrm{~m} \% \mathrm{Er}_{2} \mathrm{O}_{3} \\
\text { with Pt deposition }\end{array}$ & $\begin{array}{l}3.0 \times 10^{-7} \\
6.6 \times 10^{-7}(\mathrm{f}) \\
\end{array}$ & $\begin{array}{l}600 \\
600 \\
\end{array}$ & $\begin{array}{l}\mathbf{a} \\
\mathbf{a}\end{array}$ & {$[230]$} \\
\hline $\mathrm{SrCo}_{0.8} \mathrm{Fe}_{0.2} \mathrm{O}_{3-\delta}$ & $\begin{array}{l}1.2 \times 10^{-3}(\mathrm{~h}) \\
1.2 \times 10^{-3}(\mathrm{~h}) \\
\end{array}$ & $\begin{array}{l}700 \\
450 \\
\end{array}$ & $\begin{array}{l}\mathbf{a} \\
\mathbf{a} \\
\end{array}$ & [242] \\
\hline $\mathrm{CeO}_{2}-30 \mathrm{~m} \% \mathrm{TbO}_{1.75}$ & $\begin{array}{l}1.0 \times 10^{-4} \\
5.6 \times 10^{-4} \\
\end{array}$ & $\begin{array}{l}600 \\
800 \\
\end{array}$ & $\begin{array}{l}\mathbf{a} \\
\mathbf{a}\end{array}$ & {$[242]$} \\
\hline
\end{tabular}

Data from ${ }^{18} \mathrm{O} /{ }^{16} \mathrm{O}$ exchange followed by (a) gas analysis or (b) dynamic SIMS analysis, and (c) from electrochemical exchange current density measurements; (d) single crystal, (e) added for comparison, (f) extrapolated value, (g) value at oxygen pressure $1.3 \times 10^{3}$ $\mathrm{Pa}$, (h) may be a limiting value due to a stagnant gas layer at the sample surface.

Values normalized to air at indicated temperature 
Wen and Mason [234] have shown that in oxygen pump experiments using scandia stabilized zirconia as the electrolyte with porous gold electrodes the cathodic overpotential is substantially decreased by adding a small amount (approximately 3 vol-\%) of water vapour to the oxygen feed stream. Consequently, higher pumping rates can be obtained at a given overpotential. Similar behaviour has been reported in other studies [235, 236].

As is discussed in somewhat more detail later (see Section 12.10), there is extensive experimental evidence for the promoting role of electronic conductivity in the surface exchange kinetics of oxide electrolytes. The electronic conductivity can be increased by doping the topmost layer by metal ions which can change their valence, e.g. from $\mathrm{Fe}^{2+}$ to $\mathrm{Fe}^{3+}$. Schouler et al. [237] showed the improvement of the water vapor reduction rate at the YSZ interface upon doping with cerium by a solid state diffusional process. Steele et al. [228] using the ${ }^{18} \mathrm{O}$-method observed a five-fold increase in the surface exchange rate for bismuth-implanted YSZ samples with respect to the unimplanted material. Van Hassel [238] showed the existence of a remarkably decreased electrode polarization, corresponding to an increase in the exchange current density, $i_{o}$, by ion implantation of YSZ with iron.

As has been mentioned already in Section 6 , it has been recognized in the literature that the use of mixed conducting oxide electrodes has intrinsic advantages over metallic electrodes in reducing electrode polarization and efficiency losses at oxide-gas interfaces in solid oxide fuel cells. The area of charge transfer would no longer be geometrically restricted to regions near the three phase boundary (gas, electrode, electrolyte) but occurs over the entire electrode-gas interfacial area (see Fig. 16).

Attention is drawn in Table 9 to the large values of the surface exchange rate of cobalt based perovskite-type oxide electrodes (as layers on calcia-doped ceria) as measured by Inou et al. [239, 240]. The $\mathrm{CeO}_{2}$ based sensor operating with an electrode of composition $\mathrm{La}_{0.6} \mathrm{Sr}_{0.4} \mathrm{Co}_{0.98} \mathrm{Ni}_{0.02} \mathrm{O}_{3}$ exhibited theoretical EMFs even at $200^{\circ} \mathrm{C}$. Similar results have been obtained by van Dijk et al. [241] when studying sputtered layers of fluorite-type $\mathrm{GdZr}_{2} \mathrm{O}_{7}$ on oxide electrolyte supports. As shown in Table 9, the results of Kurumchin and Perfil'ev show that the exchange rate increases dramatically if $\mathrm{PrO}_{2-\delta}$ is deposited on the surface of stabilized zirconia [230]. Preliminary results from the present writers' laboratory [242] for the exchange rates on several mixed conducting oxides are also given in this table.

Takeda et al. [243] studied cathodic polarization phenomena of perovskite oxide electrodes of the type $\mathrm{La}_{1-\mathrm{x}} \mathrm{Sr}_{\mathrm{x}} \mathrm{MO}_{3-\delta}$ with $\mathrm{M}=\mathrm{Cr}, \mathrm{Mn}, \mathrm{Fe}, \mathrm{Co}$, which were sputtered on yttria stabilized $\mathrm{ZrO}_{2}$. The cobalt compound had the highest electrode activity, the manganese and iron compounds had a much lower activity, and the chromium compound was least active. The authors propose that the charge transfer reaction on the cobalt compound is rate determining, both the oxide ion diffusivity in the solid and the oxygen dissociation on the surface being fast. On the iron and manganese 
compounds the oxygen dissociation is much slower and becomes rate determining, while in the chromium compound the oxide ion diffusion becomes so slow that it becomes the rate determining step.

\subsubsection{Relation with oxidation catalysis}

The ability of oxides for isotopic exchange has been recognized as a measure of oxide bond strength [244]. The observed activation enthalpies for isotopic exchange are close to the energies of oxygen bonding to the catalyst surface, taking into account details of the mechanism [204, 244]. The role of oxide bond strength, which is known to be decisive in a number of oxidation reactions, and which has also been mentioned in Section 4.2, is reflected in several close regularities seen in the rates of ${ }^{18} \mathrm{O}$ exchange on oxides and their corresponding behaviour as oxidation catalysts for various reactants, e.g. of 3d transition metals $\left(\mathrm{TiO}_{2}, \mathrm{~V}_{2} \mathrm{O}_{5}, \mathrm{Cr}_{2} \mathrm{O}_{3}, \mathrm{MnO}_{2}, \mathrm{Fe}_{3} \mathrm{O}_{4}, \mathrm{Co}_{3} \mathrm{O}_{4}, \mathrm{NiO}, \mathrm{CuO}, \mathrm{ZnO}\right.$ ) in the oxidation of hydrogen, methane, propene and nitrogen oxide [200, 201, 204]. Similar correlations have been reported for the rare earth oxide catalysts reviewed by Rosynek [213], $\mathrm{MFe}_{2} \mathrm{O}_{4}$ ferrites (where $\mathrm{M}=\mathrm{Mg}, \mathrm{Mn}, \mathrm{Co}, \mathrm{Ni}, \mathrm{Cu}, \mathrm{Zn}, \mathrm{Cd}$ ) [245, 246, 247], and for other compounds [199, 204].

It emerges from many catalytic oxidation studies, and it has been discussed by, e.g. Haber [248], that weakly bound chemisorbed oxygen species, which are thought of as 'electrophilic' are believed to be responsible for unselective oxidation. Lattice oxygen, thought of as 'nucleophilic' would promote selective oxidation.

The above idea can be generalized by adopting the simple two-step scheme discussed in the previous subsection. The two elementary steps in this model are: (1) primary activation of gas phase oxygen leading to oxygen in a chemisorbed state $\left(\mathrm{O}_{\text {ads }}^{-}\right.$, $\mathrm{O}_{2}^{-}$ads etc.) with rate $\mathrm{r}_{\mathrm{a}}$ and (2) its subsequent incorporation (and diffusion) in the oxide lattice with rate $r_{s}$. Accordingly, under conditions of equilibrium the concentrations of chemisorbed species are balanced by the respective rates $r_{a}$ and $r_{s}$. Data on isotopic exchange of, e.g., $\mathrm{Co}_{3} \mathrm{O}_{4}$ indicate that the rate of oxygen diffusion is far less than the rate of primary adsorption, i.e. $\mathrm{r}_{\mathrm{a}}$ " $\mathrm{r}_{\mathrm{s}}$. Thus one expects $\mathrm{Co}_{3} \mathrm{O}_{4}$ to be unselective in catalytic oxidation, as has indeed been observed. On the other hand the surface of, e.g., $\mathrm{V}_{2} \mathrm{O}_{5}$ remains homogeneous towards isotopic exchange due to a low binding rate of gas phase oxygen in combination with a high lattice mobility, i.e, $r_{a}$ " $r_{s}$, which is in agreement with its high selectivity as an oxidation catalyst. 


\section{OXYGEN PERMEATION}

A promising use of mixed conducting oxides is as semipermeable membranes, in particular when combined with catalytic activity, supplying or extracting oxygen in a partial pressure gradient without the need for external voltages. As described in Section 9.3 the transport of oxygen, arising from thermal dissociation of molecular species chemisorbed on the oxide surface, is made possible by the joint diffusion of oxygen anions, or vacancies, and electronic species through the membrane under the driving force of the gradient in chemical potential of $\mathrm{O}_{2}$.

Wagner's theory is usually adopted to express the oxygen flux across the membrane in terms of measurable electrical parameters [34]

$j_{O_{2}}=\frac{1}{4^{2} F^{2} L} \int_{\mu_{O_{2}}}^{\mu_{O_{2}}^{\mathrm{I}}} t_{\text {ion }} t_{\text {el }} \sigma_{\text {total }} d \mu_{O_{2}}$

In this equation $t_{\text {ion }}$ and $t_{\mathrm{el}}$ are the fractions of the total conductivity, $\sigma_{\text {total }}\left(=\sigma_{\text {ion }}+\right.$ $\sigma_{\mathrm{el}}$ ), which are provided by ionic and electronic defects (see Section 2.1 ), $\mathrm{L}$ is the thickness of the membrane and $F$ the Faraday constant. The limits of integration are the chemical potentials of molecular oxygen, $\mu_{\mathrm{O} 2}$, at the gas phase boundaries.

Wagner's theory is valid if one assumes that bulk oxide properties dominate the transport behaviour. However, Dou et al. [160] observed that the rate of oxygen permeation of calcia-stabilized zirconia (CSZ) is limited partly by diffusion within the solid and partly by the surface exchange reactions. The isothermal permeation of oxygen through CSZ tubes was studied at $960-1450^{\circ} \mathrm{C}$, one side of the specimen being exposed to oxygen pressures from $10^{-3}$ to $1 \mathrm{~atm}$, the other to pressures from $10^{-6}$ to $10^{-4} \mathrm{~atm}$. The characteristic thickness at which diffusion and surface processes are about equally determined by the extent of oxygen permeation was found to be $2.7 \times 10^{-2} \mathrm{~cm}$ at $1230^{\circ} \mathrm{C}$ and $\mathrm{p}_{\mathrm{O} 2}=1 \mathrm{~atm}$ (high pressure side). In later studies the same authors used the time-lag method to obtain the chemical diffusion coefficient of oxygen in CSZ [171, 249]. The results were consistent with a trapping mechanism of electron holes or with partial control by surface processes.

For the macroscopic description the membrane may tentatively be divided into three regions: a central bulk (Wagner) zone and adjacent interfacial zones, emphasizing the importance of both solid state diffusion and surface exchange reactions to the extent of oxygen permeation. The basic scheme is shown in Fig. 25. The available driving force for permeation, being the gradient in oxygen chemical potential, $\mu_{\mathrm{O} 2}$, is proportioned between the various zones. If the membrane is made sufficiently thick diffusion will be rate determining. A mixed type of rate control is encountered upon decreasing membrane thickness. Ultimately the surface reactions become rate determining for very thin membranes.

A competition between surface exchange reactions and bulk diffusion processes 


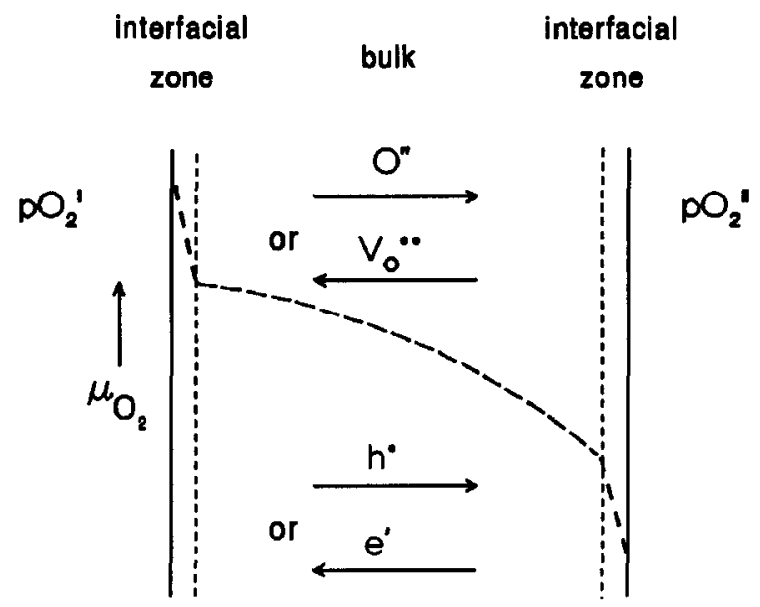

Fig. 25. Schematic representation of the gradient in oxygen chemical potential, $\mu_{\mathrm{O} 2}$, at various zones during steady state oxygen permeation.

has also been assumed by Bouwmeester et al. [224] to explain the isothermal semipermeability at $610-810{ }^{\circ} \mathrm{C}$ of dense sintered disks of bismuth oxide stabilized with 25 mol-\% erbia (BE25). The kinetic order with respect to $\mathrm{O}_{2}$ and the activation enthalpy were found to increase gradually with decreasing specimen thickness (or temperature) due to the changeover from diffusion controlled to surface controlled kinetics. The results enable estimation of both the (p-type) electronic conductivity of BE25 and the surface exchange rate. The value obtained for the latter showed excellent agreement with that from ${ }^{18} \mathrm{O}-{ }^{16} \mathrm{O}$ isotopic exchange.

Teraoka et al. [61, 250, 251] have studied oxygen permeation for a large number of $\mathrm{LnCoO}_{3}$ based perovskite type oxide systems. The partial substitution of $\mathrm{A}$ and B site cations drastically affects the oxygen semipermeability, mainly due to the concomitant change in the concentration of oxygen vacancies. For compounds $\mathrm{La}_{1-\mathrm{x}} \mathrm{Sr}_{\mathrm{x}} \mathrm{Co}_{1-\mathrm{y}} \mathrm{Fe}_{\mathrm{y}} \mathrm{O}_{3-\delta}$ the ionic conductivity, calculated from the permeation fluxes, was found to be in good agreement with that measured directly [61]. This is to be expected because the electronic conductivity in these compounds is so high that the permeation flux has to be determined by the ionic conductivity. The highest permeation rate, viz. $0.023 \mathrm{~mol} \cdot \mathrm{m}^{-2} . \mathrm{s}^{-1}$ at $800^{\circ} \mathrm{C}$ and oxygen pressure gradient $1 \mathrm{~atm} v \mathrm{~s}$. helium gas, as found through $1 \mathrm{~mm}$ thick ceramics of $\mathrm{SrCo}_{0.8} \mathrm{Fe}_{0.2} \mathrm{O}_{3}$ [250], is only slightly smaller than those generally found for meso-porous membranes $\left(0.1-0.3\right.$ mol.m $\mathrm{m}^{-2} . \mathrm{s}^{-1}$ for $\mathrm{O}_{2}$ (and $\left.\mathrm{H}_{2}\right)$ for a membrane with thickness $5 \mu \mathrm{m}$ with pores $3 \mathrm{~nm}$ in diameter and pressure gradient $1 \mathrm{~atm} v s$. vacuum) and is even larger than that observed for microporous membranes [252] $\left(0.01-0.001 \mathrm{~mol} . \mathrm{m}^{-2} . \mathrm{s}^{-1}\right.$ for a membrane layer of $50 \mathrm{~nm}$ with $0.5-1.0 \mathrm{~nm}$ pores and 
pressure gradient 1 atm vs. vacuum). Preliminary results of ${ }^{18} \mathrm{O}-{ }^{16} \mathrm{O}$ isotopic exchange confirmed the expected high exchange rates for the compound $\mathrm{SrCo}_{0.8} \mathrm{Fe}_{0.2} \mathrm{O}_{3}[242$ ] (see Table 9, Section 9.4.4).

The existence of a semipermeability flux due to a nonvanishing electronic conductivity in solid electrolytes such as CSZ and BE25 can be detrimental in high-temperature oxygen gauges. This has been discussed by Maskell and Steele [253] and by Kleitz et al. [254, 255].

On the other hand the permeating flux can be used advantageously if it is associated with specific surface reactions. Nigara and Cales [256] investigated the production of $\mathrm{CO}$ by direct thermal decomposition of $\mathrm{CO}_{2}$ (see also Section 12.8). In a previous study Cales and Baumard [257] reported the production of $\mathrm{H}_{2}$ from $\mathrm{H}_{2} \mathrm{O}$ utilizing this method and attributed the low efficiency of this process to the low rate of dissociation of $\mathrm{H}_{2} \mathrm{O}$.

Di Cosimo et al. [258] studied the oxidative dehydrodimerization of propene at $600{ }^{\circ} \mathrm{C}$ using $\left(\mathrm{Bi}_{2} \mathrm{O}_{3}\right)_{0.85}\left(\mathrm{La}_{2} \mathrm{O}_{3}\right)_{0.15}$ as catalyst membrane separating a feed of $20 \%$ propene in helium on one side from air on the other side. This reaction is discussed in more detail in Section 12.5. 


\section{CATALYTIC REACTIONS}

\subsection{Types of reactions}

Catalytic reactions can be divided into different groups depending on whether only the total activity (or conversion) is of importance, or whether the selectivity is also an important variable. Some examples of the former group are:

$2 \mathrm{CO}+\mathrm{O}_{2} \rightarrow 2 \mathrm{CO}_{2}$

$2 \mathrm{SO}_{2}+\mathrm{O}_{2} \rightarrow 2 \mathrm{SO}_{3}$

Some reactions in which the selectivity is also important are:

$6 \mathrm{NO}+4 \mathrm{NH}_{3} \rightarrow 2 \mathrm{~N}_{2}+6 \mathrm{H}_{2} \mathrm{O}$

$2 \mathrm{CH}_{3} \mathrm{OH}+\mathrm{O}_{2} \rightarrow 2 \mathrm{CH}_{2} \mathrm{O}+2 \mathrm{H}_{2} \mathrm{O}$

$\mathrm{CH}_{2}=\mathrm{CH}-\mathrm{CH}_{3}+\mathrm{O}_{2} \rightarrow \mathrm{CH}_{2}=\mathrm{CH}-\mathrm{CHO}+\mathrm{H}_{2} \mathrm{O}$

$\mathrm{CO}$ oxidation is a relatively simple reaction which has been chosen by many authors as model reaction for testing different types of catalyst systems. One special advantage of this reaction that there are no selectivity problems: there is only one product. Moreover the mechanism is rather well understood [259] so that an interpretation of the role of the catalyst is possible. The mechanism proposed consists of the following steps:

$$
\begin{aligned}
& \mathrm{CO}_{\mathrm{g}}+\mathrm{h}^{\bullet}=\mathrm{CO}_{\mathrm{ads}}^{+} \\
& \mathrm{CO}_{\mathrm{ads}}^{+}+\mathrm{O}_{\mathrm{O}}^{\mathrm{x}}=\mathrm{CO}_{2, \mathrm{ads}}^{-}+\mathrm{V}_{\mathrm{O}}^{\infty} \\
& \mathrm{CO}_{2, \mathrm{ads}}^{-}+\mathrm{h}^{\bullet}=\mathrm{CO}_{2, \mathrm{~g}} \\
& \mathrm{CO}_{2, \mathrm{ads}}^{-}+\mathrm{O}_{\mathrm{O}}^{\mathrm{x}}+\mathrm{h}^{\cdot}=\mathrm{CO}_{3, \mathrm{ads}}^{2-}+\mathrm{V}_{\mathrm{O}}^{\infty} \\
& \mathrm{CO}_{3, \mathrm{ads}}^{2-}+\mathrm{V}_{\mathrm{O}}^{\infty}=\mathrm{CO}_{2, \mathrm{~g}} \\
& \mathrm{O}_{2, \mathrm{~g}}=\mathrm{O}_{2, \mathrm{ads}}^{-}+\mathrm{h}^{\cdot} \\
& \mathrm{O}_{2, \mathrm{ads}}^{-}=\mathrm{O}_{2, \mathrm{ads}}^{2-}+\mathrm{h}^{\bullet} \\
& \mathrm{O}_{2, \mathrm{ads}}^{2-}=2 \mathrm{O}_{\mathrm{ads}}^{-} \\
& \mathrm{O}_{\mathrm{ads}}^{-}+\mathrm{V}_{\mathrm{O}^{\infty}}^{\infty}=\mathrm{O}_{\mathrm{O}}^{\mathrm{x}}+\mathrm{h}^{\bullet}
\end{aligned}
$$


In this mechanism the first four steps represent the reduction of the catalyst, accompanied by oxidation of $\mathrm{CO}$, the last four steps represent the reoxidation of the catalyst by gaseous oxygen. In many cases it can be assumed that the equilibrium between electrons and electron holes (Eqn. (4)) is established. In those cases Eqns. (46) - (54) can be written with $\mathrm{e}^{\prime}$ instead of $\mathrm{h}^{\circ}$, but in general that type of electronic defect is chosen which is supposed to be the majority electronic charge carrier in the oxide. On the other hand, in many cases it is not known which of the electronic charge carriers participates in the reactions, and this may well be the minority electronic charge carrier in certain cases. The oxidation of carbon monoxide is discussed more extensively in Section 12.2.

An important catalytic reaction is NO reduction which is applied in the cleaning of flue and exhaust gases. Rosynek [213] discusses oxygen exchange as well as the conversion of nitrogen oxides and in particular the investigations of this reaction by Winter [260, 261, 262]. The activation enthalpies and rate of decomposition of $\mathrm{N}_{2} \mathrm{O}$ on the different oxides are nearly identical to those for the exchange of lattice oxygen, indicating a common rate determining step of these reactions, which is probably the desorption of $\mathrm{O}_{2}$. The catalytic reduction of nitrogen oxides is treated in more detail in Section 12.3.

Promising catalysts based on bismuth-rich, layered solid oxychlorides for the oxidation of methane to higher hydrocarbons have been found by Ueda and Thomas [263]. An interesting observation made by these authors is that poorly crystallized materials show poor catalytic properties. On the one hand this may suggest that mobility of lattice oxygen is of importance in these reactions. On the other hand, however, it may also be that the disturbed surface leads to less favourable conditions for adsorption and surface reaction. The mobility of defects is expected to be lower and not higher in a disturbed or poorly crystallized lattice when compared with that in a normal lattice. This means that according to the present writers the second explanation is probably correct.

Selective oxidation of unsaturated hydrocarbons has been widely studied [111] too and is discussed in some detail in Section 12.4. Further important catalytic reactions, such as hydrocarbon combustion, dehydrogenation and dehydrodimerization, etc. are also considered in Section 12.

\subsection{Catalyst materials}

Of course, many different materials which have been studied as catalysts are discussed or mentioned in this review. In this section some remarks are made about materials which seem to show promise as catalysts or which have been studied but are not explicitly considered elsewhere in this review.

The electronic structure of $\mathrm{MoO}_{3}$ and of several modifications of $\mathrm{Bi}_{2} \mathrm{O}_{3}$ was studied by Anderson et al. [264], using a semi-empirical molecular orbital analysis of atom superposition and electron delocalization. The results enabled the authors to explain the relative lability for oxygen exchange of $\mathrm{Bi}_{2} \mathrm{O}_{3}$ and also the much smaller 
tendency for oxygen exchange of $\mathrm{MoO}_{3}$. This type of study, which has not been applied very often, gives valuable information in addition to that obtained from a study of the bulk properties of the catalyst materials.

A large number of catalytic reactions have been studied using a great variety of different perovskite type materials as catalysts. A survey of these studies has been given by Yamazoe and Teraoka [265].

Bonanos et al. [266] have studied the ionic and mixed conducting properties of gadolinium-doped barium cerate perovskites of composition $\mathrm{BaCe}_{1-\mathrm{x}} \mathrm{Gd}_{\mathrm{x}} \mathrm{O}_{3-\mathrm{x} / 2}$. Below $500{ }^{\circ} \mathrm{C}$ these are mainly ion conductors. At high temperatures and high partial pressures of oxygen they become mixed conductors. At $600{ }^{\circ} \mathrm{C}$ these compounds exhibited the highest known component of oxygen ion conductivity. Because of the special role of both $\mathrm{Bi}$ and of $\mathrm{Ce}$ in oxidation catalysis, a closer examination of the catalytic properties of these compounds certainly seems worthwhile.

Abraham et al. [267] recently introduced a new class of oxygen ion conductors derived from $\mathrm{Bi}_{4} \mathrm{~V}_{2} \mathrm{O}_{11}$ by partial substitution of vanadium by other metallic ions. Again, in relation to the interesting catalytic properties of $\mathrm{Bi}$ and $\mathrm{V}$, these compounds also merit further study of their catalytic behaviour.

Two reviews by Voorhoeve et al. $[11,268]$ concerning oxides with the perovskite structure, in which these are discussed from the standpoint of the application of materials science in catalysis, and a similar review by Sleight [12] concerning scheelite type oxides have already been mentioned earlier.

Most of the investigations covered by this review are devoted to the study of catalyst materials in bulk form because the solid state properties are then expected to show their influence best. On the other hand many catalysts in practice are supported on other materials, again mostly oxides. A review especially devoted to the properties of supported perovskites has been published by Mizuno [269]. One special problem is the interaction between the catalyst and the support. For example cobaltates cannot be supported on $\mathrm{Al}_{2} \mathrm{O}_{3}$, a commonly used support material, because the cobalt is extracted from the perovskite to form the very stable aluminium-cobalt spinel. An interesting effect is that, e.g., $\mathrm{LaCoO}_{3}$ disperses so well over $\mathrm{ZrO}_{2}$ that this becomes by far the most active form (expressed per gram of catalyst) due to its very large specific surface area. On the other hand the strontium doped lanthanum cobaltate, which is expected to be even more active, cannot be used on $\mathrm{ZrO}_{2}$ because the strontium is taken up by the support to form the very stable strontium zirconate.

The importance of the type and surface structure of catalysts for the selectivity of oxidation reactions has been suggested and discussed by Callahan and Grasselli [270]. These authors propose that for a catalyst to be selective in hydrocarbon oxidation two conditions must be met. In the first place the oxygen atoms must be distributed on the surface of the catalyst in such a way that the number of active oxygen atoms in isolated groups is limited. In the second place the binding energy of the active oxygen atoms must 
be such that both rapid removal in the oxidation of the hydrocarbon and rapid uptake in regeneration of the catalyst is assured. As the authors point out, the first requirement can also be met when there are oxygen atoms with different binding energies on the catalyst surface. Thus those materials in which there are at least two types of oxygen (see for example the corresponding oxygen potentials in Table 3 ) can show selective oxidation if the second requirement is also met. It should be noted that when there is sufficient mobility of oxygen through the lattice it is of course possible that oxidation of the hydrocarbon and reoxidation of the catalyst take place at different sites.

\subsection{Kinetics of catalytic reactions}

Catalytic oxidation is often described by power law kinetic equations. Thus for a reaction:

$$
\mathrm{aS}+\mathrm{bO}_{2}=\mathrm{P}+\mathrm{Q}
$$

where $\mathrm{S}$ is the substrate which is oxidized and $\mathrm{P}$ and $\mathrm{Q}$ are oxidation products, the kinetic equation is written as:

$\mathrm{r}=\mathrm{k} \times \mathrm{p}_{\mathrm{S}}^{\mathrm{m}} \times \mathrm{p}_{\mathrm{O}_{2}}^{\mathrm{n}} \times \mathrm{p}_{\mathrm{P}}^{\mathrm{v}}$

In this equation $r$ is the reaction rate; $k$ the rate constant; $p_{i}$ the partial pressure of $i$, and $\mathrm{m}, \mathrm{n}, \mathrm{v}$ are the reaction orders. If any of these are negative the corresponding compound is called an inhibitor for the reaction. More meaningful equations are those derived from the oxidation-reduction model of Mars and van Krevelen [271]:

$$
\mathrm{r}=\frac{1}{\frac{1}{\mathrm{k}_{\mathrm{ox}}\left[\mathrm{O}_{2}\right]}+\frac{1}{\mathrm{k}_{\mathrm{red}}[\mathrm{S}]}}
$$

and from the Langmuir - Hinshelwood mechanism (see, e.g., [272]):

$$
\mathrm{r}=\frac{\mathrm{k}_{\mathrm{r}}[\mathrm{S}]\left[\mathrm{O}_{2}\right]^{\mathrm{n}}}{1+\mathrm{K}_{\mathrm{O}_{2}}\left[\mathrm{O}_{2}\right]+\mathrm{K}_{\mathrm{S}}[\mathrm{S}]+\mathrm{K}_{\mathrm{P}}[\mathrm{P}]}
$$

with $\left[\mathrm{O}_{2}\right]$ is the oxygen concentration, $[\mathrm{S}]$ the substrate concentration, $[\mathrm{P}]$ the product concentration, $k_{i}$ are reaction rate constants and $K_{i}$ are adsorption constants. In the derivation of Eqn. (58) it is assumed that oxygen, the substrate and a product are all adsorbed on the same surface sites.

However, the power law is often used as an approximation to the equations derived from the Mars - van Krevelen or Langmuir - Hinshelwood mechanisms. In terms of the oxidation-reduction model a value of $m$ close to 1 and $n$ close to zero in the power law equation corresponds with the reduction of the catalyst being rate determining, i.e. the term with $k_{o x}$ in Eqn. (57) being negligible, whereas a value of $m$ close to zero and 
n close to one indicates that reoxidation of the catalyst is rate determining, i.e. the term with $\mathbf{k}_{\text {red }}$ in Eqn. (57) is negligible. Similar relations can be given between the exponents in the power law kinetic equation and limiting cases for different types of Langmuir Hinshelwood mechanism.

A square root dependence of the reaction rate on the oxygen partial pressure is often observed. This can be rationalized by assuming that, in the rate determining step, the molecule to be oxidized reacts with dissociatively adsorbed oxygen:

$\mathrm{O}_{2}=2 \mathrm{O}_{\text {ads }}$

$\mathrm{S}+\mathrm{O}_{\mathrm{ads}} \rightarrow$ Products

which leads to a rate equation of the form:

rate $=k \times p_{S} \times p_{O_{2}}^{1 / 2}$

In the derivation of this equation the possible influence of, e.g., charge transfer on the rate equation is left out of consideration. This type of rate equation can be observed both for a Langmuir - Hinshelwood mechanism, i.e. with adsorbed S, and for an Eley -Rideal mechanism, i.e. with gas phase $S$ reacting with adsorbed oxygen atoms. 


\section{MECHANISM OF CATALYTIC REACTIONS ON SOLID ELECTROLYTES}

\subsection{Introduction}

In any catalytic reaction there are a number of successive steps, as pointed out for the oxygen exchange reaction (see Fig. 21). These are shown schematically in Fig. 26 and those which are of main interest here are the adsorption, surface reaction and desorption steps.

\section{Steps in Catalytic Reaction \\ 1 transport of reactants from gas phase to surface \\ - external diffision \\ - internal diffusion through pores \\ 2 adsorption on surface \\ - including surface diffusion \\ 3 reaction on surface \\ 4 desorption from surface \\ - including surface diffusion \\ 5 transport of reactants from \\ surface to bulk of gas \\ - internal diffusion through pores \\ - externar diffusion}

Fig. 26. Steps in catalytic reaction.

There are many different ways to describe the mechanism of catalytic oxidation reactions [272]. Voorhoeve [11, 273] has introduced the terms suprafacial and intrafacial mechanisms. In a suprafacial mechanism the reaction between adsorbed species on the surface and the adsorption-desorption reactions are much faster than the reactions in which oxygen from the oxide lattice is involved. An intrafacial mechanism in contrast is one in which the removal of oxygen from the catalyst or the reverse are of comparable or greater rate than the catalytic surface reaction. In the latter type of mechanism the valence state of the catalyst rapidly adjusts itself to the external conditions. The special properties of ion or mixed conductors are expected to be of particular importance in catalytic reactions which show an intrafacial mechanism.

\subsection{Carbon monoxide oxidation}

As remarked above the oxidation of carbon monoxide has been widely studied as a model reaction. Rosynek [213] reviews older work on the carbon monoxide oxidation catalysed by the rare earth oxides. Just as in the oxygen exchange reaction considered in Section 9, the rate of the carbon monoxide oxidation increases with increasing mobility of lattice oxygen ions. In this respect the work of Claudel et al. [274, 275] on carbon monoxide oxidation over $\mathrm{CeO}_{2}$ must also be mentioned.

Berkstresser et al. [276] studied the oxidation of carbon monoxide on $\mathrm{ZrO}_{2}$, $\mathrm{Zr}_{0.91} \mathrm{Ca}_{0.09} \mathrm{O}_{1.91}$ and on $\mathrm{Th}_{0.85} \mathrm{La}_{0.15} \mathrm{O}_{1.925}$. The first is an intrinsic conductor with low conductivity, the second is a nearly pure ion conductor and the third a mixed conductor with an ionic transference number of about 0.9 . The catalytic activity, determined as the temperature at which a certain conversion is reached, clearly increased in this order. Because this is measured using the same weight of catalyst, while the surface area of the 
thorium containing catalyst is much smaller than that of the others, the difference is even larger than suggested. The lack of exact surface area data makes it difficult to compare the results with those of some other catalysts $\left(\mathrm{LaCoO}_{3}, \mathrm{NiO}\right)$ used in the same investigation. The results support the idea that for this reaction the rate determining step involves the combined movement of oxygen ions and of electrons (or electron holes) as indicated in the mechanism of Eqns. (46) - (54). In fact the authors propose that the catalytic activity can be expected to increase with increasing $t_{\text {ion }} \times t_{\text {el }}$, which is in agreement with the experimental results and with the Wagner model for transport in oxides.

A particularly elegant demonstration of the involvement of lattice oxygen in the oxidation of carbon monoxide, using nonstoichiometric praseodymium oxide and terbium oxide as catalysts, has been given by Takasu et al. $[146,147]$. When $\mathrm{C}^{16} \mathrm{O}$ is oxidized over praseodymium oxide or terbium oxide with ${ }^{18} \mathrm{O}_{2}$ present in the gas phase the main product is $\mathrm{C}^{16} \mathrm{O}_{2}$. Thus the oxygen in the product initially comes only from the oxide. The reoxidation of the oxide, of course, occurs by ${ }^{18} \mathrm{O}_{2}$ but either this oxidation takes place at other surface sites than are used for $\mathrm{CO}$ oxidation, or else rapid isotopic mixing occurs in the oxide due to a high oxygen diffusivity. As a consequence it takes a rather long time before significant amounts of $\mathrm{C}^{16} \mathrm{O}^{18} \mathrm{O}$ are formed. This is supported by the observation that the $\mathrm{CO}_{2}$ formed after $2 \mathrm{~h}$ of reaction was equivalent to only $3.3 \%$ of the total lattice oxygen present in the catalyst.

Van Dijk et al. [277, 278] and ter Maat et al. [259] studied the catalytic properties of oxides with the fluorite or the related pyrochlore structure. In lanthanide titanates $\mathrm{Ln}_{2} \mathrm{Ti}_{2} \mathrm{O}_{7}$ the order in $\mathrm{O}_{2}$ of $\mathrm{CO}$ oxidation increases with decreasing radius of the Ln ion, meaning an increased bond strength with oxygen ions, indicating that lattice oxygen ions are the active species in this reaction. From this it can be concluded that this reaction shows an intrafacial mechanism. Substitution of bismuth, giving $\mathrm{Ln}_{1-\mathbf{x}} \mathrm{Bi}_{\mathbf{x}} \mathrm{Ti}_{2} \mathrm{O}_{7}$, leads to a strong decrease of the order in $\mathrm{O}_{2}$, indicating that reoxidation is fast, in agreement with the literature [228, 279], where it is suggested that bismuth accelerates adsorption and/or surface diffusion of oxygen. Although the experimental evidence for this high activity is clear, there is as yet no real explanation why bismuth shows this behaviour.

For zirconates with the general formula $\mathrm{Nd}_{2}\left(\mathrm{Zr}_{1-\mathrm{x}} \mathrm{Ce}_{\mathrm{x}}\right)_{2} \mathrm{O}_{7}$ a clear influence of ordering due to heat treatment was observed. In low ordered compounds the reduction of the catalyst is mainly rate determining (low order in oxygen, high in carbon monoxide). For the more highly ordered compounds the reverse is found, the reoxidation there being mainly rate determining. In both groups increasing the cerium concentration leads to increased activity. It was not possible to conclude unambiguously whether the change in behaviour above a certain minimum cerium content was due to a change in the type of electronic conductivity (n-type or p-type) in these mixed conductors or merely to the introduction of a new type of redox couple. 
The presence of terbium in terbium-gadolinium zirconates $\left(\left(\mathrm{Tb}_{\mathrm{x}} \mathrm{Gd}_{1-\mathrm{x}}\right)_{2} \mathrm{Zr}_{2} \mathrm{O}_{7}\right)$, which leads to mixed conductivity, affects the kinetic behaviour and the catalytic activity of the materials investigated, which have the fluorite or pyrochlore structure [280]. This is a consequence of the increase in the electronic charge carrier concentration due to the valence change of terbium:

$\mathrm{Tb}^{4+}+\mathrm{e}^{-}=\mathrm{Tb}^{3+}$

Increasing the bismuth content in $\left(\mathrm{Tb}_{\mathrm{x}} \mathrm{Bi}_{1-\mathrm{x}}\right)_{2} \mathrm{Zr}_{2} \mathrm{O}_{7}$ leads to an increased oxidation activity. Because this is accompanied by an increase in the order in carbon monoxide and a decrease in that of oxygen this supports again the suggestion that bismuth facilitates the uptake of oxygen.

In this case, too, a large influence of the heat treatment of the catalysts was observed, indicating the possible influence of the ordering, although a change in surface composition might also explain the experimental results. Even when the ordering is still very small and only present in the form of microdomains an increased activity is already observed. The activity first increases further with increasing ordering but then decreases again at still higher ordering. A possible explanation is that, in particular, the boundary between the ordered and disordered parts comprises the most active sites on the surface. This is similar to the proposal by Andersson [281] in explaining similar effects observed in the oxidation of aromatic hydrocarbons. A more extensive discussion of interfacial effects on catalytic reactions on different oxides has been published by Courtine [282].

Otsuka and Kunitomi [283] studied the oxidation of carbon monoxide over praseodymium oxides both in the presence and in the absence of gaseous oxygen. The kinetics were practically identical and the reaction is zero order in oxygen. Both observations suggest that the rate determining step is the reaction between carbon monoxide and the lattice oxygen atoms of $\mathrm{PrO}_{\mathrm{x}}$

The oxidation of carbon monoxide on a number of perovskite cobaltites $\mathrm{Ln}_{1-\mathrm{x}} \mathrm{M}_{\mathrm{x}} \mathrm{CoO}_{3}$ (with $\mathrm{Ln}=\mathrm{La}, \mathrm{Sm}$ and $\mathrm{M}=\mathrm{Pb}, \mathrm{Ba}, \mathrm{Th}$ ) was studied by $\mathrm{Rao}$ and Chakrabarty [284]. From the experimental result that substitution of the lanthanide both by divalent and by tetravalent ions increases the catalytic activity it was concluded that a direct correlation with the valency of the cobalt ion is not possible. Furthermore the catalytic activity was found to depend strongly on the preparation conditions of the catalysts, in particular on oxygen pretreatment. From conductivity measurements it was concluded that beyond $\mathrm{x}=0.2$ in the case of substitution by $\mathrm{Ba}$, oxygen vacancies are formed in an increasing amount with $x$. Because this is also the direction in which the catalytic activity increases this is clearly connected with the oxygen deficiency of these cobaltites.

Tascon et al. [285] performed a kinetic study of the oxidation of carbon monoxide on $\mathrm{LaCoO}_{3}$ at relatively low temperatures: 393 - $428 \mathrm{~K}$. The kinetic data agree satisfactorily with a mechanism in which the rate controlling step is the surface reaction 
between adsorbed carbon monoxide and dissociatively adsorbed oxygen, giving a carbonate species as intermediate. Both carbon monoxide and carbon dioxide adsorb on lattice oxygen ions. In agreement with others this means that at these low temperatures this oxidation shows a suprafacial mechanism.

Yamazoe and Teraoka have recently given a compilation of a large number of studies of $\mathrm{CO}$ oxidation using various perovskite type materials as catalysts [265].

Tabata et al. [286] studied the oxidation of carbon monoxide on perovskites of the types $\mathrm{La}_{1-\mathrm{x}} \mathrm{Th}_{\mathrm{x}} \mathrm{CoO}_{3}$ and $\mathrm{La}_{1-\mathrm{x}} \mathrm{Ce}_{\mathrm{x}} \mathrm{CoO}_{3}$ (with $\mathrm{x}$ ranging from 0 to 0.03 ) in relation to the surface chemical states studied with XPS. The catalytic activity showed a maximum at $x=0.02$. This correlated with a maximum in ionicity of the bonds between cobalt and lattice oxygen and a maximum in covalency of the bonds between cobalt and adsorbed oxygen. The ionicity of the cobalt-oxygen bonds, both for lattice oxygen and for adsorbed oxygen were determined from the binding energy difference $\mathrm{Co}_{2} \mathrm{p}_{3 / 2}-\mathrm{O} 1 \mathrm{~s}$ obtained from XPS spectra and from the interatomic Auger transitions. The authors neither give an explanation for these correlations nor do they indicate a reason for the sharp peak in catalytic activity and the ionicity of the cobalt-oxygen bonds at the rather low value of $\mathrm{x} \approx 0.02$.

The catalytic properties of perovskites of the type $\mathrm{La}_{1-x} \mathrm{Sr}_{x} \mathrm{CoO}_{3}$ for the oxidation of carbon monoxide were studied by Nakamura et al. [287, 288]. The reducibility of the catalysts and the ease of desorption of oxygen increased with $x$, while the rate of reoxidation became slower. The rate of transport of oxygen through the lattice and the ability to activate the oxygen molecule increased with $x$. These properties are reflected in the catalytic behaviour, which can be fitted to the oxidation-reduction mechanism discussed in Section 11.3. These results are also in agreement with the defect properties of this type of material discussed in Section 6 (see also Ref. 90).

The catalytic activity in carbon monoxide oxidation of supported perovskites of the type $\mathrm{La}_{1-\mathrm{x}} \mathrm{M}_{\mathrm{x}} \mathrm{MnO}_{3}$ with $\mathrm{M}=\mathrm{Sr}$ and $\mathrm{Pb}$ was investigated by Gallagher et al. [289]. The precipitation technique used made it possible to obtain surface areas of the perovskite of 1 to $44 \mathrm{~m}^{2} \mathrm{~g}^{-1}$. For constant composition, the activity was observed to be proportional to the surface area. On the other hand strontium substituted oxides were more active than lead substituted ones and they were also more stable with respect to reduction. At the beginning of the reduction process oxygen vacancies are formed, but at higher degrees of reduction metallic lead can be formed in the case of the lead containing materials.

\subsection{Reduction and decomposition of nitrogen oxides}

Nitrogen oxides (often designated as $\mathrm{NO}_{\mathrm{x}}$ ) are serious air pollutants and are formed in many high temperature combustion processes. A large research effort is therefore being devoted to methods for suppressing the release of these compounds into the atmosphere. Possible ways to reach this goal are the catalytic reduction of $\mathrm{NO}_{\mathrm{x}}$ by 
compounds such as carbon monoxide or ammonia or the catalytic decomposition into nitrogen and oxygen.

Voorhoeve [11] classified the reduction of NO, using perovskites as catalysts, as an intrafacial reaction, consisting of two steps: reduction of the catalyst under formation of oxygen vacancies and reoxidation by NO, for example:

$$
\begin{aligned}
& \mathrm{CO}+\mathrm{O}_{\mathrm{O}}+2 \mathrm{~h}^{\bullet} \rightarrow \mathrm{V}_{\mathrm{O}}^{\infty}+\mathrm{CO}_{2} \\
& \mathrm{v}_{\mathrm{O}}^{\infty}+\mathrm{NO} \rightarrow 1 / 2 \mathrm{~N}_{2}+\mathrm{O}_{\mathrm{O}}+2 \mathrm{~h}^{\bullet}
\end{aligned}
$$

Chu and Rohr [290] studied perovskite type solid solutions of the type $\mathrm{La}_{1-x} \mathrm{Sr}_{x} \mathrm{~B}_{1-\mathrm{y}} \mathrm{A}_{\mathrm{y}} \mathrm{O}_{3}$ (B = Mn, Fe, Co, Ni, or Cr; $\mathrm{A}=\mathrm{Fe}$ or $\mathrm{Cu}$ ) in the reduction of NO by $\mathrm{NH}_{3}$ and by carbon monoxide. In the reaction with carbon monoxide lattice oxygen vacancies, created by the reduction of the catalyst by carbon monoxide, play a large role in the reduction of NO, which is thus an intrafacial process. However, these compounds also show catalytic activity for the oxidation of carbon monoxide by $\mathrm{O}_{2}$, which is present in these gases too. There is no information from these measurements about the influence of the conducting properties of these compounds.

Chien et al. [291] investigated a number of different oxides, including the perovskites $\mathrm{La}_{0.85} \mathrm{M}_{0.15} \mathrm{CoO}_{3}$, with $\mathrm{M}=\mathrm{Ba}, \mathrm{Ca}$, $\mathrm{Hf}$ and $\mathrm{Sr}$, for the reaction between carbon monoxide and NO. In all cases the reaction was zero order in carbon monoxide and fractional order $(0.75-0.85)$ in NO. Unfortunately the comparison of the activity of the different catalysts is hampered by the fact that they are only expressed per gram of catalyst and the surface areas are not given. After pre-reduction of the oxide by carbon monoxide the rates are increased and this is correlated with an increased rate of adsorption of NO. The authors suggest that this was due to faster adsorption of NO on $\mathrm{Co}^{2+}$ compared with $\mathrm{Co}^{3+}$. In the light of other results presented in this review it could also be explained by an increase in the concentration of oxygen vacancies, which could also be the cause of the higher activity of, e.g., the barium doped material. On the other hand the hafnium doped perovskite, where the $V_{O}{ }^{*}$ concentration is expected to be decreased, has an activity of the same order as the other perovskites.

Mizuno et al. [292] studied the reaction between NO and carbon monoxide using the superconducting perovskite-type compound $\mathrm{YBa}_{2} \mathrm{Cu}_{3} \mathrm{O}_{\mathrm{y}}$ as catalyst. They observed an induction period during which a large amount of NO is absorbed, on a mole basis about 0.7 of the perovskite, followed by absorption of carbon monoxide. These then start to react under formation of $\mathrm{CO}_{2}$ and $\mathrm{N}_{2}$ until the stationary catalytic reaction between carbon monoxide and NO is finally established. Tabata et al. [293] and Arakawa et al. [294] also observed a very large absorption of NO which, when no other reactants are present, mainly desorbs unchanged at increased temperatures, only a small amount reacting to form $\mathrm{N}_{2}$.

Pomonis and Vickerman [295] have studied the catalytic decomposition of $\mathrm{N}_{2} \mathrm{O}$ on the mixed oxide catalysts $\mathrm{Sn}_{1-\mathrm{x}} \mathrm{V}_{\mathrm{x}} \mathrm{O}_{2}$ and $\mathrm{Ti}_{1-\mathrm{x}} \mathrm{V}_{\mathrm{x}} \mathrm{O}_{2}$ with $\mathrm{x}$ in the range of 0 to 0.2 . In 
the main the variations of the activity could be explained on the basis of the electronic state associated with a vanadium sublattice in the catalyst. The possible influence of the ionic and/or the electronic conductivity in these mixed oxides is not discussed.

The decomposition of $\mathrm{NO}$ on perovskites of the type $\mathrm{SrFeO}_{3-\mathrm{x}}$ was studied by Shin et al. [296] who observed both catalytic and non-catalytic decomposition of NO on this oxygen-deficient material. They proposed a redox mechanism in which oxygen vacancies play a central role as the sites at which the NO molecules are bound to the catalyst. Subsequently an electron is donated to $\mathrm{NO}$ by a $\mathrm{Fe}^{3+}$ ion and the resulting, unstable $\mathrm{NO}^{-}$ reacts with a second $\mathrm{NO}^{-}$giving $\mathrm{N}_{2}$. The oxygen atoms which are left behind then also combine and desorb as $\mathrm{O}_{2}$, reforming oxygen vacancies. Teraoka et al. [297] studied this reaction on a large number of perovskites and found the cobaltite $\mathrm{La}_{0.8} \mathrm{Sr}_{0.2} \mathrm{CoO}_{3}$ to be the most active at temperatures above $773 \mathrm{~K}$. Here also the presence of oxygen vacancies is essential, but the clear influence of the transition metal leads to the suggestion that the charge transfer reaction may also influence the rate of the catalytic reaction.

\subsection{Selective oxidation of olefins}

Many papers have appeared on the mechanism of selective oxidation reactions with bismuth molybdate catalysts, see e.g. Refs. 298, 299, 300, 301, 302, 303 and the reviews by Grasselli and Burrington [304], that by Grasselli [305], who also consider the related ammoxidation reaction, and by Snyder and Hill [306].

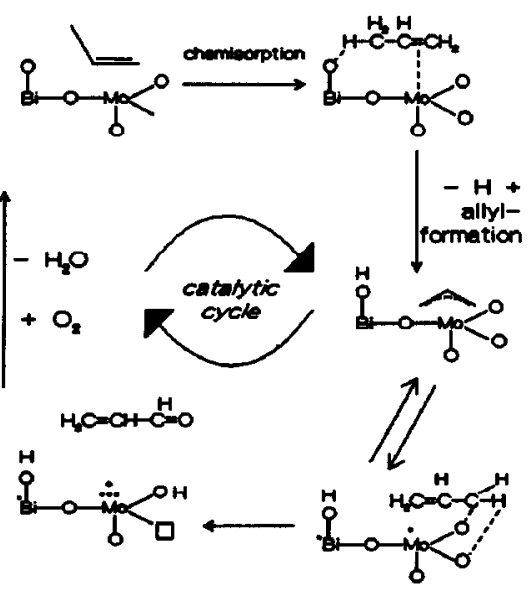

Fig. 27. Schematic mechanism of catalysis of olefin oxidation by bismuth molybdate type catalyst.

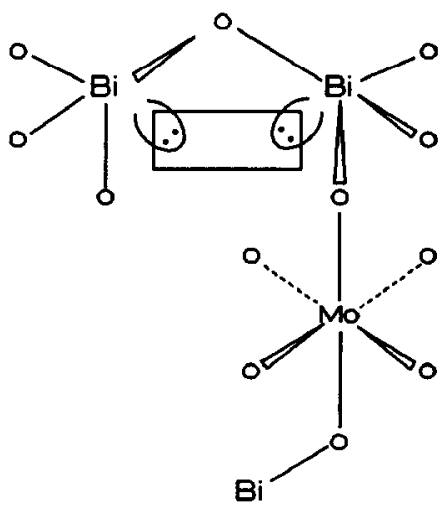

Fig. 28. Proposed active-site structure on the surface of $\mathrm{Bi}_{2} \mathrm{MoO}_{6}$ (see Ref. 308). 
A schematic representation of the mechanism is given in Fig. 27. This is in fact a simplified version adapted from the more complete catalytic cycles proposed by Grasselli et al. $[305,307]$ for the oxidation and ammoxidation of olefins with bismuth molybdate and uranium antimonate catalysts.

Glaeser et al. [308] have shown in a recent investigation of propene oxidation on $\mathrm{Bi}_{2} \mathrm{MoO}_{6}$, by means of in situ Raman spectroscopy and reoxidation with ${ }^{18} \mathrm{O}_{2}$, together with pulse experiments, that movement of oxygen ions through the lattice is the rate limiting step in catalyst reoxidation. This is in agreement with the fact that these mixed oxides show ion conductivity [106 - 108], and with results published in many of the older papers on this subject. Furthermore they also show the multifunctional nature of the catalyst active site for propene oxidation. The $\alpha-\mathrm{H}$ abstraction occurs by an oxygen ion bridging bismuth and molybdenum ions while oxygen insertion occurs at centres associated with molybdenum as indicated schematically in Fig. 27. Sites for $\mathrm{O}_{2}$ chemisorption and reduction and dissociation are probably associated with the directed lone-pairs of electrons in the Bi-O-Bi species as shown schematically in Fig. 28.

As proposed by Grasselli et al. [305, 307, 309] there are a number of functions which must be simultaneously fulfilled by a catalyst in selective oxidation and in general different components are necessary for this. These components are indicated schematically in Table $10[307,309]$.

Table 10

Electronic structure of some components of selective oxidation catalysts

\begin{tabular}{|c|c|c|c|}
\hline$\alpha-\mathbf{H}$ abstraction & $\begin{array}{l}\text { olefin } \\
\text { chemisorption/ } \\
\text { O-insertion }\end{array}$ & $\begin{array}{l}\text { Redox } \\
\text { couple }\end{array}$ & Example \\
\hline \multirow[t]{2}{*}{$B i^{3+} 5 d^{10} 6 s^{2} 6 p^{0}$} & $\mathrm{Mo}^{6+} 4 \mathrm{~d}^{0} 5 \mathrm{~s}^{0}$ & $\mathrm{Ce}^{3+} / \mathrm{Ce}^{4+}$ & $\mathrm{Bi}_{2} \mathrm{O}_{3} \cdot \mathrm{nMoO}_{3}$ \\
\hline & & $\mathrm{Fe}^{2+} / \mathrm{Fe}^{3+}$ & $\mathrm{M}_{\mathrm{a}}^{2+} \mathrm{M}_{\mathrm{b}}{ }^{3+} \mathrm{Bi}_{\mathrm{x}} \mathrm{Mo}_{\mathrm{y}} \mathrm{O}_{\mathrm{z}}$ \\
\hline \multirow[t]{2}{*}{$T e^{4+} 4 d^{10} 5 s^{2} 5 p^{0}$} & $\mathrm{Mo}^{6+} 4 \mathrm{~d}^{0} 5 \mathrm{~s}^{0}$ & $\mathrm{Ce}^{3+} / \mathrm{Ce}^{4+}$ & $\mathrm{Te}_{2} \mathrm{MoO}_{7}$ \\
\hline & & & $\left(\mathrm{Te}_{\mathrm{a}} \mathrm{Ce}_{\mathrm{b}} \mathrm{Mo}_{\mathrm{y}}\right) \mathrm{O}_{\mathrm{z}}$ \\
\hline $\mathrm{Sb}^{3+} 4 \mathrm{~d}^{10} 5 \mathrm{~s}^{2} 5 \mathrm{p}^{0}$ & $S b^{5+} 5 s^{0} 5 d^{0}$ & $\mathrm{Fe}^{2+} / \mathrm{Fe}^{3+}$ & $\mathrm{Fe}_{\mathbf{x}} \mathrm{Sb}_{\mathbf{y}} \mathrm{O}_{z}$ \\
\hline$U^{5+} 5 f^{1} 6 d^{0} 7 s^{0}$ & $\mathrm{Sb}^{5+} 5 \mathrm{~s}^{0} 5 \mathrm{~d}^{0}$ & $U^{5+} / U^{6+}$ & $\mathrm{USb}_{3} \mathrm{O}_{10}$ \\
\hline $\mathrm{Se}^{4+} 3 \mathrm{~d}^{10} 4 \mathrm{~s}^{2} 4 \mathrm{p}^{0}$ & $\mathrm{Te}^{6+} 5 \mathrm{~s}^{0} 5 \mathrm{p}^{0}$ & $\mathrm{Fe}^{2+} / \mathrm{Fe}^{3+}$ & $\mathrm{Fe}_{\mathrm{a}} \mathrm{Se}_{\mathrm{b}}{ }^{4+} \mathrm{Te}_{\mathrm{c}}{ }^{6+} \mathrm{O}_{\mathrm{x}}$ \\
\hline
\end{tabular}


The $\alpha-\mathrm{H}$ abstracting element generally requires a lone pair of electrons or some electron density on the metal ion. The olefin adsorption and $\mathrm{O}$ (or $\mathrm{NH}$ ) insertion components require coordinative unsaturation and vacant orbitals, both for chemisorption and for electron transfer. The redox couples consist of multivalent elements in close structural proximity to the previously mentioned groups. These must promote oxygen ion transport, stabilize oxygen deficient structures and must also maintain the active sites in a high oxidation state. An interesting application of these ideas has been proposed by Brazdil et al. [25] (see also Refs. 305, 307, 309) for bismuth-cerium molybdate catalysts. The compounds $\mathrm{Bi}_{2-\mathrm{x}} \mathrm{Ce}_{\mathrm{x}} \mathrm{Mo}_{3} \mathrm{O}_{12}$ form a two phase system, with a homogeneous solid solution of cerium in $\mathrm{Bi}_{2} \mathrm{Mo}_{3} \mathrm{O}_{12}$ up to $\mathrm{x} \approx 0.25$ and a homogeneous solid solution of bismuth in $\mathrm{Ce}_{2} \mathrm{Mo}_{3} \mathrm{O}_{12}$ above $\mathrm{x} \approx 1$ to 1.25 . This system shows maximum activity for propene (amm)oxidation just at the boundary compositions between the single phase and two phase regions and approximately in the middle of the two phase region. The authors propose that optimum catalytic activity is obtained when there is a maximum interaction between the active components: this occurs in single phase solid solutions and the amounts, within one phase, are at a maximum near the phase boundary. The maximum in the middle of the two phase region is connected with the fact that the crystal structures are similar and coherent phase boundaries can be formed then. This is important because oxygen ion and electron transfer between the two phases will be easiest if the mismatch between their structures is as small as possible.

In a recent, careful kinetic study of the partial oxidation of 1-butene to 1,3-butadiene over bismuth molybdate catalysts by Burban et al. [310, 311] the authors could show that the general ideas of the mechanism proposed by Glaeser et al. [308] and Grasselli et al. [304, 305] are correct. In particular the importance of oxygen and charge transport through the bulk of the catalyst was substantiated.

The influence of defect properties on the sensor behaviour of bismuth molybdate is discussed by Hykaway et al. [312] who show that the fast response to changes in gas composition are clearly related with the high ion conductivity of this type of compound. The importance of the surface reactions is indicated by the observation that the sensitivity towards alkenes, alcohols and ketones is high whereas that towards alkanes is very low, which is also in agreement with the catalytic behaviour of these materials. Sears [313] has also shown that bismuth-iron molybdate catalysts act as sensors for reducing vapors by bulk conductivity changes due to the change in oxygen vacancy concentration. These vacancies are very mobile and diffuse quickly through the material.

Sears [314] recently discussed the use of bismuth molybdates and bismuth iron molybdates as gas sensors with a high sensitivity to alcohols and ketones. The mechanism of the sensors is that, due to the reduction of the catalyst, highly mobile oxygen vacancies are formed which diffuse quickly through the material and through their effect on the electronic carrier density lead to changes in conductance. The sensitivity for hydrocarbons, carbon monoxide and hydrogen is low, but for alcohols and ketones much 
higher, which is clearly related to the catalytic activity for oxidation of these compounds.

Crystallographic effects on the selective oxidation of propene with bismuth molybdate catalysts were considered by Theobald et al. [315]. They observed, in agreement with results published by others, that $\alpha-\mathrm{Bi}_{2} \mathrm{Mo}_{3} \mathrm{O}_{12}$ is much more active than $r-\mathrm{Bi}_{2} \mathrm{MoO}_{6}$. This was ascribed to the fact that both bismuth and molybdenum ions must be present in the surface for this reaction, as indicated, e.g., by Grasselli and Burrington [304]. The $\alpha$-bismuth molybdate has the (010) plane, containing both bismuth and molybdenum ions, as main cleavage plane. The $r$-bismuth molybdate, on the other hand, only has both ions present on small lateral faces and not on the main cleavage plane.

The fact that the selectivity is also higher on the $\alpha$-bismuth molybdate than on the $\gamma$-compound is, according to these authors, due to a smaller value of the ion conductivity as a consequence of a special orientation of the bismuth lone pairs in the lattice. This explanation is derived from a comparison of the results obtained with $\mathrm{Bi}_{2} \mathrm{MoO}_{6}$ and $\mathrm{Sb}_{2} \mathrm{MoO}_{6}$. The latter shows an activity for acrolein formation which is half that of the bismuth compound, but the activity for $\mathrm{CO}_{2}$ formation is 20 times lower. Thus the lower conductivity has a larger influence on the total oxidation than on the selective oxidation.

Hayakawa et al. [316] studied propene oxidation on bismuth molybdate catalysts by means of a solid electrolyte electrochemical cell, in which the oxygen was supplied through the yttria stabilized zirconia solid electrolyte, while the catalyst was present as a thin layer on one of the electrodes. In this investigation $\mathrm{MoO}_{3}, \alpha-\mathrm{Bi}_{2} \mathrm{Mo}_{3} \mathrm{O}_{12}$, $\beta-\mathrm{Bi}_{2} \mathrm{Mo}_{2} \mathrm{O}_{9}, \gamma-\mathrm{Bi}_{2} \mathrm{MoO}_{6}$ and $\mathrm{Bi}_{2} \mathrm{O}_{3}$ were used as catalysts. In the case of the ordinary gas phase reaction the first two compounds and $\mathrm{Bi}_{2} \mathrm{O}_{3}$ show hardly any catalytic activity for propene oxidation and only $\beta$ and $\gamma$ bismuth molybdate are active. In the case of the electrochemical cell $\mathrm{MoO}_{3}$ and all bismuth molybdates show roughly the same, high activity while $\mathrm{Bi}_{2} \mathrm{O}_{3}$ remains inactive. This supports the idea that oxygen atoms bound to molybdenum insert into an allylic intermediate of propene, while bismuth bound species are responsible for the reoxidation of the catalyst and where oxygen transport through the catalyst is a necessary step.

The role of bismuth and of cation vacancies in substituted scheelite catalysts for the selective oxidation and ammoxidation of propene has been elucidated by Brazdil et al. [303]. The investigated compounds were all derived from $\mathrm{PbMoO}_{4}$, which has the scheelite structure. From infra red spectroscopic studies, together with kinetic investigation, it was concluded that bismuth-oxygen groups, and not cation vacancies, are responsible for the formation of the allylic intermediate by proton abstraction from propene. The cation vacancies were shown to form Mo-O centers on which the olefin is adsorbed and by which oxygen, or in ammoxidation nitrogen, is inserted in the allytlic intermediate. Furthermore the cation vacancies increase the diffusion of oxygen through the lattice and facilitate the replenishment of oxygen ions in the catalytic cycle. They also maintain the oxygen insertion Mo-O centers in the optimum oxidation state. These results are in agreement with the ideas about the mechanism of oxidation on bismuth molybdate 
catalysts as discussed above and by Grasselli et al. [317].

Ueda et al. [318] studied the oxidation of propene by tricomponent metal oxides of the type $\mathrm{Bi}_{1-\mathrm{x} / 3} \mathrm{~V}_{1-\mathrm{x}} \mathrm{Mo}_{\mathrm{x}} \mathrm{O}_{4}$ with the scheelite structure. These authors observed that the kinetic parameters (reaction orders and activation enthalpy) did not change but that the activity, and thus the number of active sites, increased with increasing substitution of $\mathrm{V}^{5+}$ by $\mathrm{Mo}^{6+}$ up to about $\mathrm{x}=0.5$. From ${ }^{18} \mathrm{O}_{2}$ experiments it followed that only lattice oxygen atoms were incorporated in the acrolein formed in this reaction. An estimate of the mobility of the lattice oxide ions could be obtained from the ${ }^{18} \mathrm{O}_{2}$ tracer measurements. The results show a good agreement between the increase of the catalytic activity and the mobility of the lattice oxide ions. Unsubstituted scheelites do not show ion conductivity. The authors propose that the presence of oxygen vacancies, caused by the substitution of vanadium, lead to oxygen sharing between the $(\mathrm{V}, \mathrm{Mo}) \mathrm{O}_{4}$ tetrahedra and that in this way migration of oxygen becomes possible by exchange of shared oxygens between tetrahedra, which is not possible between the complete and separate tetrahedra in the pure scheelite structure.

In a study of catalysis by scheelite-type oxides $\mathrm{Pb}_{1-3 \mathrm{x}} \mathrm{Bi}_{2 \mathrm{x}} \Phi_{\mathrm{x}} \mathrm{MoO}_{4}(\Phi=$ cation vacancy) for the oxidation of alkenes in an electrochemical reactor, Hayakawa et al. [319] used a solid electrolyte electrochemical cell with the scheelite as a thin layer on a gold anode. Comparing the reactions where oxygen is pumped through the electrolyte and those where it is present in the gas phase together with the alkene, these authors conclude that oxygen from the bulk inserts into the allylic intermediate in the formation of the oxygenated product. Oxygen species chemisorbed on the catalyst surface from the gas phase are responsible for the catalysis of complete combustion.

As mentioned earlier Yamazoe and Teraoka [265] have published a survey of studies concerned with the application of perovskite type materials as catalysts, among others for the selective oxidation of hydrocarbons such as methane, propene, propane, i-butene, toluene and also of methanol.

\subsection{Oxidative dehydrogenation and dehydrodimerization}

Di Cosimo et al. [258] studied the oxidative dehydrodimerization of propene at $600{ }^{\circ} \mathrm{C}$ using $\left(\mathrm{Bi}_{2} \mathrm{O}_{3}\right)_{0.85}\left(\mathrm{La}_{2} \mathrm{O}_{3}\right)_{0.15}$ as catalyst membrane separating a feed of $20 \%$ propene in helium on one side from air on the other side. This means that the catalyst after reduction by the propene is reoxidized by oxygen transported through the solid due to the chemical potential gradient of oxygen on both sides of the membrane. The selectivity and the yield obtained in this way (yield of hexadiene and benzene of $2.5 \%$ ) was found to be significantly larger than if oxygen was directly fed to the propene-helium mixture. In the latter case the yield of hexadiene and benzene was $1.4-1.7 \%$. These results support the idea that in the selective oxidation of propene the lattice oxygen is predominantly involved. Another important conclusion of this work is that, in these catalysts based on bismuth oxide, reoxidation can occur by means of oxygen conduction 
in the mixed conductor. In this way the catalytic reaction is completely separated from the regeneration of the catalyst.

Che and Tench [142] present evidence that in some cases adsorbed oxygen also plays a role and the reactions are then not limited to lattice oxygen. Sancier et al. [320] performed oxidation of propene over bismuth molybdate using ${ }^{18} \mathrm{O}_{2}$ at low temperatures. In contrast to the results of Keulks [321], obtained at higher temperatures, they observed that the acrolein formed contained ${ }^{18} \mathrm{O}$ and not only ${ }^{16} \mathrm{O}$ from the bismuth molybdate lattice. This means that at lower temperatures this oxidation must involve some form of adsorbed oxygen, although its nature is not clear. More extensive measurements by Keulks and Krenzke [322] indicate that indeed only lattice oxygen is used in the oxidation of propene. The participation of lattice oxygen in catalytic oxidation is further discussed in Section 13.2.

Centi and Trifiro [323] recently reviewed the selective oxidation, in particular the oxidative dehydrogenation, of olefins by antimony containing catalysts with the emphasis on mixed oxides with rutile-type structures. Contrary to what is observed in pure antimony oxides, which can only be reduced but not directly reoxidized, reoxidation after reduction is possible in the rutile-type catalysts and also in antimonates with the related trirutile structure. The authors suggest that these enhanced redox properties may be related to characteristics of the rutile-type matrix, e.g., fast ionic diffusion or formation of crystallographic shear planes. The importance of ionic diffusion in these catalysts is also shown by the observation that excess antimony inside the rutile-type structure takes part in the reaction in a reducing atmosphere, but not in an oxidizing one. As noted earlier (see Section 7.2 and Ref. 127) it is also pointed out by Centi and Trifiro that segregation and the difference between bulk and surface composition of these catalysts is very important.

The oxidative dehydrogenation of butene by a number of bismuth molybdate catalysts has been investigated by van Oeffelen et al. [324]. In this study the catalytic reaction was performed both in a continuous flow system and by sending pulses of butene over the catalyst. During the pulse experiments in situ measurement of the electrical conductivity was performed on $\mathrm{MoO}_{3}, \mathrm{Bi}_{2} \mathrm{Mo}_{2} \mathrm{O}_{9}$ and $\mathrm{Bi}_{2} \mathrm{MoO}_{6}$. XPS measurements showed that, in particular after reduction of the materials by butene, the $\mathrm{Bi} / \mathrm{Mo}$ ratio was much lower than in the bulk. The authors propose as the reason for this that part of the bismuth is reduced to metal and evaporates from the catalyst.

While pulsing butene over the catalysts the conductivity decreased, while it increased again when $\mathrm{O}_{2}$ was admitted to the catalyst. After a number of pulses, depending on the composition of the catalyst, the conductivity became constant even though reduction still proceeded. The constant conductivity was explained by proposing that in that case the migration of oxygen ions from the bulk to the surface (or from oxygen vacancies into the oxide) is fast enough to replenish the oxygen reacting with butene at the surface. Because, together with the oxygen, due to the electroneutrality 
condition, electron migration must also occur, the electrons migrate inwards. In the bulk they evidently do not contribute any further to the measured conductivity. The increased conductivity thus is mainly a surface effect. This is one of the very few instances where the effect of surface charges and electric fields, discussed in Section 7.3, have been applied to a specific catalytic process.

Using band model considerations the authors were also able to give a qualitative explanation for the model, mentioned in Section 7.3 in which reoxidation of these catalysts is a process which involves dissociative chemisorption of oxygen at sites on the catalyst surface other than those where the organic molecule reacts.

\subsection{Oxidative coupling of methane}

An interesting reaction which is widely investigated at present is the oxidative coupling of methane to form higher hydrocarbons, such as ethane and ethene, see e.g. Refs. 325, 326.

This reaction has been studied at $973 \mathrm{~K}$ using many different oxide systems by Otsuka et al. [327, 328] who observed that $\mathrm{Sm}_{2} \mathrm{O}_{3}$ and $\mathrm{Bi}_{2} \mathrm{O}_{3}$ are the most active catalysts for the reaction under consideration at the measuring temperature. As this is below the transformation temperature of $\mathrm{Bi}_{2} \mathrm{O}_{3}$ to the $\delta$ form this oxide is a mixed conductor under the reaction conditions. Balakireva et al. [329] studied the conducting properties of lanthanide oxides including $\mathrm{Sm}_{2} \mathrm{O}_{3}$. This is a p-type conductor when pure and, when doped with $\mathrm{CaO}$, the p-type conductivity increases and it becomes an ion conductor. As the purity of the $\mathrm{Sm}_{2} \mathrm{O}_{3}$ used by Otsuka et al. [328] is not known, definite conclusions concerning the influence of the conducting properties under the reaction conditions are not possible.

The kinetics of the methane coupling reaction has been studied by several authors $[325,326,330]$. In general this reaction is assumed to start with the abstraction of a hydrogen atom under formation of a methyl radical, which then dimerizes to $\mathrm{C}_{2} \mathrm{H}_{6}$. This is followed by oxidative dehydrogenation on the catalyst to $\mathrm{C}_{2} \mathrm{H}_{4}$. The main products which lower the selectivity are $\mathrm{CO}$ and $\mathrm{CO}_{2}$ and these are also thought to be formed under the influence of the catalyst, which thus should only be active in proton abstraction but not in oxidation.

Thomas et al. [331] studied a new family of catalysts for methane coupling, namely oxychlorides of bismuth. These compounds are structurally related to the bismuth molybdates and have a similar layer structure, with $\left[\mathrm{Bi}_{2} \mathrm{O}_{2}\right]^{2+}$ layers as shown in Fig. 12 which are observed to be among the most effective catalysts for this reaction. It could not be ascertained whether oxygen from the lattice is involved in the reaction, which on these catalysts seems to follow the same mechanism as discussed above for oxidic catalysts. On the basis of the general properties of these compounds the authors propose the study of fluorites and pyrochlores as promising catalysts for methane coupling.

The oxidative dimerization of methane on cerium mixed oxides was also studied 
by Machida and Enyo [332] who observe an increased selectivity toward $C_{2}$ products on compounds of the type $\mathrm{SrCe}_{0.9} \mathrm{Yb}_{0.1} \mathrm{O}_{2.95}$ and a decreased selectivity on $\mathrm{Ce}_{0.95} \mathrm{Yb}_{0.1} \mathrm{O}_{1.95}$. This is ascribed by the authors to the fact that the first compound is a proton conductor, whereas the second is an oxygen ion conductor. This is based on the idea that the first intermediate on the surface, probably a methyl radical, may be rapidly oxidized further to $\mathrm{CO}$ or $\mathrm{CO}_{2}$ if oxygen can be supplied from the lattice, but this cannot occur on a proton conducting material. An interesting application of proton conducting oxides, which falls outside the scope of this review, has been described by Woldman and Sokolovskii [333]. They use $\mathrm{SrO}\left(\mathrm{CeO}_{2}\right)_{0.95}\left(\mathrm{YbO}_{1.5}\right)_{0.05}$ as a proton conducting solid electrolyte with $\mathrm{La}_{0.6} \mathrm{Sr}_{0.4} \mathrm{MnO}_{3}$ as an anode in a cell for the electrolytic reaction of methane. At zero voltage a current is generated when methane is present in the anode compartment. This offers possibilities both for the electrocatalytic conversion of methane and for the development of a methane sensor. Chiang et al. [334] studied the electrocatalytic, nonoxidative dimerization of methane using an electrochemical cell with silver electrodes and an electrolyte of the same type as used by Machido and Enyo [332] and by Woldman and Sokolovskii [333]: $\mathrm{Ag} / \mathrm{SrCe}_{0.95} \mathrm{Yb}_{0.05} \mathrm{O}_{3-\alpha} / \mathrm{Ag}$. Chiang et al. observe that the rate of methane dehydrogenation to ethane and ethene can increase up to eight times the open circuit rate. This indicates that the activity and selectivity of the silver electrodes are significantly influenced upon passing current through the cell. This is probably caused by the changed properties of hydrogen on the silver surface.

\subsection{Hydrocarbon combustion}

Catalytic combustion offers several advantages over conventional combustion, such as lower emission levels of contaminants such as nitrogen oxides, better fuel efficiency and lower local peak temperatures. On the other hand, the materials to be used as catalysts must meet the simultaneous requirements of extreme thermal and chemical stability and high activity in the high oxygen, high temperature combustion environment. In particular complex multicomponent metal oxides, such as the perovskites [11, 273], have been proposed as being suitable because they combine low volatility, relatively high catalytic activity and reasonable resistance against sintering. A compilation of perovskite type materials used as catalysts, among other things, for hydrocarbon combustion has recently been given by Yamazoe and Teraoka [265].

Arai et al. [335] studied the catalytic combustion of methane over various perovskite type oxides as catalysts. The perovskites studied were $\mathrm{La}_{1-x} \mathrm{~A}_{\mathbf{x}} \mathrm{BO}_{4}$ with $\mathrm{A}=$ $\mathrm{Ca}, \mathrm{Sr}, \mathrm{Ba}$ and $\mathrm{Ce}$ and $\mathrm{B}=\mathrm{Co}, \mathrm{Mn}, \mathrm{Cu}, \mathrm{Ni}$ and $\mathrm{Fe}$. When $\mathrm{x}=0$ the most active catalysts were those with $\mathrm{B}=\mathrm{Co}, \mathrm{Mn}$ and $\mathrm{Fe}$. When A-type substitution was applied, which means introducing oxygen vacancies and increasing ionic conductivity, the activities increased and the most active catalyst was $\mathrm{La}_{0.6} \mathrm{Sr}_{0.4} \mathrm{MnO}_{3-\delta}$, being nearly as active as platinum on $\gamma-\mathrm{Al}_{2} \mathrm{O}_{3}$. From the kinetics and the temperature dependence of the reaction rate it could be concluded that at relatively low temperatures $(<775 \mathrm{~K})$ adsorbed oxygen was 
responsible for the oxidation. At higher temperatures the adsorbed oxygen desorbs and increasing amounts of lattice oxygen take part in the reaction. The kinetics is described by an equation of the form:

rate $=\mathrm{k}_{1} \times \mathrm{P}_{\mathrm{CH}_{4}}+\mathrm{k}_{2} \times \mathrm{PCH}_{4} \times \mathrm{PO}_{2}^{1 / 2}$

where the first term, which is independent of the external oxygen partial pressure, is due to the reaction with lattice oxygen and the second term represents the reaction with atomically adsorbed oxygen (compare also Eqn. (60)).

These results indicate that at low temperatures the catalytic combustion of hydrocarbons proceeds according to a suprafacial mechanism, while at high temperatures an intrafacial mechanism is operative.

A similar investigation, with comparable results, has been described by McCarty and Wise [6] who, strangely enough, do not refer to the paper by Arai et al. [335]. These authors find the same kinetic equation as Arai et al. and similar kinetic parameters. As indicated by McCarty and Wise the electrons which arise from the removal of oxygen ions can lead to reduction of the transition metal ions. This means that in the steady-state hydrocarbon oxidation a type of redox mechanism is taking place. In this mechanism the anion vacancies, present in the lattice, are essential for oxygen adsorption, while the valence changes of the cations are necessary for the adsorption of the hydrocarbon species.

The increased concentration of the anion vacancies, due to the ongoing reaction, provides a driving force for oxygen adsorption. This explains the similarity in activity and selectivity of lattice and gas phase oxygen for a given catalyst. The type and valence of the transition metal cations, the metal to oxygen bond strength and the different degrees of covalency explain the variations in reactivity for different perovskites.

It is highly probable that the mixed conductivity of substituted perovskites described earlier [61, 95, 96] and the corresponding diffusive oxygen ion transport, are directly related with the catalytic processes. The activation enthalpies of the oxygen ion diffusion of $75 \pm 20 \mathrm{~kJ} \cdot \mathrm{mol}^{-1}$ lies very close to that for the catalytic combustion of methane, which is $70-80 \mathrm{~kJ}^{-\mathrm{mol}^{-1}}$. This strongly suggests that oxygen vacancies and their transport play a key role in the catalytic combustion reactions.

The combustion of n-butane and methane has been studied by Zhang et al. [149] using perovskites of the type $\mathrm{La}_{1-x} \mathrm{Sr}_{x} \mathrm{Co}_{1-y} \mathrm{Fe}_{y} \mathrm{O}_{3}$ as catalysts. In particular the purpose was to find a relation between the catalytic properties and the oxygen (de)sorption properties, which were discussed in Section 8.2. The authors observed a clear connection between the defect structure and the catalytic activity, which shows a maximum at $x \approx$ 0.2 for the pure cobalt compound and $x \approx 0.4$ for the compound with $y=0.4$. This is clearly due to a balance between two opposing effects: the amount of active oxygen increases with $x$, but its activity decreases.

The catalytic combustion of n-butane, which takes place at $200-300{ }^{\circ} \mathrm{C}$, increases 
with decreasing $y$ in $\mathrm{La}_{1-x} \mathrm{Sr}_{x} \mathrm{Co}_{1-y} \mathrm{Fe}_{y} \mathrm{O}_{3}$. This is also the direction in which the amount of oxygen available decreases. Methane combustion occurs at higher temperatures $\left(350-500^{\circ} \mathrm{C}\right)$ where the amount of available oxygen is nearly independent of the value of $y$. This explains why the activity for methane combustion also hardly changes with a change in $y$.

The chemisorption of oxygen and isobutene and the catalytic combustion of propene and isobutene were studied by Kremenic [336] on a series of perovskites $\mathrm{LaMO}_{3}$ with $\mathrm{M}=\mathrm{Cr}, \mathrm{Mn}, \mathrm{Fe}, \mathrm{Co}$ and $\mathrm{Ni}$. Both the adsorption of oxygen and the catalytic activity showed minima for $\mathrm{M}=\mathrm{Cr}, \mathrm{Fe}, \mathrm{Ni}$ and maxima for $\mathrm{Mn}$ and $\mathrm{Co}$. This may indicate an influence of the crystal field stabilization energies, although the peaks are shifted with respect to the expected positions. Isobutene combustion shows a maximum rate for iron with a monotonous decrease to chromium on one side and nickel on the other. Very limited partial oxidation products were found and only in a significant amount for the chromium compound. The clear dependence on the type of transition metal ion present points to the importance of localized interactions in these surface processes. This means that on these materials the combustion reactions show a suprafacial mechanism and that neither lattice oxygen nor electronic defects seem to be involved.

McCarty et al. [337] also reported the activity of some other perovskites, including the superconducting $\mathrm{YBa}_{2} \mathrm{Cu}_{3} \mathrm{O}_{7}$ and the layered perovskite $\mathrm{La}_{2} \mathrm{NiO}_{4}$. The same kinetics as described above (see Eqn. (63)) were observed in this investigation. The ability of lattice oxygen to oxidize methane was observed to correlate with the inverse of the stability of the complex oxide, within one class. Either the structure, but more probably the anion mobility, is an important factor in the combustion reaction in view of the much larger activity of the lanthanum perovskites compared with that of the alumina spinels. At sufficiently high temperatures $\left(1100 \mathrm{~K}\right.$ for $\left.\mathrm{LaFeO}_{3}\right)$ the oxidation rate becomes independent of the oxygen partial pressure. Under these conditions oxygen is readily incorporated or removed from perovskites, thus the supply of oxygen to the surface is insured when the rate of transport to the surface exceeds the combustion rate.

In their study of the defect structure of $\mathrm{La}_{1-\mathrm{x}} \mathrm{Sr}_{\mathbf{x}} \mathrm{CoO}_{3}$ Nakamura et al. [287] also investigated the activity of these compounds as catalysts for the combustion of propane and methanol. The reducibility, or in other words the oxidizing power, of the perovskites increased with increasing $x$, but the reoxidation became much slower. This explains that for low $\mathrm{x}$ the total activity increases, but decreases again at higher $\mathrm{x}$, when it is assumed that this reaction shows a reduction oxidation (Mars - van Krevelen) mechanism. This is also in agreement with the change in oxidation state of the catalyst as a function of $\mathrm{x}$ and of the $\mathrm{CO} / \mathrm{O}_{2}$ ratio.

Another example of the total oxidation of a hydrocarbon in a catalytic reaction is the total combustion of methane as fuel in a fuel cell studied by Steele et al. [338]. In this investigation it is shown that several oxide electrode materials, such as 
$\mathrm{Bi}_{2} \mathrm{O}_{3}-\mathrm{Pr}_{6} \mathrm{O}_{11}$, are much more active than platinum in the total oxidation of $\mathrm{CH}_{4}$ to carbon dioxide and $\mathrm{H}_{2} \mathrm{O}$. Similarly Mogensen and Bentzen [339] showed that a mixture of gadolinium doped $\mathrm{CeO}_{2}$ with yttria stabilized zirconia has attractive properties as mixed conducting electrode material for $\mathrm{CH}_{4}$ oxidation in a solid oxide fuel cell.

\subsection{Carbon dioxide splitting}

Another interesting reaction is the splitting of carbon dioxide according to:

$2 \mathrm{CO}_{2} \rightarrow 2 \mathrm{CO}+\mathrm{O}_{2}$

If this reaction is performed with a semipermeable membrane consisting of a mixed conducting material the equilibrium, which is rather unfavorable normally, can be shifted to the right by removing the oxygen. This has been studied by Nigara and Cales [256] using calcia stabilized zirconia, as the semipermeable membrane at temperatures from 1700 to $1950 \mathrm{~K}$, and they demonstrated the possibility of this application. Using a thinner material and a larger surface area are two possibilities to increase production of carbon monoxide. However, the greatest gain can be expected by choosing a material with better semipermeable properties, in particular with a higher electronic conductivity than calcium stabilized $\mathrm{ZrO}_{2}$. The authors propose among others yttrium doped $\mathrm{ZrO}_{2}-\mathrm{CeO}_{2}$ solid solutions as a promising material for this type of application.

The electrocatalytic conversion of carbon dioxide to $\mathrm{CH}_{4}$ was studied in a solid electrolyte electrochemical cell with an oxygen ion-conducting electrolyte by Gür et al. [340]. The authors explain the hydrogenation of the carbonaceous intermediate, formed by the deoxygenation on the solid electrolyte, by reactions occurring on the platinum. Strictly speaking, this work falls outside the subject of this review, because metallic platinum electrodes are used as catalyst. On the other hand it is worth to be mentioned because it indicates how an interesting and useful combination of properties can lead to new applications. Also, as discussed in Section 12.10, it may well be that under certain circumstances the solid electrolyte makes a significant contribution to the catalytic reaction.

\subsection{Miscellaneous reactions}

A reaction mentioned earlier is the steam reforming of heavy oils and residues which was studied by Ovenston et al. [113] using RF heating of conducting catalysts. As shown by Mirza et al. [341] these catalysts show an increased selectivity towards higher production of olefins. This was ascribed by Ovenston et al. [113] to the mobility of electrons and ions in these catalysts, comparing this with the results of Chu and Rohr [290] in the reduction of nitrogen oxides without giving any details of a proposed mechanism.

Two non oxidation reactions in which propene is involved are the dimerization to hexadiene and the metathesis to ethene and 2-butene. It has been shown by Tsuda et al. $[342,343]$ that on $\mathrm{WO}_{3}$, which is an insulator, dimerization dominates, while on 
$\mathrm{ReO}_{3}$, which shows metallic conduction, the metathesis is predominant. Kosaka et al. [344] therefore studied the behaviour of propene, using the tungsten bronzes $\mathrm{Na}_{4} \mathrm{WO}_{3}$ as catalysts. In fact $\mathrm{NaWO}_{3}$ is isoelectronic with $\mathrm{ReO}_{3}$, each sodium atom donating one electron to the delocalized d-orbitals of the tungsten ions. In these compounds it is known that a transition from insulating to metallic conduction takes place around $x=0.25$. Indeed Kosaka et al. observed that at low values of $x$ dimerization is the main reaction, while with increasing values of $x$ there is an increasing contribution of the metathesis reaction. This is further supported by the reduction of the catalyst: this causes an increase in the conductivity and in the contribution of the metathesis. As the authors indicate, oxygen vacancies produced by the reduction probably also play a role. Unfortunately no detailed mechanism is discussed, so the exact contribution of the conductivity and/or of the oxygen vacancies is not explained.

Another group of reactions for which the application of the type of catalysts considered here has been studied is the hydrogenation of carbon oxides. For perovskites this has been reviewed by Petunchi and Lombardo [345]. The main conclusion is that especially in $\mathrm{H}_{2}$ - $\mathrm{CO}$ (synthesis gas) reactions, the original perovskite has been reduced so much that the transition metal is (nearly) completely reduced to the metallic state. The lanthanide oxide is then left and in fact acts as a support for the metal particles. This means of course that there is no longer any direct relationship between the catalytic behaviour with the perovskite properties.

An interesting reaction is the isosynthesis reaction of carbon monoxide and hydrogen, i.e. the selective conversion of synthesis gas into branched aliphatic hydrocarbons, which has been studied extensively by Ekerdt et al. (e.g. Refs. 5, 346, 347). Oxygen labeling studies of this reaction [347] have shown that lattice oxygen is incorporated in methanol. This led to the proposal that in this reaction carbon monoxide reacts with $\mathrm{ZrO}_{2}$ to form a formate which is reduced to a methoxide. The latter is an intermediate in the reactions leading to methanol and to branched hydrocarbons. In a later study of $\mathrm{SO}_{3}$ and $\mathrm{CO}$ uptake [346] it was shown that $\mathrm{SO}_{3}$ is taken up by oxygen vacancies and forms a sulphate. Studying this on yttria doped zirconia showed that a maximum in the adsorption of both $\mathrm{SO}_{3}$ and carbon monoxide is observed at the yttria content corresponding with the highest ionic conductivity of yttria doped $\mathrm{ZrO}_{2}$. On the basis of the observation that the amount of formate or sulphate formed corresponds with the amount of methanol produced, it is concluded that surface oxygen ion vacancies are the catalytically active sites for activation of carbon monoxide and formation of methanol.

The chain growth in the isosynthesis reaction can occur by two mechanisms: by a CO insertion reaction or by a condensation reaction. Jackson and Ekerdt [5] used the isosynthesis profile, which is the ratio of $\mathrm{C}_{4}$ products to the sum of the $\mathrm{C}_{3}$ and $\mathrm{C}_{2}$ products, to determine the relative importance of the condensation reaction. It was shown that the isosynthesis profile is strongly influenced by the amount of dopant added to $\mathrm{ZrO}_{2}$. Both yttria and calcia stabilized zirconia catalysts showed a maximum isosynthesis 
profile and branched-to-linear ratio at the percentage stabilization corresponding to the maximum oxygen ion conductivity. It is suggested that the observed results are explained by a maximum availability of oxygen vacancies at the surface due to the high rate of vacancy migration.

The influence of other properties of the catalyst than the defect structure and conductivity is shown in the study by Jackson and Ekerdt [348] on the effect of the acidity of the zirconia. From isotope studies using labelled acetone and methanol it was shown that on acid modified zirconia branched $\mathrm{C}_{3}$ intermediates are more likely to be formed than linear $C_{3}$ intermediates. At the same time the amount of $C_{4}$ products increases with the acidity of the catalyst. It is clear from these observations that it is the balance of the different properties - defect structure, defect concentrations and acidity which determines the product distribution.

Trifird and Vaccari investigated the hydrogenation of carbon monoxide to methanol [349] using zinc chromates of the composition $\mathrm{Zn}_{1+\mathrm{x}} \mathrm{Cr}_{2-\mathrm{x}} \mathrm{O}_{4}$ as catalysts. The activity was clearly related to the nonstoichiometry of these compounds. Jackson et al. [350] showed, using computational modelling of different types of defects in zinc chromates, that the increase of the catalytic activity with the $\mathrm{Zn} / \mathrm{Cr}$ ratio is probably associated with an increasing concentration of oxygen vacancies.

Ryashentseva et al. [351] studied the activity of perovskite-type mixed rhenium oxides for the hydrogenation of benzene and ethyl acetate, together with the surface compositions of the catalysts both before and after use. The compounds investigated were of the general type $\mathrm{Ba}_{4-\mathrm{x}} \mathrm{Sr}_{\mathrm{x}} \mathrm{BRe}_{2} \Phi \mathrm{O}_{12}$, with $0 \leq \mathrm{x} \leq 4, \mathrm{~B}=\mathrm{Co}$, $\mathrm{Ni}$ or $\mathrm{Ca}$ and $\Phi=$ vacancy in the cationic lattice. The compounds with $\mathrm{x}=0$ are not active in hydrogenation and the activity increases with increasing $\mathrm{x}$ and also in the order $\mathrm{Ca}<\mathrm{Ni}<\mathrm{Co}$. The authors correlate this with the ionization energies of the elements, but do not give a direct relation with the mechanism of the catalytic reaction.

The bulk properties of the catalysts were not investigated. XPS investigation showed that, after use, a number of catalysts contain some metallic rhenium. It is suggested that the catalytic properties are nevertheless mainly determined by the perovskite part of the catalyst because the catalysts were still active, even when no metallic rhenium was formed. Phase analysis of the surfaces of $\mathrm{Sr}_{4} \mathrm{CoRe}_{2} \Phi \mathrm{O}_{12}$ and $\mathrm{Sr}_{4} \mathrm{NiRe}_{2} \Phi \mathrm{O}_{12}$, performed by means of thermovacuum conductivity measurements also showed that the semiconductor type of conductivity is retained, even when metallic rhenium is formed and that metallic conductivity is not observed. This means that the rhenium metal is only present in small, localized regions and does not dominate the catalytic behaviour.

In a similar investigation Nudel et al. [352] studied the hydrogenation of 1,3butadiene over the perovskite $\mathrm{LaCoO}_{3}$. This perovskite becomes active for this hydrogenation reaction after reduction by hydrogen at temperatures between 140 and 400 ${ }^{\circ} \mathrm{C}$, the catalytic activity increasing with reduction temperature. The experimental 
evidence indicates that metallic centres $\left(\mathrm{Co}^{9}\right)$ are formed during the reduction step and that these are the active sites for the hydrogenation reaction. The authors state that the rest of the surface, of which no composition or structure are given, also seems to play a role in the development of the catalytic activity. However, it is also stated to be certain that the reaction takes place on the metallic cobalt, while the rest of the oxide acts as a support. It is indicated that adsorption of 1,3-butadiene on the surface of the oxide may nevertheless play a role in the total reaction.

Hálasz [353] investigated the selective oxidation and dehydrogenation of methanol on $\mathrm{Y}-\mathrm{Ba}-\mathrm{Cu}-\mathrm{O}$ catalysts. The composition of the catalysts can be given as $\mathrm{YBa}_{2} \mathrm{Cu}_{3} \mathrm{O}_{7-\mathrm{x}}$ For the oxidized $(\mathrm{x}=0.1)$ or slightly reduced $(\mathrm{x}=0.5)$ form, in the presence of oxygen in the gas phase, only formaldehyde and water were formed. No metallic copper could be detected in the used catalyst. The only observable changes were that the perovskite had become tetragonal and that all copper was present as $\mathrm{Cu}^{2+}$, all $\mathrm{Cu}^{3+}$ having been reduced. When the reaction was performed around $600 \mathrm{~K}$ in the absence of oxygen, or when the catalyst was prereduced in hydrogen at $600 \mathrm{~K}$, the only products were methyl formate and water. After this treatment the catalysts contained metallic copper and the original perovskite had disappeared, being replaced by the perovskite $\mathrm{Y}_{2} \mathrm{BaCuO}_{5}, \mathrm{Y}_{2} \mathrm{O}_{3}$ and $\mathrm{BaCO}_{3}$. When this was reoxidized in oxygen formaldehyde and water were again the only products obtained from methanol.

Hálasz gives no indication as to the relation of the catalytic behaviour to the conducting properties of the original perovskite. It is suggested that the presence of $\mathrm{Cu}^{2+}$ is necessary for the formation of formaldehyde and that metallic copper catalyses the dehydrogenation with selective formation of methyl formate.

\subsection{Electrocatalytic phenomena}

As has been mentioned in the beginning this review is limited to the consideration of conducting, oxidic materials. On the other hand, many reactions have been studied on metallic electrodes during the last two decades, e.g. dissociation of $\mathrm{O}_{2}, \mathrm{NO}, \mathrm{CO}_{2}$, oxidation of $\mathrm{H}_{2}, \mathrm{NH}_{3}, \mathrm{CH}_{4}, \mathrm{CO}$, etc. Though different metal electrodes were applied in these studies, the results indicate in a number of cases the active role of the solid, oxide electrolyte itself in determining the net reaction rate under either cathodic or anodic polarization [234, 354, 355]. Reportedly high reactivity at high overpotentials has been explained by the creation of electronic defects, e.g., F-centers, at the oxide surface $[237,355,356]$.

Recently, Vayenas and co-workers [357, 358, 359] reported non-faradaic effects in electrocatalytic (partial) oxidation studies of several organic compounds in the sense that catalytic reaction rates exceed the rate of $\mathrm{O}^{2-}$-pumping up to a factor of $3 \times 10^{5}$. The effect, denoted by the acronym NEMCA, which stands for Non-faradaic Electrochemical Modification of Catalytic Activity, was not only observed using stabilized zirconia but also when $\boldsymbol{\beta}^{\prime \prime}-\mathrm{Al}_{2} \mathrm{O}_{3}$, which is a $\mathrm{Na}^{+}$conductor, was used as the solid 
electrolyte [360]. These phenomena have been attributed to changes in the work function of the catalyst electrode with applied electrical potential and concomitant changes in the strength of chemisorptive bonds [361].

Schouler [362] has given a survey of the relations which exist between the surface properties of solid oxide electrolytes and the kinetics of electrode reactions. In particular he has shown that in many cases the electrode polarization, if sufficiently high, leads to changes in the solid electrolyte. This becomes a mixed conductor due to the 'injection' of electrons or electron holes in the oxide. Typical electrode materials for which this this effect has been observed are $\left(\mathrm{ZrO}_{2}\right)_{1-x}\left(\mathrm{Y}_{2} \mathrm{O}_{3}\right)_{x}\left(\mathrm{ThO}_{2}\right)_{1-\mathrm{x}}\left(\mathrm{Y}_{2} \mathrm{O}_{3}\right)_{\mathrm{x}}$ and $\left(\mathrm{CeO}_{2}\right)_{1-\mathrm{x}}(\mathrm{CaO})_{x}$

For unpolarized electrodes there is in all cases a connection between the oxygen vacancy concentration and the electrode reaction rate. Although the exact mechanism has not yet been established, it is clear that in many cases electronic charge carriers participate in the reaction. This occurs even in materials like $\left(\mathrm{ZrO}_{2}\right)_{1 \cdot x}\left(\mathrm{Y}_{2} \mathrm{O}_{3}\right)_{x}$ which are known to have a very low electronic contribution to the total conductivity. These effects become more pronounced on sufficiently high polarization of the electrodes and thus are electrocatalytic effects. The basic idea is that at these polarization voltages intermediate ionized species are created and thus participate in the surface reactions.

In a later study [237] Schouler and Kleitz have shown that these electrocatalytic reactions can occur both at the anode and the cathode. From impedance spectroscopic measurements during steady-state polarization it could be concluded that the concentration polarization, which is commonly observed, is overcome by the formation of electronic defects in the solid electrolyte. These electrocatalytic effects are only due to a modification of the surface properties of the solid electrolyte and are independent of the electrode metal. These results confirm that the electrolyte surface does indeed contribute to the electrode reaction rate.

In principle the results of Hammouche et al. [101], which were discussed in Section 6, are similar to the effects discussed here. The main difference is that in that case the electrocatalytic effect consists in the formation of oxygen vacancies in an electronically conducting oxide. In fact there it is an electrocatalytic effect on an oxide electrode instead of on an oxide electrolyte as in the work of Schouler. 


\section{THE MECHANISM OF CATALYTIC OXIDATION IN PERSPECTIVE}

\subsection{General remarks}

From the foregoing it is seen that there seems to be a common background in a number of respects to catalytic oxidation, oxygen exchange, oxygen permeation, etc. Two types of behaviour are often distinguished in all these cases and all have to do with two possible ways in which oxygen reacts in these processes. Whether this is the separation into intrafacial and suprafacial reactions as proposed by Voorhoeve [11], that between electrophilic or nucleophilic oxygen as suggested by Bielanski and Haber [31], or the difference between homomolecular and heterogeneous oxygen exchange: in all these cases two possible oxygen reaction modes are distinguished.

In particular these indicate that oxygen may either react in the form of (relatively) loosely bound surface oxygen or as lattice oxygen. In particular, in the second type of reaction, the ion conducting properties of the oxide used as catalyst are probably of prime importance. Some ideas relevant to these considerations have also been discussed in connection with the relation between oxygen exchange and catalysis in Section 9.4.5.

Berkstresser et al. [276] have shown that the simultaneous presence of electronic defects and oxygen vacancies are very favourable in order to obtain a high catalytic activity.

\subsection{The participation of lattice exygen in catalytic reactions}

In several instances it was mentioned that lattice oxygen participates in catalytic reactions, indicating a connection between oxygen ion conduction and catalysis. Direct support for this idea comes, for example, from the work of Takasu et al. [146, 147] on carbon monoxide oxidation mentioned in Section 12.2. In the oxidation of NO Takasu et al. [145] have shown that it is highly probable that in this reaction too lattice oxygen is responsible for the oxidation of the NO.

As mentioned earlier Keulks and Krenzke [322] studied the role of lattice oxygen in the selective and total oxidation of propene. These authors also used ${ }^{18} \mathrm{O}_{2}$ in the gas phase and, from the slow increase in ${ }^{18} \mathrm{O}$ content in the oxidation products, concluded that the lattice oxygen of nearly the whole bulk of the catalyst is involved in this oxidation. In the carbon dioxide, produced by total oxidation, it is the lattice oxygen that participates, too. At the same time no exchange of the gaseous oxygen with the oxygen in the catalyst occurs, as shown by the constant ${ }^{18} \mathrm{O}$-content of the gas phase.

A general investigation of the involvement of lattice oxygen in the oxidation of propene on several catalysts has been reported by Moro-Oka et al. [363] using a ${ }^{18} \mathrm{O}$ tracer method. Some of their results are summarized in Table 11, in which some of the results of Keulks and Krenzke are given too. The basis of the method used was the measurement of the amount of ${ }^{18} \mathrm{O}$ present in the oxidation products, when the oxidation was performed in a gas mixture of ${ }^{18} \mathrm{O}_{2}$ and propene. 
Table 11

Number of layers of lattice oxygen involved in the oxidation of propene to acrolein

\begin{tabular}{|c|c|c|c|}
\hline Catalyst & $\begin{array}{l}\text { Temp } \\
\left({ }^{\circ} \mathrm{C}\right)\end{array}$ & $\begin{array}{l}\text { Number of } \\
\text { oxygen } \\
\text { layers }\end{array}$ & $\begin{array}{l}\text { Other catalysts } \\
\text { showing similar } \\
\text { results }\end{array}$ \\
\hline $\begin{array}{l}\mathrm{Fe}_{2} \mathrm{O}_{3}-\mathrm{Sb}_{2} \mathrm{O}_{4} \\
\mathrm{Fe} / \mathrm{Sb}=1: 4\end{array}$ & 450 & $6-7$ & $\begin{array}{l}\mathrm{M}-\mathrm{Sb}-\mathrm{O}(\mathrm{M}: \mathrm{Sb}=1: 4) \\
\mathrm{M}=\mathrm{Ni}, \mathrm{Co}, \mathrm{Mn}, \mathrm{Zn}\end{array}$ \\
\hline $1: 2$ & $\begin{array}{l}450 \\
350\end{array}$ & $\begin{array}{l}5 \\
3\end{array}$ & \\
\hline $1: 1$ & 450 & 4 & \\
\hline $4: 1$ & 350 & $0-2$ & \\
\hline $\mathrm{Sb}_{2} \mathrm{O}_{4}-\mathrm{MoO}_{3}(1: 1)$ & 450 & $20-40$ & \\
\hline $\mathrm{CoTeMoO}_{6}$ & 450 & $55-75$ & $\begin{array}{l}\mathrm{MnTeMoO}_{6}, \mathrm{TeO}_{2}- \\
\mathrm{MoO}_{3}(1: 1)\end{array}$ \\
\hline $\mathrm{Bi}_{2}\left(\mathrm{MoO}_{4}\right)_{3}$ & 430 & $75^{*}$ & \\
\hline $\mathrm{Bi}_{2} \mathrm{MoO}_{6}$ & 430 & $286^{*}$ & \\
\hline $\mathrm{Cu}_{2} \mathrm{O} /$ Celite & 260 & $0-1$ & \\
\hline $\mathrm{Co}_{17 / 18} \mathrm{Bi}_{1 / 18} \mathrm{MoO}_{4+\mathrm{x}}$ & 450 & $\begin{array}{l}\text { whole } \mathrm{Bi}_{2}\left(\mathrm{MoO}_{4}\right)_{3} \\
\text { phase }\end{array}$ & $\begin{array}{l}\mathrm{M}-\mathrm{Bi}-\mathrm{Mo}-\mathrm{O} \\
\mathrm{M}=\mathrm{Co}, \mathrm{Mn}, \mathrm{Mg}, \mathrm{Ni}\end{array}$ \\
\hline $\mathrm{Pb}_{11 / 12} \mathrm{Bi}_{1 / 12} \mathrm{MoO}_{4+x}$ & 450 & whole oxide & $\begin{array}{l}\mathrm{M}-\mathrm{Bi}-\mathrm{Mo}-\mathrm{O} \\
\mathrm{M}=\mathrm{Pb}, \mathrm{Ba}, \mathrm{Sr}\end{array}$ \\
\hline
\end{tabular}

* values taken from the data by Keulks and Krenzke [322].

It is seen that in the case of the iron oxide - antimony oxide catalysts and in that of $\mathrm{Cu}_{2} \mathrm{O}$ on Celite the depth of the oxide layer involved in the reaction is rather shallow, although in nearly all cases some oxygen from the bulk of the oxide certainly participates in the reactions. The different molybdenum containing oxides, on the other hand, clearly show that a large part, if not the whole of the oxide participates in the oxidation reaction. When it is assumed that there is complete equilibrium of the oxygen ions in the whole volume of the catalyst with that available for oxidation on the surface, uptake curves are calculated which agree reasonably well with the observed curves.

In the case of the compound $\mathrm{Pb}_{11 / 12} \mathrm{Bi}_{1 / 12} \mathrm{MoO}_{4+\mathrm{x}}$ this was found to be monophasic, thus being a homogeneous solid solution of bismuth and lead molybdates. Indeed $\mathrm{PbMoO}_{4}$ itself is inactive for the oxidation whereas the $\mathrm{Pb}-\mathrm{Bi}-\mathrm{Mo}-\mathrm{O}$ catalyst showed a high activity and selectivity in the reaction studied. This again indicates the important role of oxygen defects in these compounds. 
The study of the redox kinetics of bismuth molybdate catalysts by Brazdil et al. [364] also indicates the involvement of lattice oxygen in (amm)oxidation reactions. The catalysts investigated in that study were $\mathrm{Bi}_{2} \mathrm{Mo}_{3} \mathrm{O}_{12}, \mathrm{Bi}_{2} \mathrm{Mo}_{2} \mathrm{O}_{9}, \mathrm{Bi}_{2} \mathrm{MoO}_{6}$, $\mathrm{Bi}_{3} \mathrm{FeMO}_{2} \mathrm{O}_{12}$ and a multicomponent catalyst of the type $\mathrm{M}_{\mathrm{a}}{ }^{2+} \mathrm{M}_{\mathrm{b}}{ }^{3+} \mathrm{Bi}_{\mathrm{x}} \mathrm{Mo}_{\mathrm{y}} \mathrm{O}_{z}$ Using pulses of propene without oxygen it is observed that the multiphase catalyst gives an increasing yield and selectivity with increasing number of pulses. $\mathrm{Bi}_{2} \mathrm{Mo}_{3} \mathrm{O}_{12}$ and $\mathrm{Bi}_{2} \mathrm{Mo}_{2} \mathrm{O}_{9}$ also give maximum use of reactive lattice oxygen after partial reduction, while $\mathrm{Bi}_{2} \mathrm{MoO}_{6}$ and $\mathrm{Bi}_{3} \mathrm{FeMO}_{2} \mathrm{O}_{12}$ show maximum lattice oxygen activity in their highest oxidation state. Reoxidation rates of the partially reduced catalysts are first order in oxygen vacancy concentration and half order in gaseous oxygen and a general mechanism for this renxidation is proposed. All catalysts show two reoxidation regimes. The first has a low activation enthalpy and is connected with the reoxidation of surface vacancies. The second, with a much higher activation enthalpy corresponds with the reoxidation of oxygen vacancies in the bulk of the catalyst. Especially the latter is strongly dependent on the structure and the composition of the catalyst, as is to be expected because this will have a great influence on oxygen vacancy diffusion. 


\section{CONCLUDING REMARKS}

In this review clear evidence has been presented that in many catalytic oxidation reactions, using ion or mixed conducting oxides as catalysts, the conducting properties of these oxidic materials exert a great influence on their catalytic behaviour. In this respect the diffusivity of oxygen vacancies is of particular importance, but the electronic contribution to the total conductivity also plays a significant role. One of the important consequences of the oxygen ion mobility in these materials is that the reduction of the catalyst by the substrate to be oxidized can take place at different sites than those where the catalyst is reoxidized. This is especially favourable for selective oxidation because in this way the selectivity factor introduced by Callahan and Grasselli [270] (see Section 11.2) can more easily be met. By using catalytically active, porous or dense, membranes this makes the complete separation of the substrate and molecular oxygen possible.

An important point is also that the oxygen of the catalyst often performs different functions in the same reaction, proton abstraction and oxygen insertion being the most common. In general this means that different types of oxygen in, or adsorbed on, the catalyst, for example with different binding energies, must be available simultaneously.

Unfortunately only a limited number of systematic investigations have been performed on the correlation between the catalytic properties of the materials under consideration on the one hand, and their conducting properties on the other. Although the present authors are convinced that generalization to other materials and/or reactions of the results obtained by various investigators is valid, further research is needed to bridge the remaining gaps in the presently available knowledge.

From the preceding it will be clear that besides the interaction between mixed and ion conduction in oxides on the one hand and their catalytic behaviour on the other, other properties of the oxides can also have a significant influence which may, in certain cases, even overwhelm that of the conducting properties. Here further research is necessary too, to separate more clearly the influence of the different factors. Even in these cases it is to be expected that in the total mechanism the conducting properties of the catalyst still play an observable role, but more experimental evidence is very desirable.

A point of special importance is that the composition of the catalyst may be different at the surface from that of the bulk. This of course means that the conducting properties of the surface may also be different from that of the bulk, due to space charge and/or segregation effects. Of course it is the surface, with its special properties, which is directly involved in the catalytic reaction, meaning that the surface properties are of prime importance. It is to be expected that there exists a connection and correlation between surface and bulk properties. Thus there also is an urgent need for better knowledge concerning the relation between the conducting properties of the bulk and of the surface region in order to obtain a fuller understanding of the connection between 
these properties and the catalytic behaviour.

As mentioned in Section 11.2 it would also be very useful to devote more attention to the electronic structure of catalysts and catalyst surfaces by applying a molecular orbital analysis of atom superposition and electron delocalization. This may also throw more light on the special role of bismuth, in particular in the reoxidation of catalysts, which has been demonstrated experimentally, but is still not completely understood.

Another point, mentioned in Section 7.3, that merits further study for the catalyst materials considered in this review, is the influence of enhanced transport properties by surface induced effects.

\section{REFERENCES.}

1. T. Norby, Solid State Ionics $40 / 41$ (1990) 857.

2. M. Stoukides and C.G. Vayenas, J. Catal. 70 (1981) 137.

3. R.A. Goffe and D.M. Mason, J. Electrochem. Soc. 129 (1981) 447.

4. T.M. Gür and R.A. Huggins, J. Electrochem. Soc. 126 (1979) 1067.

5. N.B. Jackson and J.G. Ekerdt, J. Catal. 126 (1990) 31.

6. J.G. McCarty and H. Wise, Catalysis Today 8 (1990) 231.

7. P.J. Gellings, H.J.A. Koopmans, and A.J. Burggraaf, Applied Catalysis 39 (1988) 1.

8. C.G. Vayenas, Solid State Ionics $28-30$ (1988) 1521.

9. M.J. Verkerk, M.W.H. Hammink and A.J. Burggraaf, J. Electrochem. Soc. 130 (1983) 70.

10. J.M. Thomas, Solid State Ionics, $32 / 33$ (1989) 869.

11. R.J.H. Voorhoeve, Perovskite-Related Oxides as Oxidation-Reduction Catalysts, in: Advanced Materials in Catalysis, eds. J.J. Burton and R.L Garten, New York, Academic Press, 1977, p. 129 -180 .

12. A.W. Sleight, Crystal Chemistry and Catalytic Properties of Oxides with the Scheelite Structure, in: Advanced Materials in Catalysis, eds. J.J. Burton and R.L Garten, New York, Academic Press, 1977, p. 181 - 208.

13. B. Claudel, Adsorption and Catalysis on Ionic Solids, in: Electrode Processes in Solid State Ionics, eds. M. Kleitz and J. Dupuy, Dordrecht-Holland, D. Reidel, 1976, p. 45 - 81.

14. A. Cimino and S. Carra, in: Electrodes of conductive metallic oxides, Studies in Physical and Theoretical Chemistry, Vol. 11 (1980), Amsterdam, Elsevier, p. 97.

15. V.D. Sokolovskii, Catal. Rev.-Sci. Eng. 32 (1990) 1.

16. H.H. Kung, Transition Metal Oxides: Surface Chemistry and Catalysis, (Studies in Surface Science and Catalysis, Vol. 45), Amsterdam, Elsevier, 1989.

17. W.L. Worrell, Oxide solid electrolytes, in: Solid Elcctrolytes, ed. S. Geller, Berlin, Springer Verlag, Topics in Appl. Phys. 21 (1977) 143.

18. R.M. Dell and A. Hooper, Oxygen Ion Conductors, in: Solid Electrolytes, eds. P.Hagenmuller and W.van Gool, New York, Academic Press, 1978, p. 291.

19. E. Bergmann and H. Tannenberger, Interface Phenomena, in: Solid Electrolytes, eds. P.Hagenmuller and W.van Gool, New York, Academic Press, 1978, p. 173.

20. A.J. Burggraaf, B.A. Boukamp, I.C. Vinke, and K.J. de Vries, Recent developments in oxygen ion conducting solid electrolyte and electrode materials, in: Advances in Solid State Chemistry, vol II, ed. R. Catlow, Amsterdam, Elsevier, 1988, p.280.

21. T. Kudo and K. Fueki, Solid State Ionics, Tokyo, Kodansha/Weinheim, VCH Verlagsgesellschaft, 1990. 
22. M. Schiavello, La Chimica c MIndustria 61 (1979) 554.

23. Catalysis Today, vol. 8 (No. 2, 1990), eds. M. Misono and E.A. Lombardo.

24. Chemical and Physical Aspects of Catalytic Oxidation, eds. J.L. Portefaix and F. Figueras, Paris, Editions du CRNS, 1979.

25. J.F. Brazdil, R.G. Teller, R.K. Grasselli and E. Kostiner, Structural and Thermodynamic Basis for Catalytic Behavior of Bismuth-Cerium Molybdate Selective Oxidation Catalysts, in: Solid State Chemistry in Catalysis, eds. R.K. Grasselli and J.F. Brazdil, ACS Symposium Series 279, Washington D.C., American Chemical Society, 1985, p. 57.

26. F.S. Stone, Oxide Crystal Chemistry and Catalysis, in: Chemistry and Chemical Engineering of Catalytic Processes, eds. R. Prins and G.C.A. Schuit, Alphen aan den Rijn, Sijthoff \& Noordhoff, 1980, p. 477.

27. D. Barthomeuf and F. Figueras, Acid-base properties of oxidation catalysts, in: Chemical and Physical Aspects of Catalytic Oxidation, Eds J.L. Portefaix and F. Figueras, Paris, Editions du CRNS, 1979, 241.

28. C. Fouassier, Influence of Covalency on the Crystal Structure and Physical Properties of Oxides, in: Chemical and Physical Aspects of Catalytic Oxidation, Eds J.L. Portefaix and F. Figueras, Paris, Editions du CRNS, 1979, 271.

29. P.de Montgolfier, The Contribution of Theoretical Chemistry to the Understanding of Oxides and Adsorbed Molecules, in: Chemical and Physical Aspects of Catalytic Oxidation, Eds J.L. Portefaix and F. Figueras, Paris, Editions du CRNS, 1979, 291.

30. B.F. Claudel, Recent Developments of the Band Model in Chemisorption and Catalysis on Semi-Conductors, in: Chemical and Physical Aspects of Catalytic Oxidation, Eds J.L. Portefaix and F. Figueras, Paris, Editions du CRNS, 1979, p. 397.

31. A. Bielanski and J. Haber, Catal. Rev. - Sci. Eng. 19 (1979) 1.

32. K.R. Barnard, K. Foger, T.W. Turney, and R.D. Williams, J. Catal. 125 (1990) 265.

33. F.A. Kröger, The Chemistry of Imperfect Crystals, North Holland, Amsterdam, 2nd. ed. 1974.

34. L. Heyne, Electrochemistry of Mixed Ionic-Electronic Conductors, in: Solid Electrolytes, ed. S. Geller, Berlin, Springer Verlag, Topies in Appl. Phys. 21 (1977) 169.

35. H.L. Tuller, Mixed Conduction in Nonstoichiometric Oxides, in: "Nonstoichiometric Oxides", Ed. O.T. Sorrensen, New York, Academic Press, 1981, p. 271.

36. P. Kofstad, Non stoichiometry. Diffusion and Electrical Conductivity in Binary Metal Oxides, New York, Wiley/Interscience, 1972.

37. F.A. Kröger and H.J. Vink, Solid State Phys. 3 (1956) 307.

38. J.A. Kilner and B.C.H. Steele, Mass Transport in Anion-Deficient Fluorite Oxides, in: "Nonstoichiometric Oxides, ed. O.T. Sørensen, New York, Academic Press, 1981, p. 271.

39. D.M. Smyth, The Role of Impurities in Insulating Transition Metal Oxides, in: Progr. Solid St. Chem., 15 (1984) 145.

40. J.A.M. Van Roosmalen and E.H.P. Cordfunke, J. Solid State Chem. 93 (1991) 212.

41. G. Brouwer, Philips Res. Rept. 9 (1954) 366.

42. J.C. Rifflet, P. Odier, and A.M. Anthony, J. Am. Ceram. Soc. 58 (1975) 493.

43. P. Odier, J.C. Rifflet, and J.P. Loup, in: Proc. 9th Int.Symposium on the Reactivity of Solids, Cracow, sept. 1980, Amsterdam/ Warszawa, Elsevier/PWN, 1982, p. 458.

44. K.W. Browal and R.H. Doremus, J. Amer. Ceram. Soc. 60 (1977) 262

45. B. Cales and J.F. Baumard, Rev. Int. Hautes Temper. Refract. 17 (1980) 137

46. B. Cales and J.F. Baumard, J. Electrochem. Soc. 131 (1984) 2407

47. S.S. Liou and W.L. Worrell, Appl. Phys. A 49 (1989) 25.

48. W.L. Worrell and W. Weppner, Offenlegungschrift DE 3436597 A1 (1986)

49. T.A. Ramanarayanan, S. Ling, and M.P. Anderson, Proc. 2nd Int. Symp. Solid Oxide Fuel Cells, july 2 - 5, 1991, Athens, Greece 
50. J.W. Patterson, J. Electrochem. Soc. 118 (1971) 1033.

51. R.J. Borg and G.J. Dienes, An Introduction to Solid State Diffusion, Boston, Academic Press, 1988.

52. G.E. Murch, Solid State Ionics 6 (1982) 295.

53. A. Rouanet, Rev. Int. Hautes Tempér. et Réfract. 8 (1971) 161.

54. Phase Diagrams for Ceramists, G. Smith, editor, Vol. IV, 1981, Columbus, The American Ceramic Society.

55. V.S. Stubican, Adv. in Ceramics 241 (1988) 71.

56. Phase Diagrams for Ceramists, M.A. Clevinger and H.M. Ondik, editors, Vol. VI, 1987, Westerville, The American Ceramic Society.

57. H.A. Harwig, Z. anorg. allg. Chem. 444 (1978) 151.

58. H.A. Harwig and J.W. Weenk, Z. anorg. allg. Chem. 444 (1978) 167.

59. P.W.M. Jacobs, D.A. MacDonaill, and A.N. Cormack, in: Advances in Ceramies, Vol. 23: Non Stoichiometric compounds, eds. C.R.A. Catlow and W.C. Mackrodt, Westerville Ohio, American Ceramic Society, 1987, p. 307.

60. D.J. Buttrey, D.A. Jefferson, and J.M. Thomas, Phil. Mag. A, 53 (1986) 897.

61. Y. Teraoka, H.-M. Zhang, K. Okamoto, and N. Yamazoe, Mat. Res. Bull. 23 (1988) 51.

62. F.S. Galasso, Structure, Properties and Preparation of Perovskite-type Compounds, Oxford, Pergamon Press, 1969.

63. C.P. Khattak and F.F.Y. Wang, Perouskites and Garnets, in: Handbook on the Physics and Chemistry of the Rare Earths, Amsterdam, North Holland, 1979, Vol. 3, p. 525.

64. J.B. Goodenough and J.M. Longo, Crystallographic and Magnetic Properties of Perovskite and Perovskite-related Oxides, in: Landolt-Börnstein, New Series, Berlin, Springer Verlag, 1970, Group III, Vol. 4, p. 126.

65. E.J. Baran, Catalysis Today 8 (1990) 133.

66. C.N.R. Rao, J. Gopalakrishnan, and K. Vidyasagar, Indian J. Chem. 23A (1984) 265.

67. D.M. Smyth, Defects and Order in Perovskite-related Oxides, in: Ann. Rev. Mater. Sci. 15 (1985) 329.

68. L.G. Tejuca, J.L.G. Fierro, and J.M.D. Tascon, Structure and Reactivity of Perovskite-Type Oxides, in: Adv. Catal. 36 (1989) 237.

69. W.M.H. Sachtler and N.H. de Boer, Proc. 3rd Int. Congr. Catal., Amsterdam, North Holland, 1965, p. 252.

70. V.A. Roiter, G. Golodets and Yu. Pyatnitzkii, About the Use of Thermodynamics for Predicting Catalytic Action, in: Proc. 4th Internat. Congr. Catalysis (Moscow, 1968), Amsterdam, Elsevier, 1969, p. 466.

71. W.M.H. Sachtler, G.J.H. Dorgelo, J. Fahrenfort, and R.J.H. Voorhoeve, Correlations between Catalytic and Thermodynamic Parameters of Transition Metal Oxides, in: Proc, 4th Internat. Congr. Catalysis (Moscow, 1968), Amsterdam, Elsevier, 1969, p. 454.

72. W.M.H. Sachtler, G.J.H. Dorgelo, J. Fahrenfort, and R.J.H. Voorhoeve, Rec. Trav. Chim. Pays Bas 89 (1970) 460.

73. Yu.D. Pankratiev, React. Kinet. Catal. Lett. 20 (1982) 255.

74. W.van Gool and A.G. Piken, J. Mater. Science 4 (1969), 95, 105.

75. H.H. Kung, J. Catal. 73 (1982) 387.

76. H.H. Kung and M.C. Kung, Selective Oxidative Dehydrogenation of Butenes on Ferrite Catalysts, in: Adv. Catalysis, Vol. 33 (1985) 159.

77. M.P.van Dijk, A.J. Burgeraaf, A.N. Cormack and C.R.A. Catlow, Solid State Ionics, 17 (1985) 159.

78. M.P.van Dijk, F.C. Mijlhof and A.J. Burggraaf, J. Solid State Chem. 62 (1985) 377.

79. M.P.van Dijk, KJ. de Vries and A.J. Burggraaf, Solid State Ionics 16 (1985) 211.

80. J.C. Boivin and J.D. Thomas, Solid State Ionies 5 (1981) 523. 
81. P. Conflant, J.C. Boivin, G. Nowogrocki, and D.J. Thomas, Solid State Ionics $9 / 10$ (1983) 925.

82. M.P.van Dijk, KJ, de Vries and AJ. Burggraaf, Solid State Ionics 9/10 (1983) 913.

83. Z, Wuzong, D.A. Jefferson, M. Alario-Franco and J.M. Thomas, J. Phys. Chem. 91 (1987) 512.

84. T. Kudo and H. Obayashi, J. Electrochem. Soc. 122 (1975) 142.

85. R.L. Cook, R.C. MacDuff, and A.F. Sammells, J. Electrochem. Soc. 137 (1990) 3309.

86. R.L. Cook and A.F. Sammells, Solid State Ionics 45 (1991) 311.

87. H.A. Harwig and A.G. Gerards, J. Solid State Chem. 26 (1978) 265.

88. J.B. Torrance, Ph. Lacorro, Ch. Asavaroengchai, and R.M. Metzger, J. Solid State Chem. 90 (1991) 168.

89. H. Iwahara, T. Esaka, and Y. Miyawaki, Solid State lonics 44 (1991) 257.

90. J. Mizusaki, Y. Mima. S. Yamauchi, K. Fueki, and H. Tagawa, J. Solid State Chem. 80 (1989) 102.

91. A. Belzner, T.M. Gür and R.A. Huggins, Measurement of the Chemical Diffusion Coefficient of Oxygen in $\left(\mathrm{Lat}_{0.7} \mathrm{Sr}_{0.2}\right) \mathrm{MnO}_{3-8}$, in: Proc. 1st Int. Symposium on Solid Oxide Fuel Cells, ed. S.C. Singhal, Pennington NJ, The Electrochemical Society, Vol. 89-11 (1989) 214.

92. T. Ishigaki, S. Yamauchi, K. Kishio, J. Mizusaki, and K. Fueki, J. Solid State Chem. 73 (1988) 179.

93. Y. Oishi, K. Ando and M. Akiyama, Nippon Kagaku Kaishi 1981, 1445.

94. R.N. Blumenthal, F.S. Bruger and J.E. Garnier, J. Electrochem. Soc. 120 (1973) 1230.

95. I. Ishikagi, S. Yamauchi, J. Misuzaki, K. Fueki, and H. Tamaru, J. Solid State Chem. 54 (1984) 100.

96. I. Ishikagi, S. Yamauchi, J. Misuzaki, H. Naito, and T. Adachi, J. Solid State Chem. 55 (1984) 50.

97. M.A. Priestnall and B.C.H. Steele, Strontium-Doped Lanthanum Copper Oxide as a Solid Oxide Fuel Cell Cathode Material, in: Proc. 1st Int. Symposium on Solid Oxide Fuel Cells, ed. S.C. Singhal, Pennington NJ, The Electrochemical Society, Vol. 89-11 (1989) 157.

98. L. Bates, WJ. Weber and C.W. Griffing, Solid Oxide Fuel Cell Electrodes Based on $\mathrm{In}_{2} \mathrm{O}_{3}-\mathrm{PrO}_{183}-\mathrm{ZrO}_{2}$, in: Proc. 1st Int. Symposium on Solid Oxide Fuel Cells, ed. S.C. Singhal, Pennington NJ, The Electrochemical Society, Vol. 89-11 (1989) 141.

99. A. Hammouche, EJ.L. Schouler, and M. Henault, Solid State Ionics 28-30 (1988) 1205.

100. A. Hammouche, E. Siebert, and A. Hammou, Mat. Res. Bull. 24 (1989) 367.

101. A. Hammouche, E. Siebert, M. Kleitz, and A.L. Hammou, Oxygen Reduction at the $\mathrm{La}_{1-\mathrm{x}} \mathrm{Sr}_{\mathrm{x}} \mathrm{MnO}_{3} /$ Zirconia Electrode, in: Proc. Ist Int. Symposium on Solid Oxide Fuel Cells, ed. S.C. Singhal, Pennington NJ, The Electrochemical Society, Vol. 89-11 (1989) 265.

102. Y. Takasu, T. Sugino and Y. Matsuda, J. Appl. Electrochem. 14 (1984) 79.

103. M.P.van Dijk, Ordering, electrical conductivity and electrode properties of materials with fluorite related structures, Ph.D. Thesis, University of Twente, 1985.

104. B.A. Boukamp, M.P. van Dijk, KJ. de Vries and A.J. Burggraaf, Electrochemical Properties of Nonstoichiometric Solid Oxides, in: Nonstoichiometric Compounds, eds. C.R. Catlow and W.Mackrodt, Adv. in Ceramics, Vol. 23 (1987) p. 447.

105. S.S. Liou and W.L. Worrell, Mixed-Conducting Oxide Electrodes for Solid Oxide Fuel Cells, in: Proc. 1st Int. Symposium on Solid Oxide Fuel Cells, ed. S.C. Singhal, Pennington NJ, The Electrochemical Society, Vol. 89-11 (1989) 81.

106. L. Boon and R. Metselaar, Mixed Conductivity in Catalytic Active Bismuth Molybdates, in: Proc. of the 10th Int. Conf. on the Reactivity of Solids, Dijon, France, aug. 1984, eds. P. Barret and L.-C.Dufour, Amsterdam, Elsevier, 1985, p. 357.

107. L. Boon and R. Metselaar, Solid State Ionies 16 (1985) 201.

108. L. Boon and R. Metselaar, Eur. J. Solid State Inorg. Chem. 27 (1990) 381.

109. S.E. Golunski and Th.G. Nevell, J. Chem. Soc. Faraday Trans. 1, 81 (1985) 1121.

110. R.K. Grasselli and D.D. Suresh, J. Catal. 14 (1969) 93.

111. B.C. Gates, J.R. Katzer and G.C.A. Schuit, Chemistry of Catalytic Processes, New York, MacGrawHill, 1979 , ch. 4. 
112. A. Ovenston, J.R. Walls and S. Miri, Solid State Ionies, 28-30 (1988) 1553

113. A. Ovenston, J.R. Walls, S. Miri, and T. Ramdeen, J. Phys. D: Appl. Phys. 21 (1988) 1773.

114. A.M. Stoneham and P.W. Tasker, The Theory of Ceramic Surfaces, in: Surface and Near Surface Chemistry of Oxide Materials, eds. L.-C. Dufour and J. Nowotny, Amsterdam, Elsevier, 1988, p. 1.

115. P.W. Tasker, Surf. Sci. 78 (1979) 315.

116. A.J. Burggraaf and A.J.A. Winnubst, Segregation in Oxide Surfaces, Solid Electrolytes and Mixed Conductors, in: Surface and Near Surface Chemistry of Oxide Materials, eds. L.-C. Dufour and J. Nowotny, Amsterdam, Elsevier, 1988, p. 449.

117. A.J. Burggraaf, M. van Hemert, D. Scholten and A.J.A. Winnubst, in: Proc. of the 10th Int. Conf. on the Reactivity of Solids, Dijon, France, aug. 1984, eds. P. Barret and L.-C.Dufour, Amsterdam, Elsevier, 1985, p. 797.

118. J. Nowotny, Solid State Ionics 28-30 (1988) 1235.

119. P. Wynblatt and R.C. McCune, Surface Segregation in Metal Oxides, in: Surface and Near Surface Chemistry of Oxide Materials, eds. L.-C. Dufour and J. Nowotny, Amsterdam, Elsevier, 1988, 247.

120. R.G. Egdell and W.C. Mackrodt, Theory of Dopant Segregation in Ceramic Oxides, in: Surfaces and Interfaces of Ceramic Materials, eds. L.-C. Dufour, C. Monty and G. Petot-Ervas, Dordrecht, Kluwer Academic Press, 1989, p. 185.

121. J. Nowotny and J.B. Wagner, Jr., Oxid. Metals 15 (1981) 169.

122. Z. Adamczyk and J. Nowotny, J. Phys. Chem. Solids 47 (1986) 11.

123. A.J.A. Winnubst, P.J.M. Kroot and A.J. Burggraaf, J. Phys. Chem. Solids. 44 (1983) 955.

124. A.J. Burggraaf, M. van Hemert, D. Scholten and A.J.A. Winnubst, in: Proc. of the 10th Int. Conf. on the Reactivity of Solids, Dijon, France, aug. 1984, eds. P. Barret and L.-C.Dufour, Amsterdam, Elsevier, 1985, p. 797.

125. G.S.A.M. Theunissen, A.J.A. Winnubst and A.J. Burggraaf, Segregation in $\mathrm{ZrO}_{2}-\mathrm{Y}_{2} \mathrm{O}_{3}$ Ceramics, in: Surfaces and Interfaces of Ceramic Materials, eds. L.-C. Dufour, C. Monty and G. Petot-Ervas, Dordrecht, Kluwer Academic Press, 1989, p. 365.

126. J. Nowotny, M. Sloma and W. Weppner, Solid State Ionies, 32/33 (1989) 709.

127. F. Figueras, M. Forissier, J.P. Lacharme and J.L. Portefaix, Applied Catal. 19 (1985) 21.

128. A.J. Burggraaf, P.J. Gellings and D. Scholten, Ion implantation and (mono)layer techniques for modifying ceramic surfaces., in: High Tech Ceramics, ed. by P. Vincenzini, Amsterdam, Elsevier, 1987, p. 779 - 794.

129. A.J. Burggraaf, K. Keizer and B.A. van Hassel, Nanophase Ceramics, Membranes and Ion Implanted Layers, in: Surfaces and Interfaces of Ceramic Materials, eds. L.-C. Dufour, C. Monty and G. Petot-Ervas, Dordrecht, Kluwer Academic Press, 1989, p. 705.

130. B.A.van Hassel and A.J. Burggraaf, Appl. Phys. A 49 (1989) 33.

131. S.R. Morrison, The Chemical Physics of Surfaces, Plenum, New York (1977).

132. J. Nowotny and M. Sloma, Work Function of Oxide Ceramic Materials, in: Surface and Near Surface Chemistry of Oxide Materials, eds. L.-C. Dufour and J. Nowotny, Amsterdam, Elsevier, 1988, p. 281.

133. J. Maier, Solid State Ionics, 32/33 (1989) 727.

134. J. Maier, Ber. Bunsenges. Phys. Chem. 88 (1984) 1057.

135. J. Maier, J. Phys. Chem. Solids 46 (1985) 309.

136. J. Maier, Ber. Bunsensges. Phys. Chem. 89 (1985) 355.

137. J. Maier, J. Electrochem. Soc. 134 (1987) 1524.

138. W. Göpel, Charge Transfer Reactions on Semiconductor Surfaces, in: Festkörperprobleme, XX (1980) 177.

139. W. Göpel, Sensors and Actuators, 16 (1989) 167.

140. A. Bielanski and J. Haber, Oxygen in Catalysis, New York, Marcel Dekker, Inc., 1991. 
141. M. Che and A.J. Tench, Characterization and Reactivity of Mononuclear Oxygen Species on Oxide Surfaces, in: Advances in Catalysis, Academic Press, New York, 1982, vol. 31, p. 77.

142. M. Che and A.J. Tench, Characterization and Reactivity of Molecular Oxygen Species on Oxide Surfaces, in: Advances in Catalysis, Academic Press, New York, 1983, vol. 32, p. 1.

143. J. Lamotte, J.-C. Lavalley, V. Lorenzelli, and E. Freund, J. Chem. Soc., Faraday Trans.1, 81 (1985) 215.

144. M. Che, K. Dyrek, M. Kermarec, and A.J. Tench, Rev. Chim. Miner. 21 (1984) 669.

145. Y. Takasu, M. Matsui, H. Tamura, S. Kawamura, Y. Matsuda and I. Toyoshima, J. Catal. 69 (1981) 51.

146. Y. Takasu, M. Matsui and Y. Matsuda, J. Catal. 76 (1982) 61.

147. Y. Takasu, T. Yoko-o, M. Matsui, Y. Matsuda and I. Toyoshima, J. Catal. 77 (1982) 485.

148. K. Otsuka and T. Nakajima, J. Catal. 102 (1987) 216.

149. H.M. Zhang, Y. Shimizu, Y. Teraoka, N. Miura, and N. Yamazoe, J. Catal. 121 (1990) 432.

150. N. Yamazoe, S. Furukawa, Y. Teraoka, and T. Seiyama, Chem. Lett. 19822019.

151. Y. Teraoka, M. Yoshimatsu, N. Yamazoe, and T. Seiyama, Chem. Lett. 1984893.

152. P.E. Childs and J.B. Wagner, Jr., Heterogeneous Kinetics at Elevated Temperature, Plenum Press, New York, 1970, 9. 269.

153. P.E. Childs, L.W. Laub and J.B. Wagner, Jr., Proc. Br. Ceram. Soc. 19 (1973) 29.

154. F. Morin, Can. Metall. O. 14 (1975) 97.

155. F. Gesmundo, Solid State Ionics 12 (1984) 79.

156. A.S. Bayoglu and R. Lorenzelli, Solid Sate Ionics 12 (1984) 53.

157. W.T. Petusky and H.K. Bowen, J. Am. Ceram. Soc. 64 (1981) 611.

158. P. Guedeney, R. Ducroux and A. Pattoret, Solid State Ionics 12 (1984) 161.

159. L. Heyne and N.M. Beekmans, Proc. Brit. Ceram. Soc. 19 (1971) 229.

160. S. Dou, C.R. Masson and P.D. Pacey, J. Am. Ceram. Soc. 72 (1989) 1114.

161. G.J. Koel and P.J. Gellings, Oxid. Met. 5 (1972) 185.

162. J.B. Price and J.B. Wagner, Jr., Z. Phys. Chem. 49 (1966) 257.

163. J. Crank, The Mathematics of Diffusion, 2nd ed, Oxford University Press, London/New York, 1975, p. 94.

164. H.J. Grabke, Ber. Bunsenges. Physik. Chem. 69 (1965) 49.

165. L.W. Laub and J.B. Wagner, Jr., Oxid. Met. 7 (1973) 1.

166. I. Bransky, N.M. Tallan, J.M. Wimmer and M. Gwishi, J. Am. Ceram. Soc. 54 (1971) 26.

167. A.B. Neuman, Trans. Am. Inst. Chem. Eng. 27 (1931) 203.

168. F. Viani, V. Doni and F. Gesmundo, Oxid. Met. 21 (1984) 309.

169. V. Dovi, F. Gesmundo and F. Viani, Oxid. Met. 23 (1985) 35.

170. F. Gesmundo, F. Viani and V. Dovi, Oxid. Met. 23 (1985) 141.

171. S. Dou, C.R. Masson and P.D. Pacey, J. Electrochem. Soc. 132 (1985) 1843.

172. H.J.M. Bouwmeester, H. Kruidhof, P.J. Gellings and A.J. Burggraaf, Proceedings Third International Symposium on Systems with Fast Ionic Transport, Holzhau/Erzgebirge, Germany, April 1991, in press.

173. J. Nowotny and J.B. Wagner, Jr., Oxid. Met. 15 (1981) 169.

174. Z. Adamczyk and J. Nowotny, J. Phys. Chem. Solids, 47 (1986) 11.

175. J. Nowotny, Solid State Ionics, 28-30 (1988) 1235.

176. Z. Adamczyk and J. Nowotny, Diffusion and Defect Data, Solid Sate Data-Pt.B, Vol. 15 \& 16, Interface Segregation and Related Properties in Materials, ed. J. Nowotny (1991) p. 285.

177. K. Weiss, Z. Phys. Chem. N.F. 59 (1968) 242.

178. K. Weiss, Ber. Bunsenges. Phys. Chem. 73 (1969) 344. 
179. D.O. Raleigh, Progr. Solid State Chem., Vol. 3, Pergamon Press, Oxford, 1967, p. 83.

180. J.B. Wagner, Jr., Electrode Processes, in: Solid State lonies, eds. M. Kleitz and J. Dupuy, D. Reidel Publishing Company, Dordrecht-Holland, 1976, p. 185.

181. W. Weppner and R.A. Huggins, Ann. Rev. Mater. Sci. 8 (1978) 269.

182. L.D. Burke, H. Rickert and R. Steiner, Z. Phys. Chem. N.F. 74 (1971) 146.

183. W. Weppner, Z. Naturforsch. 31a (1976) 1336.

184. W. Weppner, Electrochim. Acta 22 (1977) 721.

185. H. Rickert and W. Weppner, Z. Naturforsch. 29a (1974) 1849.

186. A. Belzner, T.M. Gür and R.A. Huggins, Solid State Ionics 40/41 (1990) 535.

187. G. Heiland and D. Kohl, Chemical Sensor Technology, ed. T. Seyama, Elsevier, Amsterdam, Vol. 1, 1988, p. 15.

188. T. Kudo, Catal. Today 8 (1990) 263.

189. K. Takahata, Chemical Sensor Technology, ed. T. Seyama, Elsevier, Amsterdam, Vol. 1, 1988, p.39.

190. Y. Oishi and W.D. Kingery, J. Chem. Phys. 33 (1960) 480.

191. K. Ando, Y. Oishi, H. Koizama and Y. Sakka, J. Mat. Sc. Lett. 4 (1985) 176.

192. K. Park and D.R. Olander, J. Electrochem. Soc. 138 (1991) 1154.

193. J.A. Kilner, B.C.H. Steele and L. Ilkov, Solid State Ionics 12 (1984) 89.

194. R.J. Tarento and C. Monty, Solid State Ionics 28-30 (1988) 1221.

195. S. Yamauchi, T. Ishigaki, J. Misuzaki and K. Fueki, Solid State Ionics 9 \& 10 (1983) 997.

196. T. Ishigaki, S. Yamauchi, K. Kishio, J. Mizusaki and K. Fueki, J. Solid State Chem. 73 (1988) 179.

197. L.A. Simpson and R.E. Carter, J. Am. Ceram. Soc. 49 (1966) 140.

198. G. Parravano, Catal. Rev. 3 (1969) 207.

199. G.I. Golodets, "Heterogeous Catalytic Reactions Involving Molecular Oxygen", in: "Studies in Surface Science and Catalysis", Elsevier, New York, Vol. 15, 1983, p.181.

200. G.K. Boreskov, Adv. Catal. 15 (1964) 285.

201. G.K. Boreskov, Discuss. Farad. Soc. 41 (1966) 263.

202. E.R.S. Winter, J. Chem. Soc. (A) (1968) 479.

203. J. Novakova, Catal. Rev. 4 (1970) 77.

204. G.K. Boreskov and V.S. Muzykantov, Ann. New York, Acad. Sci. 213 (1973) 137.

205. G.K. Boreskov in: "Catalysis, Science and Technology", Springer-Verlag, New York, Vol. 3, 1982, p. 39 .

206. B.A. Boukamp, I.C. Vinke, K.J. de Vries and A.J. Burggraaf, Solid State Ionics 32/33 (1989) 918.

207. V.V. Popovkkii and G.K. Boreskov, Kinet. Catal. 1 (1960) 566.

208. K. Klier, J. Novakova and P. Jiru, J. Catal. 2 (1963) 479.

209. V.S. Muzykantov, V.V. Popovskii and G. Boreskov, Kinet. Catal. 5 (1964) 624.

210. H.A.C. McKay, Nature 142 (1938) 997.

211. T. Takaishi, Bull. Chem. Soc. Japan 42 (1969) 1266.

212. K.F. Bonhoeffer and A. Farkas, Z. Phys. Chem. B12 (1931) 231.

213. M.P. Rosynek, Catal. Rev. Sci. Eng. 16 (1977) 111.

214. G.V. Antoshin, Kh. M. Minachev and R.V. Dmitriev, Kinet. Katal. 9 (1967) 1968.

215. E.R.S. Winter, J. Chem. Soc. (1969) 1832.

216. K. Klier and E. Kucera, J. Phys. Chem. Solids 27 (1966) 1087.

217. V.S. Muzykantov and G.I. Panov, Kinet. Katal. 13 (1972) 350.

218. A.K. Avetisov, N.D. Gol'dstein, D.A. Dulin, A. Mischenko and A.I. Gei'bshtein, J. Catal. 52 (1978) 1.

219. V.S. Muzykantov, G.I. Panov and G.K. Boreskov, Kinet. Katal. 14 (1973) 948. 
220. F.J. Barry and F.S. Stone, Proc. Roy. Soc., London, Ser. A255 (1960) 124.

221. J. Novakova, K. Klier and P. Jiru, Reactivity of Solids 5th International Symposium, Munich, Elsevier, Amsterdam, 1964, p. 1965.

222. B.A. Boukamp, K.J. de Vries and A.J. Burggraaf, Surface Oxygen Exchange in Bismuth Oxide Based Materials, in: Non-Stoichiometric Compounds, Surfaces, Grain Boundaries and Structural Defects, eds. J. Nowotny and W. Weppner, Dordrecht, Kluwer, 1989, p. 299.

223. I.C. Vinke, Electrochemical and Electrode Properties of Bismuth Oxide Ceramics, Thesis, University of Twente, the Netherlands, 1991.

224. H.J.M. Bouwmeester, B.A. Boukamp, B. Löscher, P.J. Gellings and A.J. Burggraaf, to be published.

225. C. Li, K. Domen, K.I. Maruya and T. Onishi, J. Catal. 123 (1990) 436.

226. S.C. Chang, J. Vac. Sci. Technol. 17 (1980) 366.

227. H.L. Tuller, J.A. Kilner, A.E. McHale and B.C.H. Steele, Proc. 10th Reactivity of Solids (1985) p. 315.

228. B.C.H. Steele, J.A. Kilner, P.F. Dennis, A.E. McHale, M. van Hemert and A.J. Burggraaf, Solid State Ionics 18 \& 19 (1986) 1038.

229. X. Turrillas, J.A. Kilner, I. Kontoulis and B.C.H. Steele, J. Less-Common. Met. 151 (1989) 229.

230. E.Kh. Kurumchin and M.V. Perfil'ev, Solid State Ionics 42 (1990) 129.

231. V.P. Ishchuk, E. Kh. Kurumchin, M.V. Perfil'ev and S.V. Karpachev, Kinet. Katal. 21 (1979) 714.

232. G.W. Keulks, J. Catal. 19 (1970) 232.

233. J. Novakova and P. Jiru, J. Catal. 27 (1972) 155.

234. C.J. Wen, D.M. Mason, J. Appl. Electrochem. 8 (1978) 81.

235. D. Yuan and F.A. Kröger, J. Electrochem. Soc. 116 (1969) 594.

236. H. Yanagida, R.J. Brook and F.A. Kröger, J. Electrochem. Soc. 117 (1970) 593.

237. E.J.L. Schoulcr and M. Kleitz, J. Elcctrochcm. Soc. 134 (1987) 1045.

238. B. Van Hassel, Thesis, University of Twente, The Netherlands (1990).

239. T. Inou, K. Eguchi and H. Arai, Chem. Lett. (1988) 1939.

240. T. Inou, N. Seki, K. Eguchi and H. Arai, J. Electrochem. Soc. 137 (1990) 2523.

241. M.P. van Dijk, K.J. de Vries and A.J. Burggraaf, Solid State Ionics 21 (1986) 73.

242. B.A. Boukamp, H.J.M. Bouwmeester, A.J. Burggraaf and P.J. Gellings, to be published.

243. Y. Takeda, R. Kanno, M. Noda, Y. Tomida, and O. Yamamoto, J. Electrochem. Soc. 134 (1987) 2656.

244. V.V. Popovskii, G.K. Boreskov, V.S. Muzykantov, V.A. Sazonov and S.G. Shubnikov, Kinet. Katal. 10 (1969) 786.

245. V.V. Popovskii, G.K. Boreskov, Z. Dzeventski, V.S. Muzykantov and T.T. Shul'meister, Kinet. Katal. 12 (1970) 979.

246. V.S. Muzykantov, V.V. Popovskii, G.K. Boreskov, G.I. Panov and R.A. Shrabina, Kinet. Katal. 13 (1972) 385.

247. G.K. Boreskov, V.V. Popovskii, N.I. Lebedeva, V.A. Sazonv and T.V. Adrushkevich, Kinet. Katal. 5 (1969) 1253.

248. J. Haber, Kinet. Katal. 21 (1980) 123.

249. S. Dou, C.R. Masson and P.D. Pacey, Solid State Ionics, $18 \& 19$ (1986) 736.

250. Y. Teraoka, H.-M. Zhang, S. Furukawa, and N. Yamazoe, Chem. Letters, 1985, 1743.

251. Y. Teraoka, T. Nobunaga, and N. Yamazoe, Chem. Letters, 1988, 503.

252. A.J. Burggraaf, H.J.M. Bouwmeester, B.A. Boukamp, R.J.R. Uhlhorn, and V. Zaspalis, Synthesis, Microstructure and Properties of Porous Ceramic Membranes, in: The Science of Ceramic Interfaces, ed J. Nowotny, Elsevier Sc. Publ. 1991, in press.

253. W.C. Maskell and B.C.H. Steele, J. Appl. Electrochem. 59 (1986) 1997. 
254. J. Fouletier, P. Fabry and M. Kleitz, J. Electrochem. Soc. 123 (1976) 204.

255. M. Kleitz and E. Siebert, Electrode Reactions in Potentiometric Gas Sensor, in: Chemical Sensor Technology, Vol. 2, ed. T. Seyama, Elsevier Science publishers B.V., Amsterdam, 1989, p. 151.

256. Y. Nigara and B. Cales, Bull. Chem. Soc. Japan 59 (1986) 1997.

257. B. Cales and J.F. Baumard, High Temp.-High Pressures 14 (1982) 681.

258. R. Di Cosimo, J.D. Burrington, and R.K. Grasselli, J. Catal. 102 (1986) 234.

259. J.H.H.ter Maat, M.P. van Dijk, G. Roclofs, H. Bosch, G.M.H. van de Velde, P.J. Gellings, and A.J. Burggraaf, Mat. Res. Bull. 19 (1984) 1271.

260. E.R.S. Winter, J. Catal. 15 (1969) 144.

261. E.R.S. Winter, J. Catal. 19 (1970) 32.

262. E.R.S. Winter, J. Catal. 34 (1974) 431.

263. W. Ueda and J.M. Thomas, J. Chem. Soc., Chem. Comm. 1988, 1148.

264. A.B. Anderson, Y. Kim, D.W. Ewing, R.K. Grassclli and M. Tenhover, Surf. Science 134 (1983) 237.

265. N. Yamazoe and Y. Teraoka, Catalysis Today 8 (1990) 175.

266. N. Bonanos, B. Ellis, K.S. Knight, and M.N. Mahmood, Solid State Ionics 35 (1989) 179.

267. F. Abraham, J.C. Boivin, G. Mairesse, and G. Nowogrocki, Solid State Ionics 40/41 (1990) 934.

268. R.J.H. Voorhoeve, D.W. Johnson, Jr., J.P. Remeika, and P.K. Gallagher, Science 195 (1977) 827.

269. N. Mizuno, Catalysis Today, 8 (1990) 221.

270. J.L. Callahan and R.K. Grasselli, A.I.Ch.E. Journal 9 (1963) 755.

271. P. Mars and D.W. van Krevelen, Chem. Eng. Sci. Suppl., 3 (1954) 41.

272. C.N. Satterfield, Heterogeneous Catalysis in Practice, New York, McGrawHill, 1980, ch. 8.

273. R.J.H. Voorhoeve, J.P. Remeika and L.E. Trimble, Ann. N.Y. Acad. Sci. 272 (1976) 3.

274. M. Breysse, M. Guenin, B. Claudel, H. Latrielle and J. Veron, J. Catal. 27 (1972) 275.

275. M. Breysse, M. Guenin, B. Claudel, and J. Veron, J. Catal. 28 (1972) 54.

276. G.W. Berkstresser, R.J. Brook, and J.M. Whelan, J. Mater. Sci. 9 (1974) 491.

277. M.P.van Dijk, J.H.H. ter Maat, G. Roelofs, H. Bosch, G.M.H. van de Velde, P.J. Gellings, and A.J. Burggraaf, Rec. Trav. Chim. Pays Bas 103 (1984) 38.

278. M.P.van Dijk, J.H.H. ter Maat, G. Roelofs, H. Bosch, G.M.H. van de Velde, P.J. Gellings, and A.J. Burggraaf, Mat. Res. Bull. 19 (1984) 1149.

279. M.J. Verkerk and A.J. Burggraaf, J. Electrochem. Soc. 130 (1983) 70.

280. S.J. Korf, H.J.A. Koopmans, B.C. Lippens, Jr., A.J. Burggraaf, and P.J. Gellings, J. Chem. Soc. Faraday Trans.1, 83 (1987) 1485.

281. A. Andersson, J. Catal. 69 (1981) 465, 70 (1982) 144.

282. P. Courtine, Thermodynamic and Structural Aspects of Interfacial Effects in Mild Oxidation Catalysts, in: Solid State Chemistry in Catalysis, eds. R.K. Grasselli and J.F. Brazdil, ACS Symposium Series 279, Washington D.C., American Chemical Society, 1985, p. 37.

283. K. Otsuka and M. Kunitomi, J. Catal., 105 (1987) 525.

284. D.Y. Rao and D.K. Chakrabarty, Ind. J. Chem. 23A (1984) 375.

285. J.M.D. Tascon, J.L. Garcia Fierro, and L.G. Tejuca, Z. physik. Chem. N.F. 124 (1981) 249.

286. K. Tabata, I. Matsumoto, and S. Kohiki, Catalytic Properties and Surface States of Perovskite-type Mixed Oxides, in: Proc. 9th Int. Congr. Catalysis, Calgary, 1988, Ottawa, The Chemical Institute of Canada, vol. 4 (1988) p. 1812.

287. T. Nakamura, M. Misono, and Y. Yoneda, Chem. Lett. 19811589.

288. T. Nakamura, M. Misono, and Y. Yoneda, Bull. Chem. Soc. Jpn. 55 (1982) 394.

289. P.K. Gallagher, D.W. Johnson, Jr., and F. Schrey, Mat. Res. Bull. 9 (1974) 1345.

290. W.F. Chu and F.J. Rohr, Solid State Ionics, 28-30 (1988) 1540.

291. M.W. Chien, I.M. Pearson, and K. Nobe, Ind. Eng. Chem., Prod. Res. Dev., 14 (1975) 131. 
292. N. Mizuno, M. Yamato, and M. Misono, J. Chem. Soc., Chem. Commun., 1988887.

293. K. Tabata, H. Fukuda, S. Kohili, N. Mizuno, and M. Misono, Chem. Lett. 1988799.

294. T. Arakawa and G. Adachi, Mat. Res. Bull. 24 (1989) 529.

295. P. Pomonis and J.C. Vickerman, J. Catal. 90 (1984) 305.

296. S. Shin, H. Arakawa, Y. Hatakeyama, K. Ogawa, and K. Shimomura, Mat. Res. Bull. 14 (1979) 633.

297. Y. Teraoka, H. Fukuda, and S. Kagawa, Chem. Lett. 1990, 1.

298. D.B. Dadyburjor and E. Ruckenstein, J. Phys. Chem. 82 (1978) 1563.

299. J.D. Burrington and R.K. Grasselli, J. Catal. 59 (1979) 79.

300. L.D. Krenzke and G.W. Keulks, J. Catal. 64 (1980) 295.

301. H. Miura, Y. Arai, K. Sugiyama and T. Matsuda, J. Catal. 68 (1981) 264.

302. J.R. Monnier and G.W. Keulks, J. Catal. 68 (1981) 51.

303. J.F. Brazdil, L.C. Glaeser, and R.K. Grasselli, J. Catal. 81 (1983) 142.

304. R.K. Grasselli and J.D. Burrington, Selective Oxidation and Ammoxidation of Propylene by Heterogeneous Catalysts, in: Adv. in Catalysis, eds. D.D. Eley, H. Pines, and P.B. Weisz, New York, Academic Press, 30 (1981) 133.

305. R.K. Grasselli, Appl. Catal. 15 (1985) 127.

306. T.P. Snyder and C.G. Hill, Jr., Catal. Rev.-Sci. Eng. 31 (1989) 43.

307. R.K. Grasselli, J.F. Brazdil, and J.D. Burrington, Solid State and Surface Mechanisms in Selective Oxidation and Ammoxidation Catalysts, in: Proc. 8th Int. Congr. Catal., Weinheim, Verlag Chemie, 1984, vol. V., p. 369.

308. L.C. Glaeser, J.F. Brazdil, M.A. Hazle, M. Mehicic and R.K. Grasselli, J. Chem. Soc. Faraday Trans. 1,81 (1985) 2903.

309. R.K. Grasselli, React. Kinet. Catal. Lett., 35 (1987) 327.

310. P.M. Burban, G.C.A. Schuit, T.A. Koch, and K.B. Bischoff, J. Catal. 126 (1990) 317.

311. P.M. Burban, G.C.A. Schuit, T.A. Koch, and K.B. Bischoff, J. Catal. 126 (1990) 326.

312. N. Hykaway, W.M. Sears, R.F. Frindt and S.R. Morrison, Sensors and Actuators 15 (1988) 105.

313. W.M. Sears, Sensors and Actuators, 19 (1989) 351.

314. W.M. Sears, Bulletin of the Bismuth Inst. 62 (1991) 1.

315. F. Theobald, A. Laarif, and A.W. Hewat, Mat. Res. Bull. 20 (1985) 653.

316. T. Hayakawa, T. Tsunoda, H. Orita, T. Kameyama, H. Takahashi, K. Fukuda, and K. Takehira, J. Chem. Soc., Chem. Comm., 1987, 780.

317. R.K. Grasselli, J.D. Burrington, and J.F. Brazdil, Faraday Discuss. Chem. Soc. 72 (1982) 203.

318. W. Ueda, K. Asakawa, C-1. Chen, Y. Moro-oka, and T. Ikawa, J. Catal. 101 (1986) 360.

319. T. Hayakawa, T. Tsunoda, H. Orita, T. Kameyama, M. Ueda, K. Fukuda, and K. Takehira, J. Chem. Soc., Chem. Comm., 1988, 1593.

320. K.M. Sancier, P.R. Wentrcek and H. Wise, J. Catal. 39 (1975) 141.

321. G.W. Keulks, J. Catal. 19 (1970) 232.

322. G.W. Keulks and L.D. Krenzke, The Role of Lattice Oxygen in Selective and Complete Oxidation of Propylene over Binary Oxide Catalysts, in: Proc. 6th Int. Congr. Catal. London, Vol. B, 1976, p. 806.

323. G. Centi and F. Trifirò, Catal. Rev. - Sci. Eng. 28 (1986) 165.

324. D.A.G. van Oeffelen, J.H.C. van Hooff, and G.C.A. Schuit, J. Catal. 95 (1985) 84.

325. J. Roos, Catalytic Activation of Methane, Thesis University of Twente, 1989.

326. Y. Amenomiya, V.I. Birss, M. Goledzinowski, J. Galuszka, and A.R. Sanger, Catal. Rev. - Sci. Eng. 32 (1990) 163.

327. K. Otsuka, K. Jinno, and A. Morikawa, Chem. Lett. 1985499.

328. K. Otsuka, K. Jinno, and A. Morikawa, J. Catal. 100 (1986) 353. 
329. V.B. Balakireva, A.D. Neumin, and S.F. Pal'guev, Dokl. Akad. Nauk SSSR 261 (1981) 121, cited from Chem. Abstr. 96 (1982) $78230 \mathrm{~b}$.

330. J.M. Deboy and R.F. Hicks, J. Catal. 113 (1988) 517.

331. J.M. Thomas, W. Ueda, J. Williams, and K.D.M. Harris, Faraday Discuss. Chem. Soc. 87 (1989) 33.

332. K. Machida and M. Enyo, J. Chem. Soc., Chem. Commun. 1987, 1639.

333. L.S. Woldman and V.D. Sokolovskii, Catal. Lett. 8 (1991) 61.

334. P-H. Chiang, D. Eng and M. Stoukides, J. Electrochem. Soc. 138 (1991) L11.

335. H. Arai, T. Yamada, K. Eguchi, and T. Seiyama, Appl. Catal. 26(1986) 265.

336. G. Kremenit, J.M.L. Nieto, J.M.D. Tascón, and L.G. Tejuca, J. Chem. Soc., Faraday Trans. 1, 81 (1985) 939.

337. J.G. McCarty, M.A. Quinlan, and H. Wise, Catalytic Combustion of Methane by Complex Oxides, in: Proc. 9th Int. Congr. Catalysis, Calgary, 1988, Ottawa, The Chemical Institute of Canada, vol. 4 (1988) p. 1818.

338. B.C.H. Steele, I. Kelly, H. Middleton and R. Rudkin, Solid State Ionies, 28-30 (1988) 1547.

339. M. Mogensen and J.J. Bentzen, Oxidation of Methane on Oxide Electrodes at 800 to $1000^{\circ} \mathrm{C}$, in: Proc. 1st Int. Symposium on Solid Oxide Fuel Cells, ed. S.C. Singhal, Pennington NJ, The Electrochemical Society, Vol. 89-11 (1989) 99.

340. T.M. Gür, H. Wise and R.A. Huggins, J. Catal. 129 (1991) 216.

341. M.T. Mirza, J.R. Walls, and S.A.A. Jayaweera, Thermochim. Acta. 152 (1989) 203.

342. N. Tsuda, T. Mori, and Y. Sakai, J. Mol. Catal. 28 (1985) 183.

343. N. Tsuda and A. Fujimori, J. Catal. 69 (1981) 410.

344. N. Kosaka, Y. Sakai, and N. Tsuda, J. Catal, 98 (1986) 95.

345. J.O. Petunchi and E.A. Lombardo, Catalysis Today 8 (1990) 201.

346. R.G. Silver, C.J. Hou and J.G. Ekerdt, J. Catal. 118 (1989) 400.

347. N.B. Jackson and J.G. Ekerdt, J. Catal. 101 (1986) 90.

348. N.B. Jackson, and J.G. Ekerdt, J. Catal. 126 (1990) 46.

349. F. Trifiro and A. Vaccari, in: Symposium on Structure-Activating Relationships in Heterogeneous Catalysis, Boston, American Chemical Society, 1990, p.83.

350. R.A. Jackson, C.R.A. Catlow, and J.M. Thomas, Catal. Lett. 8 (1991) 385.

351. M.A. Ryashentseva, A.A. Dulov, L.A. Abramova, O.P. Tkachenko, V.V. Fomichev, and A.E. Vetrov, J. Catal. 125 (1990) 1.

352. J.N. Nudel, B.S. Umansky, R.O. Piagentini, and E.A. Lombardo, J. Catal. 89 (1984) 362.

353. 1. Hálasz, Appl. Catal. 47 (1989) L17.

354. B.G. Ong, C.C. Chang and D.M. Mason, Solid State Ionics, 3/4 (1981) 447.

355. B.C. Nguyen, T.A. Lin and D.A. Mason, J. Electrochem. Soc. 133 (1986) 1807.

356. S. Pancharatnam, R.A. Huggins and D.M. Mason, J. Electrochem. Soc. 122 (1975) 869.

357. C.G. Vayenas, S. Bebelis and S. Neophytides, J. Phys. Chem. 92 (1988) 5083.

358. S. Bebelis and C.G. Vayenas, J. Catal. 118 (1989) 125.

359. S. Neophytides and C.G. Vayenas, J. Catal. 118 (1989) 147.

360. C.G. Vayenas, S. Bebelis and S. Ladas, Nature 33 (1990) 625.

361. C.G. Vayenas, S. Bebelis, I.V. Yentekakis, P. Tsiakaras and H. Karasali, Platinum. Met. Rev. 34 (1990) 122.

362. E.J.L. Schouler, Solid State Ionics 9-10 (1983) 945.

363. Y. Moro-Oka, W. Ueda, S. Tanaka, and T. Ikawa, Study of Multicomponent Metal Oxide Catalysts By ${ }^{18} \mathrm{O}$ Tracer, in: Proc. 7th Int. Congr. Catal. Tokyo, 1980, Eds. T. Eiyama and K. Tanabe, Tokyo/Amsterdam, Kodansha/Elsevier, 1981, Part B, p. 1086.

364. J.F. Brazdil, D.D. Suresh, and R.K. Grasselli, J. Catal. 66 (1980) 347. 